Technical Report

MSR-TR-99-42

\title{
GIBBS STATES OF GRAPHICAL REPRESENTATIONS IN THE POTTS MODEL WITH EXTERNAL FIELDS
}

\author{
M. Biskup ${ }^{1,2,3, *}$, C. Borgs ${ }^{3}$, J.T. Chayes ${ }^{3}$ and R. Kotecký $\dot{y}^{1,3, \dagger}$ \\ ${ }^{1}$ Department of Theoretical Physics, Charles University, Prague \\ 2 Mathematisch Instituut, Katholieke Universiteit, Nijmegen \\ ${ }^{3}$ Microsoft Research, One Microsoft Way, Redmond
}

August, 1999

\begin{abstract}
We consider the ferromagnetic $q$-state Potts model, with each of the $q$ spin values coupled to an external field. We also introduce a generalized random cluster model, which includes both the Potts model in arbitrary homogeneous external fields and the non-integer $q$ random cluster model as special cases. We establish the FKG property, the finite energy condition, uniqueness of the infinite cluster, and Gibbsianness of limit states for this generalized model. Furthermore, we develop the theory of Gibbs states for the Edwards-Sokal representation of the Potts model in a field, and relate the phase structure in this representation to those in the spin and random cluster representations. Finally, we characterize the possible color(s) of the infinite cluster(s) and show that the correspondence between Edwards-Sokal Gibbs states and their random cluster marginals is bijective, once the color of the infinite cluster is fixed.
\end{abstract}

${ }^{*}$ Present Address: Microsoft Research, One Microsoft Way, Redmond

${ }^{\dagger}$ On leave from the Center for Theoretical Study, Charles University, Prague 


\section{INTRODUCTION}

In this paper, we study the ferromagnetic $q$-state Potts model with each value of the spin coupled to a distinct external field. The formal Hamiltonian of the model is

$$
H(\boldsymbol{\sigma})=-J \sum_{\langle x, y\rangle} \delta_{\sigma_{x}, \sigma_{y}}-\sum_{m=1}^{q} \sum_{x} h_{m} \delta_{\sigma_{x}, m}
$$

Here $\sigma_{x} \in\{1, \cdots, q\}$ are the spin variables, $J$ is a positive coupling constant, $\delta_{\sigma_{x}, \sigma_{y}}$ is the Kronecker delta, $\left(h_{m}\right)_{m=1}^{q}$ are real numbers representing the external fields, and $\langle x, y\rangle$ denotes a nearest-neighbor pair on $\mathbb{Z}^{d}$. The model (1.1) appears in many different contexts. For example, it arises in image processing, where $\sigma_{x}$ represents the color of the pixel labeled by $x$, and the fields $h_{m}$ lead to different a priori probabilities for different colors. Another example is a lattice gas of $q$ species, with $h_{m}$ corresponding to the fugacity of the species $m$.

During the past fifteen years, there has been a great deal of work on graphical representations of the Potts model in the absence of external fields (i.e., with $h_{m} \equiv 0$ ). In particular, the Fortuin-Kasteleyn [10] or random cluster (RC) representation has been used to prove various non-perturbative results about the Potts model using percolation-type methods (e.g., [2], [5]). In order to use the representation effectively, it was first necessary to establish certain basic features of the resulting measure, including FKG monotonicity, existence of thermodynamic limits, and properties of the Gibbs states ([2], [5], [16], [21], see also [17] and [12] for reviews).

Here we consider graphical representations of the Potts model in the presence of arbitrary external fields. This turns out to be significantly more complicated than the analysis in the absence of external fields for a number of reasons. First, when $h_{m} \equiv 0$, it is easy to verify that the RC representation has the FKG property, which is more difficult to establish here. Indeed, the FKG property does not even hold for certain boundary conditions. Second, for $h_{m} \equiv 0$, symmetry breaking in the spin representation is equivalent to percolation in the $\mathrm{RC}$ representation. Here the relationship between the phase structure of the spin model and percolation in the $\mathrm{RC}$ representation is less direct; in some cases the percolation threshold corresponds to no phase transition at all in the spin model [3]. Third, absence of symmetry raises the question of the color(s) of the infinite cluster(s), a question which turns out to be quite intricate, and does not need to be addressed for $h_{m} \equiv 0$.

Our work was motivated by an attempt to understand the phase diagram of the model (1.1), using both cluster expansion and percolation techniques; our results on the phase diagram are presented in a separate paper [3]. In this paper we generalize known results on the properties of Gibbs states of the RC models to systems with external fields. In particular, for the RC model in an arbitrary homogeneous magnetic field, we prove FKG properties, existence of infinite volume measures, and that these measures are Gibbs states. See also [3] and [4] for other graphical representations of Potts models in an external field.

In addition, we develop the theory of Gibbs states for the so-called Edwards-Sokal (ES) measure, a measure on both spin and bond variables which was originally introduced in order to explain the Swendsen-Wang algorithm for sampling from the Potts model [8]. In a finite volume, the marginals of the ES measure are just the spin and the RC measures. Here we consider infinite volume ES measures as interesting and important probabilistic objects in their own right. In particular, we introduce the notion of ES Gibbs measures, and analyze whether (or under what conditions) the marginals of such Gibbs measures are Gibbs measures of the 
corresponding spin and random cluster models. We clarify this relationship, and in the process derive properties of the spaces of Gibbs states for all three representations.

We believe that the rigorous analysis of properties of the Potts model in terms of the ES representation will prove to be quite fruitful in future work. Indeed, while the ES representation shares many of the more useful properties of the random cluster representation, it does not share all of its difficulties. In particular, the ES representation is quasilocal, ${ }^{1}$ while the RC representation is not. Much of the standard theory of Gibbs states (as well as its physical interpretation) requires quasilocality ([15], [20], [22]). Absence of this property has been a major technical impediment in the analysis of Gibbs states for the RC representation.

Finally, we consider the question of RC models in a field with non-integer values of $q$. Although the spin representation of the Potts model (and therefore also the Edwards-Sokal representation) only admits an integer number of spin states, it has been realized for some time that the standard RC measure in the absence of a field is perfectly well-defined for non-integer values of $q$. Provided that $q \geq 1$, the resulting finite volume measures with free and wired boundary conditions are FKG, which allows one to prove the existence of the corresponding infinite volume measures. However, the most straightforward version of the $\mathrm{RC}$ model in a field reduces to a model with integer $q$ when we take $h_{m} \equiv 0$. Explicitly, the RC model in a field defined on bond configurations $\boldsymbol{\eta}=\left\{\eta_{\langle x, y\rangle}\right\}, \eta_{\langle x, y\rangle} \in\{0,1\}$, has weights of the form

$$
\prod_{\langle x, y\rangle: \eta_{\langle x, y\rangle}=1}\left(e^{\beta J}-1\right) \delta_{\sigma_{x}, \sigma_{y}} \prod_{C} \Theta(C),
$$

where the second product is over all connected components of sites, and the weights of the components are given by

$$
\Theta(C)=\sum_{m=1}^{q} e^{h_{m}|\mathbb{V}(C)|} .
$$

Here $|\mathbb{V}(C)|$ denotes the volume of the cluster $C$. Notice that when $h_{m} \equiv 0$, the weights $(1.2)$ reduce to the more familiar weights ${ }^{2}$

$$
\left(e^{\beta J}-1\right)^{n(\boldsymbol{\eta})} q^{c(\boldsymbol{\eta})},
$$

where $n(\boldsymbol{\eta})$ is the number of bonds $\langle x, y\rangle$ with $\eta_{\langle x, y\rangle}=1$ in configuration $\boldsymbol{\eta}$, and $c(\boldsymbol{\eta})$ is the number of connected components of sites in $\boldsymbol{\eta}$.

Thus we also propose a generalized random cluster (GRC) model with the weight $\Theta(c)$ in (1.2) replaced by

$$
\widetilde{\Theta}(C)=\sum_{m=1}^{q} q_{m} e^{h_{m}|\mathbb{V}(C)|},
$$

\footnotetext{
${ }^{1}$ Recall that quasilocality is the property of continuity (in the product topology) of finite volume Gibbs states with respect to boundary conditions.

${ }^{2}$ Even the weights (1.4) may not be entirely familiar to readers who know the RC weights as (1$\left.e^{-\beta J}\right)^{n(\boldsymbol{\eta})}\left(e^{-\beta J}\right)^{v(\boldsymbol{\eta})} q^{c(\boldsymbol{\eta})}$, where $v(\boldsymbol{\eta})$ is the number of bonds $\langle x, y\rangle$ with $\eta_{\langle x, y\rangle}=0$ in configuration $\boldsymbol{\eta}$. The only difference between the latter weights and (1.4) is an overall normalization factor, which makes no difference in the resulting measure.
} 
where the $q_{m}$ are non-integer parameters. Provided that the $q_{m}$ are positive and satisfy the condition

$$
\sum_{m: h_{m}=h_{\max }} q_{m} \geq 1
$$

where $h_{\max }$ is the maximum value of the component fields $h_{m}$, we will be able to prove that resulting finite volume measures with certain boundary conditions are FKG, and hence that the corresponding infinite volume measures exist. We expect that many of our other results for the RC model in a field hold also for this generalized model, but we have not explicitly verified this.

Notice the following two special cases of the generalized model with the weights (1.5). If we take $q_{m}=1, m=1, \cdots, q$, then we get (1.3), i.e., the random cluster representation of the Potts model in an external field. On the other hand, if we take $h_{m}=0$ for all $m$, we get the weights (1.4) with $q$ replaced by $\sum_{m} q_{m}$, which is in general non-integer. Thus the GRC model generalizes both the non-vanishing external field case and the standard non-integer $q$ model.

It turns out that the set of "colors" $m \in\{1, \cdots, q\}$ with $h_{m}=h_{\max }$ will play an important role in the analysis of both of the above described random cluster models in a field. In the standard model (with $h_{m} \equiv 0$ ), it is well-known that the extremal measures are obtained by applying free and "wired" boundary conditions. The latter are the marginals of measures in which all spins on the boundary are set to a fixed color $m \in\{1, \cdots, q\}$, and thus identified as one component in the $\mathrm{RC}$ representation. In this work, we will find that the extremal measures are obtained by applying free and what we call "maxwired" boundary conditions. Measures with maxwired boundary conditions are the marginals of measures in which all spins on the boundary are set a color $m$ for which $h_{m}=h_{\max }$. The other RC wired measures, i.e. those with boundary conditions set to a color $m$ for which $h_{m}<h_{\max }$, are hard to analyze because they do not even obey the FKG inequality.

We end this section with a summary of our results:

In Section 2, we state our theorems on mappings between the sets of ES Gibbs states and spin and RC Gibbs states, respectively. In particular, Theorem 2.1 implies that the relevant marginals of the infinite volume ES Gibbs states are spin Gibbs states. The same is not true for the RC states unless we restrict to states with no more than one infinite cluster, as we do in Theorem 2.2. We also formulate results (Theorems 2.3 and 2.4) on the existence of infinite volume measures for the RC and ES representations with free and maxwired boundary conditions. Finally, we state a result (Theorem 2.5) relating uniqueness or non-uniqueness of Gibbs states to the absence or presence of infinite clusters. In two dimensions, we are able to prove more - namely that, away from the transition temperature, the RC Gibbs state is unique, and similarly for the ES state, provided there is only one color $m$ with $h_{m}=h_{\max }$ (Theorem 2.6).

In Section 3, we introduce the generalized random cluster (GRC) model and formulate its FKG monotonicity properties. In particular, Theorem 3.1 states that the free and maxwired GRC states are strong FKG, and hence that the corresponding infinite volume limits exists. This theorem also asserts that, in the FKG order, every GRC Gibbs state lies between these two infinite volume states. Finally, this theorem compares GRC states at different couplings and different sets of external fields (with an appropriately defined partial order). As a corollary, we prove various properties of the relevant percolation probabilities, which are the order parameters for the transition. Theorem 3.2 deals with RC marginals of ES Gibbs states. In particular, it states that the infinite volume RC maxwired measure dominates all such marginals, 
M. BISKUP, C. BORGS, J.T. CHAYES, R. KOTECKÝ

while the free RC measure is dominated by the marginals of all ES Gibbs states with at most one infinite cluster. Our final results establish uniqueness of the infinite cluster for translation invariant GRC Gibbs and limit states (Theorem 3.3), and give a stronger version of the DLR equation for any GRC Gibbs state with a unique infinite cluster. (Theorem 3.4).

Our results are proved in Sections 4-10. In Sections 4-6 we prove the theorems stated in Section 3 (in the order of their appearance). The theorems of Section 2 are proven in the remaining Sections 7-10 (in the order 2.4, 2.5, 2.6, 2.1 and 2.2). Theorem 2.3, which is an easy corollary of the results of Section 3, is proved at the end of Section 6 .

\section{Gibbs States in the Edwards-Sokal, Spin and Random Cluster REPRESENTATIONS}

In this section we define Gibbs measures for joint probability spaces of spin and bond variables, i.e., the Edwards-Sokal Gibbs measures. We then relate the set of Edwards-Sokal Gibbs measures to the more standard sets of spin and random cluster Gibbs measures.

We begin with some notation. For any subset $\Lambda \subset \mathbb{Z}^{d}$, we introduce $\mathbb{B}_{0}(\Lambda)$ as the set of all bonds $b=\langle x, y\rangle$ of nearest neighbors with both endpoints in $\Lambda$ and $\mathbb{B}(\Lambda)$ as the set of all bonds with at least one endpoint in $\Lambda$. For any $\mathbb{B} \subset \mathbb{B}_{0}\left(\mathbb{Z}^{d}\right)$, we define $\mathbb{V}(\mathbb{B})$ as the set of sites which belong to at least one bond in $\mathbb{B}$.

To motivate our definitions, we first derive the Edward-Sokal representation for a finite box $\Lambda \subset \mathbb{Z}^{d}$ with free boundary conditions. For free boundary conditions, the Gibbs factor of the $q$-state Potts model in a general field is given by

$$
e^{-\beta H\left(\boldsymbol{\sigma}_{\Lambda}\right)}=\prod_{\langle x, y\rangle \in \mathbb{B}_{0}(\Lambda)} e^{\beta J \delta_{\sigma_{x}, \sigma_{y}}} \prod_{x \in \Lambda} e^{\beta h\left(\sigma_{x}\right)},
$$

where $\left(h_{m}\right)_{m=1}^{q} \in \mathbb{R}^{q}$ is a collection of arbitrary fields and $h\left(\sigma_{x}\right)$ stands for

$$
h\left(\sigma_{x}\right)=\sum_{m=1}^{q} h_{m} \delta_{\sigma_{x}, m} .
$$

In order to derive the Edwards-Sokal (ES) and random cluster (RC) representation, we rewrite the Gibbs factor by expanding each term $e^{\beta J \delta_{\sigma_{x}, \sigma_{y}}}$ as $1+\left(e^{\beta J}-1\right) \delta_{\sigma_{x}, \sigma_{y}}$. Introducing bond configurations $\boldsymbol{\eta}_{\mathbb{B}_{0}(\Lambda)}=\left\{\eta_{b}\right\}_{b \in \mathbb{B}_{0}(\Lambda)}$ with $\eta_{b} \in\{0,1\}$, we can write the Gibbs factor (2.1) as the sum

$$
e^{-\beta H\left(\boldsymbol{\sigma}_{\Lambda}\right)}=\sum_{\boldsymbol{\eta}_{\mathbb{B}_{0}(\Lambda)}} \prod_{\substack{b=\langle x, y\rangle \in \mathbb{B}_{0}(\Lambda) \\ \eta_{\langle x, y\rangle}=1}}\left(e^{\beta J}-1\right) \delta_{\sigma_{x}, \sigma_{y}} \prod_{x \in \Lambda} e^{\beta h\left(\sigma_{x}\right)} .
$$

The key point of this reformulation is that $\boldsymbol{\eta}$ can now be treated in the same way as $\boldsymbol{\sigma}$; one just peels off the first sum in (2.3) and interprets the remainder as a joint weight of $\boldsymbol{\sigma}$ and $\boldsymbol{\eta}$. In this manner one obtains the finite volume Gibbs measure of the Potts model as the spin marginal of a measure on both spin and bond configurations - the Edwards-Sokal measure. The bond configuration marginal is then the random cluster measure.

So far we have considered only free boundary conditions. Instead of modifying the preceding argument for other boundary conditions, we directly introduce the notion of infinite volume Gibbs measures on the joint space of spin and bond variables. To define the Gibbs ES states, let us introduce for any pair of (not necessarily related) finite sets $\Lambda \subset \mathbb{Z}^{d}, \mathbb{B} \subset \mathbb{B}_{0}\left(\mathbb{Z}^{d}\right)$, and 
any fixed configurations $\boldsymbol{\sigma}_{\Lambda^{\mathrm{c}}}, \boldsymbol{\eta}_{\mathbb{B}^{\mathrm{c}}}$ outside of them, the measure $\mu_{\Lambda, \mathbb{B}}^{\mathrm{ES}}\left(\cdot \mid \boldsymbol{\sigma}_{\Lambda^{\mathrm{c}}}, \boldsymbol{\eta}_{\mathbb{B}^{\mathrm{B}}}\right)$ by

$$
\mu_{\Lambda, \mathbb{B}}^{\mathrm{ES}}\left(\boldsymbol{\sigma}_{\Lambda}, \boldsymbol{\eta}_{\mathbb{B}} \mid \boldsymbol{\sigma}_{\Lambda^{\mathrm{c}}}, \boldsymbol{\eta}_{\mathbb{B}^{\mathrm{c}}}\right)=\frac{W\left(\boldsymbol{\sigma}_{\Lambda}, \boldsymbol{\eta}_{\mathbb{B}} \mid \boldsymbol{\sigma}_{\Lambda^{\mathrm{c}}}, \boldsymbol{\eta}_{\mathbb{B}^{\mathrm{c}}}\right)}{\sum_{\overline{\boldsymbol{\sigma}}_{\Lambda}, \overline{\boldsymbol{\eta}}_{\mathbb{B}}} W\left(\overline{\boldsymbol{\sigma}}_{\Lambda}, \overline{\boldsymbol{\eta}}_{\mathbb{B}} \mid \boldsymbol{\sigma}_{\Lambda^{\mathrm{c}}}, \boldsymbol{\eta}_{\mathbb{B}^{\mathrm{c}}}\right)},
$$

where the convention ${ }^{3} \mu_{\Lambda, \mathbb{B}}^{\mathrm{ES}}\left(\boldsymbol{\sigma}_{\Lambda}, \boldsymbol{\eta}_{\mathbb{B}} \mid \boldsymbol{\sigma}_{\Lambda^{\mathrm{c}}}, \boldsymbol{\eta}_{\mathbb{B}^{\mathrm{c}}}\right)=0$ is assumed for the case that the sum in the denominator vanishes, and where

$$
W\left(\boldsymbol{\sigma}_{\Lambda}, \boldsymbol{\eta}_{\mathbb{B}} \mid \boldsymbol{\sigma}_{\Lambda^{\mathrm{c}}}, \boldsymbol{\eta}_{\mathbb{B}^{\mathrm{c}}}\right)=\prod_{\substack{\langle x, y\rangle \in \mathbb{B} \cup \mathbb{B}(\Lambda) \\ \eta_{\langle x, y\rangle}=1}}\left(e^{\beta J}-1\right) \delta_{\sigma_{x}, \sigma_{y}} \prod_{x \in \Lambda} e^{\beta h\left(\sigma_{x}\right)} .
$$

The dependence on parameters $J$ and $\left\{h_{m}\right\}$ will be explicitly marked only when a reference to them is needed.

Our first theorem concerns the relation between the ES and spin Gibbs measures. Let $\mathcal{G}^{\mathrm{ES}}$ be the set of all infinite volume Gibbs ES states defined by imposing the DLR equations with specification (2.4). Namely, $\nu \in \mathcal{G}^{\mathrm{ES}}$ iff

$$
\nu(f)=\int \nu(\mathrm{d} \boldsymbol{\sigma}, \mathrm{d} \boldsymbol{\eta}) \mu_{\Lambda, \mathbb{B}}^{\mathrm{ES}}\left(f \mid \boldsymbol{\sigma}_{\Lambda^{\mathrm{c}}}, \boldsymbol{\eta}_{\mathbb{B}^{\mathrm{c}}}\right)
$$

for all pairs of finite sets $\Lambda$ and $\mathbb{B}$ and any cylinder function $f$ depending only on $\boldsymbol{\sigma}_{\Lambda}$ and $\boldsymbol{\eta}_{\mathbb{B}}$. Note that the fact that the underlying "set of sites" contains both the set $\mathbb{Z}^{d}$ and the set $\mathbb{B}_{0}\left(\mathbb{Z}^{d}\right)$ does not prevent the abstract theory of Gibbs states - in the version that allows for "hard-core interactions" (c.f., [22], [20], [15]) — from being applied. The important property, quasilocality of the specification $\left\{\mu_{\Lambda, \mathbb{B}}^{\mathrm{ES}}\right\}$, is clearly satisfied, implying, in particular, that the set of Gibbs states $\mathcal{G}^{\mathrm{ES}}$ is not empty. Note also that quasilocality and consistency of the specifications imply that the DLR condition (2.6) is equivalent to the (apparently stronger) statement that the conditional expectations of $\nu$ are given by (2.4), i.e.

$$
\nu\left(f \mid \boldsymbol{\sigma}_{\Lambda^{\mathrm{c}}}, \boldsymbol{\eta}_{\mathbb{B}^{\mathrm{c}}}\right)=\mu_{\Lambda, \mathbb{B}}^{\mathrm{ES}}\left(f \mid \boldsymbol{\sigma}_{\Lambda^{\mathrm{c}}}, \boldsymbol{\eta}_{\mathbb{B}^{\mathrm{c}}}\right) \quad \nu \text {-a.s. }
$$

for all pairs of finite sets $\Lambda$ and $\mathbb{B}$ and any cylinder function $f$ depending only on $\boldsymbol{\sigma}_{\Lambda}$ and $\boldsymbol{\eta}_{\mathbb{B}}$.

Let $\mathcal{G}^{\text {SPIN }}$ denote the set of all spin Gibbs states, defined by means of the DLR condition and the Hamiltonian (1.1), appropriately modified to incorporate the boundary condition. Let $\Pi_{S}$ denote the mapping that assigns the spin marginal to any infinite volume ES measure. It is not a priori obvious that the spin marginal of any infinite volume Gibbs ES state is an infinite volume Gibbs spin state. However, it turns out that even a little more is true.

Theorem 2.1 The mapping $\Pi_{\mathrm{S}}$ is a linear isomorphism between the Choquet simplices ${ }^{4} \mathcal{G}^{\mathrm{ES}}$ and $\mathcal{G}^{\mathrm{SPIN}}$. When restricted to translation invariant measures, $\Pi_{\mathrm{S}}$ is an isomorphism between the simplex of all translation invariant Gibbs ES states and the simplex of all translation invariant Gibbs spin states. In particular, $\left|\mathcal{G}^{\mathrm{ES}}\right|=1$ if and only if $\left|\mathcal{G}^{\mathrm{spIN}}\right|=1$.

Remark. The last statement is false for the correspondence between ES Gibbs states and their RC marginals. For instance, for $d=2$ it is known that there are exactly two extremal Ising Gibbs states below the critical temperature ([11], [1], [18]) and, therefore, two extremal ES Gibbs states, while the corresponding RC marginals are identical.

\footnotetext{
${ }^{3}$ Here we use the theory of Gibbs states as presented by Ruelle [22], who explicitly considers models with configuration spaces determined by local restriction rules (hard cores). See Sections 1.1 and 1.5 of [22].

${ }^{4}$ See, e.g., [22], [15] and [20] for the definition of Choquet simplices.
} 
As alluded to in the introduction, RC Gibbs measures have finite volume specifications that are not quasilocal, which prevents the straightforward application of the general theory of Gibbs states. It therefore is often more convenient to consider ES Gibbs measures, whose finite volume specifications are local, and study RC measures only as their marginals. The relation of these marginals to RC Gibbs measures as introduced in [9], [16], [21], and [5] for Potts models without magnetic fields is the content of our next theorem.

First, however, we generalize the notion of RC Gibbs states to Potts models with magnetic fields. To this end we introduce, for any configuration $\boldsymbol{\eta}$ on $\mathbb{B}_{0}\left(\mathbb{Z}^{d}\right)$, the set of occupied bonds $\mathbb{B}_{\text {occ }}(\boldsymbol{\eta})=\left\{b \in \mathbb{B}_{0}\left(\mathbb{Z}^{d}\right): \eta_{b}=1\right\}$ and the corresponding graph $\left(\mathbb{Z}^{d}, \mathbb{B}_{\text {occ }}(\boldsymbol{\eta})\right)$ with the vertex set $\mathbb{Z}^{d}$ and the edge set $\mathbb{B}_{\text {occ }}(\boldsymbol{\eta})$. For any connected component $C(\boldsymbol{\eta})$ of this graph (possibly a single site), we use $\mathbb{V}(C(\boldsymbol{\eta}))$ to denote the corresponding vertex set. We now define, for any finite set of bonds $\mathbb{B}$ and any configuration $\boldsymbol{\eta}_{\mathbb{B}^{c}}$, the measure

$$
\mu_{\mathbb{B}}^{\mathrm{RC}}\left(\boldsymbol{\eta}_{\mathbb{B}} \mid \boldsymbol{\eta}_{\mathbb{B}^{c}}\right)=\frac{W_{\mathbb{B}}^{\mathrm{RC}}\left(\boldsymbol{\eta}_{\mathbb{B}} \mid \boldsymbol{\eta}_{\mathbb{B}^{c}}\right)}{\sum_{\overline{\boldsymbol{\eta}}_{\mathbb{B}}} W_{\mathbb{B}}^{\mathrm{RC}}\left(\overline{\boldsymbol{\eta}}_{\mathbb{B}} \mid \boldsymbol{\eta}_{\mathbb{B}^{c}}\right)}
$$

with

$$
W_{\mathbb{B}}^{\mathrm{RC}}\left(\boldsymbol{\eta}_{\mathbb{B}} \mid \boldsymbol{\eta}_{\mathbb{B}^{\mathrm{c}}}\right)=\left(e^{\beta J}-1\right)^{\left|\mathbb{B}_{\mathrm{occ}}(\boldsymbol{\eta}) \cap \mathbb{B}\right|} \prod_{C(\boldsymbol{\eta}): \mathbb{V}(C(\boldsymbol{\eta})) \cap \mathbb{V}(\mathbb{B}) \neq \emptyset} \sum_{m=1}^{q} e^{-\beta\left(h_{\max }-h_{m}\right)|\mathbb{V}(C(\boldsymbol{\eta}))|},
$$

where the product runs over all connected components $C(\boldsymbol{\eta})$ such that the vertex set $\mathbb{V}(C(\boldsymbol{\eta}))$ intersects the set $\mathbb{V}(\mathbb{B})$, and $h_{\max }$ is used to denote

$$
h_{\max }=\max _{m \in\{1, \ldots, q\}} h_{m} .
$$

Interpreting $e^{-\infty}=0$, any infinite cluster $C(\boldsymbol{\eta})$ intersecting $\mathbb{V}(\mathbb{B})$ contributes just the factor $q_{0}=\left|Q_{\max }(h)\right|$, the size of the set

$$
Q_{\max }(h)=\left\{m \in\{1, \ldots, q\} \mid h_{m}=h_{\max }\right\} .
$$

For future reference, we also define $N_{\infty}=N_{\infty}(\boldsymbol{\eta})$ as the random variable denoting the number of infinite clusters of $\mathbb{B}_{\text {occ }}(\boldsymbol{\eta})$, and use $C_{\infty}=C_{\infty}(\boldsymbol{\eta})$ to denote the unique infinite cluster whenever $N_{\infty}=1$.

As usual, one introduces the set of Gibbs states $\mathcal{G}^{\mathrm{RC}}$ as the set of measures $\mu$ on $\{0,1\}^{\mathbb{B}_{0}\left(\mathbb{Z}^{d}\right)}$ that satisfy the DLR equation

$$
\mu(f)=\int \mu(\mathrm{d} \boldsymbol{\eta}) \mu_{\mathbb{B}}^{\mathrm{RC}}\left(f \mid \boldsymbol{\eta}_{\mathbb{B}^{c}}\right)
$$

for any finite $\mathbb{B}$ and any cylinder function $f$ with support in $\mathbb{B}$. Note that, in contrast to equations (2.6) and (2.7), here the DLR condition (2.12) does not imply that the conditional expectations of an RC Gibbs state $\mu$ are given by the finite volume expectations (2.8) due to the lack of quasilocality. However, it turns out that uniqueness of the infinite cluster is enough to ensure that the DLR condition implies a statement of the form (2.7) (see Theorem 3.4).

As already observed in [5], the above notion of RC Gibbs states does not accommodate all "naturally arising" limiting states. When reformulated in terms of the ES measures, not every $\mathrm{RC}$ marginal of an ES Gibbs measure is an RC Gibbs state. An example is the ES Gibbs state corresponding to the standard Dobrushin state with a stable interface between two ordered states.

However, when restricted to the set of ES measures with at most one infinite cluster, the situation changes. As it turns out, not only is the marginal of every such ES Gibbs measure an 
GIBBS STATES OF GRAPHICAL REPRESENTATIONS IN THE POTTS MODEL

RC Gibbs measure, but also each RC Gibbs measure with at most one infinite cluster can be obtained as a marginal of a suitable ES Gibbs measure. In addition, a natural refinement holds: up to a choice of the "color" of the infinite cluster, the surjective correspondence between ES and RC measures is actually one-to-one.

To state the next theorem, we use $\mathcal{G}_{<1}^{\mathrm{ES}}=\left\{\nu \in \mathcal{G}^{\mathrm{ES}} \mid \nu\left(N_{\infty} \leq 1\right)=1\right\}$ to denote the set of ES Gibbs measures such that with probability one there is at most one infinite cluster of occupied bonds. Similarly, let $\mathcal{G}_{\leq 1}^{\mathrm{RC}}=\left\{\mu \in \mathcal{G}^{\mathrm{RC}} \mid \mu\left(N_{\infty} \leq 1\right)=1\right\}, \mathcal{G}_{k}^{\mathrm{ES}}=\left\{\nu \in \mathcal{G}^{\mathrm{ES}} \mid \nu\left(N_{\infty}=k\right)=1\right\}$ and $\mathcal{G}_{k}^{\mathrm{RC}}=\left\{\mu \in \mathcal{G}^{\mathrm{RC}} \mid \mu\left(N_{\infty}=k\right)=1\right\}, k=0,1$. Also let $\mathcal{G}_{1, m}^{\mathrm{ES}}=\left\{\nu \in \mathcal{G}^{\mathrm{ES}} \mid \nu\left(\mathcal{A}_{1, m}^{\infty}\right)=1\right\}$, where $\mathcal{A}_{1, m}^{\infty}$ is the event $\mathcal{A}_{1, m}^{\infty}=\left\{N_{\infty}=1\right.$ and $\sigma_{x}=m$ for all $\left.x \in \mathbb{V}\left(C_{\infty}\right)\right\}$. Finally, let $\Pi_{\mathrm{RC}}$ be the mapping that assigns RC marginals to ES Gibbs measures.

Theorem 2.2 (i) The restriction of the map $\Pi_{\mathrm{RC}}$ to $\mathcal{G}_{\leq 1}^{\mathrm{ES}}$ is surjective onto $\mathcal{G}_{\leq 1}^{\mathrm{RC}}$.

(ii) Every $\nu \in \mathcal{G}_{\leq 1}^{\mathrm{ES}}$ has a unique decomposition

$$
\nu=\lambda_{0} \nu_{0}+\sum_{m \in Q_{\max }} \lambda_{m} \nu_{m}
$$

with $\nu_{0} \in \mathcal{G}_{0}^{\mathrm{ES}}, \nu_{m} \in \mathcal{G}_{1, m}^{\mathrm{ES}}, \lambda_{0}, \lambda_{m} \geq 0$, and $\lambda_{0}+\sum_{m \in Q_{\max }} \lambda_{m}=1$.

(iii) The restriction of the map $\Pi_{\mathrm{RC}}$ to $\mathcal{G}_{0}^{\mathrm{ES}}$ is one-to-one from $\mathcal{G}_{0}^{\mathrm{ES}}$ to $\mathcal{G}_{0}^{\mathrm{RC}}$. If $m \in Q_{\max }(h)$, then the restriction of $\Pi_{\mathrm{RC}}$ to $\mathcal{G}_{1, m}^{\mathrm{ES}}$ is one-to-one from $\mathcal{G}_{1, m}^{\mathrm{ES}}$ to $\mathcal{G}_{1}^{\mathrm{RC}}$.

(iv) If $\left|Q_{\max }(h)\right|=1$, then the mapping $\Pi_{\mathrm{RC}}$ is a bijection $\Pi_{\mathrm{RC}}: \mathcal{G}_{\leq 1}^{\mathrm{ES}} \rightarrow \mathcal{G}_{\leq 1}^{\mathrm{RC}}$.

Remarks. (i) As we will see in the next section, the set $\mathcal{G}_{\leq 1}^{\mathrm{ES}}$ is non-empty. By the above theorem, this implies that also $\mathcal{G}_{\leq 1}^{\mathrm{RC}}$ is non-empty.

(ii) Since $\left\{N_{\infty}=0\right\}$ is a tail event, it follows from the standard theory of Gibbs states and the fact that the specifications (2.4) of the Edwards-Sokal measure are quasilocal, that the conditional measure $\nu\left(\cdot \mid N_{\infty}=k\right), k=0,1$, is a Gibbs state for any $\nu \in \mathcal{G}_{\leq 1}^{\text {ES }}$ with $0<$ $\nu\left(N_{\infty}=0\right)<1$. Although the corresponding statement is not known a priori for a $\mathrm{RC}$ Gibbs state $\mu \in \mathcal{G}_{\leq 1}^{\mathrm{RC}}$ (due to lack of quasilocality), it is a consequence of statement (i) and the commutativity of the following diagram,

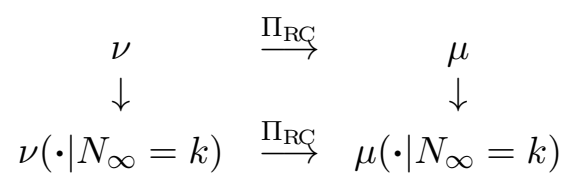

which, in turn, is a consequence of Theorem 2.2(ii).

Next, we state our results on the existence of thermodynamic limits for the extremal ES and RC Gibbs measures with free and wired boundary conditions. We begin by introducing the relevant finite volume ES measures. Observing that, for a finite volume $\Lambda$, the state $\mu_{\Lambda, \mathbb{B}(\Lambda)}^{\mathrm{ES}}\left(\cdot \mid \boldsymbol{\sigma}_{\Lambda^{\mathrm{c}}}, \boldsymbol{\eta}_{\mathbb{B}(\Lambda)^{\mathrm{c}}}\right)$ does not depend on $\boldsymbol{\eta}_{\mathbb{B}(\Lambda)^{\mathrm{c}}}$, we define the measure

$$
\mu_{\Lambda, m}^{\mathrm{ES}}(\cdot)=\mu_{\Lambda, \mathbb{B}(\Lambda)}^{\mathrm{ES}}\left(\cdot \mid \boldsymbol{\sigma}_{\Lambda^{\mathrm{c}}}^{m}, \boldsymbol{\eta}_{\mathbb{B}(\Lambda)^{\mathrm{c}}}\right),
$$

where $\boldsymbol{\sigma}^{m}$ is the constant configuration, $\sigma_{x}^{m}=m$ for all $x \in \mathbb{Z}^{d}$, with $m \in\{1, \ldots, q\}$. In a similar way, the measure $\mu_{\Lambda, \mathbb{B}_{0}(\Lambda)}^{\mathrm{ES}}\left(\cdot \mid \boldsymbol{\sigma}_{\Lambda^{\mathrm{c}}}, \boldsymbol{\eta}_{\mathbb{B}_{0}(\Lambda)^{\mathrm{c}}}\right)$ does not depend on $\boldsymbol{\sigma}_{\Lambda^{\mathrm{c}}}$, provided that the $\boldsymbol{\eta}$-boundary condition is chosen as $\boldsymbol{\eta}_{\mathbb{B}_{0}(\Lambda)^{\mathrm{c}}}=\boldsymbol{\eta}_{\mathbb{B}_{0}(\Lambda) \mathrm{c}}^{0}$, where $\boldsymbol{\eta}^{0}$ denotes the configuration with 
$\eta_{b}^{0}=0$ for all $b \in \mathbb{B}\left(\mathbb{Z}^{d}\right)$. In this case we introduce the measure

$$
\mu_{\Lambda, \text { free }}^{\mathrm{ES}}(\cdot)=\mu_{\Lambda, \mathbb{B}_{0}(\Lambda)}^{\mathrm{ES}}\left(\cdot \mid \boldsymbol{\sigma}_{\Lambda^{\mathrm{c}}}, \boldsymbol{\eta}_{\mathbb{B}_{0}(\Lambda)^{\mathrm{c}}}^{0}\right) .
$$

The $\boldsymbol{\eta}$-marginals of the measures $\mu_{\Lambda, \text { free }}^{\mathrm{ES}}(\cdot)$ and $\mu_{\Lambda, m}^{\mathrm{ES}}$ are the $\mathrm{RC}$ measures $\mu_{\Lambda, \text { free }}^{\mathrm{RC}}(\cdot)$ and $\mu_{\Lambda, m}^{\mathrm{RC}}$ with free and $m$-wired boundary conditions, respectively. A particular role will be played by the RC measures with $m$-wired boundary conditions such that $m \in Q_{\max }(h)$, i.e., $h_{m}=h_{\max }$. Note that the measures $\mu_{\Lambda, m}^{\mathrm{RC}}$ are identical for all values $m \in Q_{\max }(h)$; we will use $\mu_{\Lambda \text {,maxwir }}^{\mathrm{RC}}$ to denote any of them.

Theorem 2.3 Let $\beta \geq 0, J \geq 0$, and $h_{m} \in \mathbb{R}, m=1, \ldots, q$.

(i) Let $f$ be a quasilocal function on $\{0,1\}^{\mathbb{B}_{0}\left(\mathbb{Z}^{d}\right)}$. Then the limits

$$
\mu_{\operatorname{maxwir}}^{\mathrm{RC}}(f)=\lim _{\Lambda \nearrow \mathbb{Z}^{d}} \mu_{\Lambda, \text { maxwir }}^{\mathrm{RC}}(f)
$$

and

$$
\mu_{\text {free }}^{\mathrm{RC}}(f)=\lim _{\Lambda \nearrow \mathbb{Z}^{d}} \mu_{\Lambda, \text { free }}^{\mathrm{RC}}(f)
$$

exist and are translation invariant.

(ii) The measures $\mu_{\mathrm{maxwir}}^{\mathrm{RC}}$ and $\mu_{\text {free }}^{\mathrm{RC}}$ are $R C$ Gibbs states with at most one infinite cluster.

Remarks. (i) The limit $\Lambda \nearrow \mathbb{Z}^{d}$ above (and hereafter) is taken in the sense of the limit along the net $\left\{\Lambda \subset \mathbb{Z}^{d}\right.$ finite $\}$ with the net ordering given by the set inclusion. However, when we talk about a general RC limit state, we will have a weaker notion in mind. Namely, we say that a measure $\mu$ on $\{0,1\}^{\mathbb{B}_{0}\left(\mathbb{Z}^{d}\right)}$ is an RC limit state if there is a sequence of finite sets $\mathbb{B}_{n} \subset \mathbb{B}_{0}\left(\mathbb{Z}^{d}\right)$ and a sequence of configurations $\boldsymbol{\eta}^{(n)}$ such that $\mu(f)=\lim _{n \rightarrow \infty} \mu_{\mathbb{B}_{n}}^{\mathrm{RC}}\left(f \mid \boldsymbol{\eta}_{\mathbb{B}_{n}}^{(n)}\right)$.

(ii) We will prove the existence of the limit (2.17) by first establishing that the $\mu_{\Lambda, m}^{\mathrm{RC}}$ is strong FKG if $h_{m}=h_{\max }$, see Theorem 3.1. The requirement $h_{m}=h_{\max }$ is crucial for our proof of Theorem 3.1, since the proof relies on the FKG property of the finite volume measures $\mu_{\Lambda, m}^{\mathrm{RC}}$. In fact, for $\beta$ large enough, a contour argument indicates that $\mu_{\Lambda, m}^{\mathrm{RC}}$ with $h_{m}<h_{\max }$ is not even FKG.

(iii) The statements of Theorem 2.3 are special cases of those of Theorem 3.1 (ii), Theorem 3.3 and its corollary, which hold for the GRC models discussed in the introduction.

By using the general theorem on the uniqueness of the infinite cluster [6], the conclusion about the existence of the limiting RC measures can be strengthened to their ES preimages:

Theorem 2.4 Let $\beta \geq 0$ and $h_{m} \in \mathbb{R}, m=1, \ldots, q$. If $m \in Q_{\max }(h)$, then the weak limits

$$
\mu_{m}^{\mathrm{ES}}=\lim _{\Lambda \nearrow \mathbb{Z}^{d}} \mu_{\Lambda, m}^{\mathrm{ES}}
$$

and

$$
\mu_{\text {free }}^{\mathrm{ES}}=\lim _{\Lambda \nearrow \mathbb{Z}^{d}} \mu_{\Lambda, \text { free }}^{\mathrm{ES}}
$$

exist and are translation invariant ES Gibbs states with at most one infinite cluster.

Remark. In contrast to Theorem 2.3, the statement here that the limiting measures are Gibbs states is a trivial consequence of the general theory of Gibbs states for systems with quasilocal interactions. 
Next, we state a theorem relating the uniqueness or non-uniqueness of Gibbs states to the existence of an infinite cluster. To this end, we define the percolation probability

$$
P_{\infty}(\beta, J, h)=\sup _{\mu \in \widetilde{\mathcal{G}}^{\mathrm{RC}}} \mu\left(\left|C_{0}\right|=\infty\right)
$$

and the auxiliary percolation probability

$$
\widetilde{P}_{\infty}(\beta, J, h)=\inf _{\mu \in \widetilde{\mathcal{G}}^{\mathrm{RC}}} \mu\left(\left|C_{0}\right|=\infty\right) .
$$

where $C_{0}=C_{0}(\boldsymbol{\eta})$ is the cluster that contains the origin $0 \in \mathbb{Z}^{d}$, and where we have restricted ourselves to the set $\widetilde{\mathcal{G}}^{\mathrm{RC}}$ of all translation invariant RC Gibbs measures. As we will see in the next section (corollary to Theorem 3.1), the density $P_{\infty}(\beta, J, h)$ is just the probability of percolation in the measure $\mu_{\text {maxwir }}^{\mathrm{RC}}$, and is a nondecreasing, right continuous function of $J$. Similarly, $\widetilde{P}_{\infty}(\beta, J, h)$ is the probability of percolation in the measure $\mu_{\text {free }}^{\mathrm{RC}}$. We also define the critical coupling,

$$
J_{c}(\beta, h)=\inf \left\{J \geq 0: P_{\infty}(\beta, J, h)>0\right\} .
$$

It turns out that if $P_{\infty}(\beta, J, h)$ is replaced by $\widetilde{P}_{\infty}(\beta, J, h)$ in the definition above, the value of $J_{c}(\beta, h)$ is unchanged, again by the corollary to Theorem 3.1.

Remark. For $d \geq 2$ and $q$ sufficiently large, $P_{\infty}(\beta, J, 0)$ jumps from zero below $J_{c}$ to a strictly positive number at $J_{c}$. This corresponds to the so-called temperature driven first order phase transition in the Potts model, whose existence was first proved in [19].

Theorem 2.5 Let $\beta \geq 0$ and $h_{m} \in \mathbb{R}, m=1, \ldots, q$.

(i) For all $J \geq 0$, there is at most one ES Gibbs measure with no infinite cluster.

(ii) If $P_{\infty}(\beta, J, h)=0$, then $\left|\mathcal{G}^{\mathrm{ES}}\right|=\left|\mathcal{G}^{\mathrm{RC}}\right|=1$. In particular, $\left|\mathcal{G}^{\mathrm{ES}}\right|=\left|\mathcal{G}^{\mathrm{RC}}\right|=1$ if $J<J_{c}$.

(iii) If $P_{\infty}(\beta, J, h)>0$, then the states $\mu_{m}^{\mathrm{ES}}, m \in Q_{\max }(h)$, are extremal translation invariant ES Gibbs states with $\mu_{m}^{\mathrm{ES}}\left(\mathcal{A}_{1, m}^{\infty}\right)=1$. In particular, there are at least $q_{0}=\left|Q_{\max }(h)\right|$ different extremal translation invariant ES Gibbs states.

As mentioned above, the percolation probability $P_{\infty}(\beta, J, h)$ is nondecreasing in $J$. The last statement of the theorem therefore implies that there are at least $q_{0}$ extremal translation invariant ES Gibbs states when $J>J_{c}$. This raises the question of whether for $\left|Q_{\max }(h)\right|=1$ the ES Gibbs state is unique above $J_{c}$. As the next theorem shows, this is indeed the case, at least if $d=2$.

Theorem 2.6 Let $\beta \geq 0$, and $h_{m} \in \mathbb{R}, m=1, \ldots, q$, and $d=2$.

(i) If $J \neq J_{c}$, then $\left|\mathcal{G}^{\mathrm{RC}}\right|=1$ and $P_{\infty}(\beta, J, h)=\widetilde{P}_{\infty}(\beta, J, h)$.

(ii) If $J \neq J_{c}$ and, in addition, $\left|Q_{\max }(h)\right|=1$, then $\left|\mathcal{G}^{\mathrm{ES}}\right|=1$.

Remarks. (i) For the Ising model, the condition $\left|Q_{\max }(h)\right|=1$ means that $h \neq 0$. Together with FKG, the Lee-Yang theorem then implies that the claim (ii) is valid for $d \geq 2$ and all $J \geq 0$, including $J=J_{c}$. Even though one might conjecture that this statement holds for arbitrary $q$, since only one spin direction is preferred if $\left|Q_{\max }(h)\right|=1$, this is in fact not true. Indeed, we show in [3] that the $q$-state Potts model has two coexisting phases at $J_{c}$ for sufficiently small fields preferring one of the $q$ values $m \in\{1, \ldots, q\}$ over all others, provided $q$ is sufficiently large. However, we believe that for $J \neq J_{c},\left|Q_{\max }(h)\right|=1$ does imply uniqueness for all $q$, even when $d>2$. 
(ii) Theorem 2.6(i) and part of the statement in Theorems 2.5(ii) refer to the RC model itself, and not the relationship between the ES and the RC model. As we will see in the proofs of Theorems 2.5 and 2.6 in Sections 8 and 9, these statements remain true in the more general context of the GRC model introduced in Section 1.

\section{Monotonicity and Uniqueness of the Infinite Cluster}

In this section, we define the generalized random cluster (GRC) model, and formulate several results concerning the FKG properties and uniqueness of the infinite cluster in this model. The GRC measure $\mu_{\Lambda \text {,free }}^{\mathrm{GRC}}$ is obtained by normalizing the weights

$$
W_{\Lambda, \text { free }}^{\mathrm{GRC}}(\boldsymbol{\eta})=\left(e^{\beta J}-1\right)^{|\boldsymbol{\eta}|} \prod_{C(\boldsymbol{\eta})} \Theta_{\text {free }}(C(\boldsymbol{\eta})),
$$

for any $\boldsymbol{\eta} \in\{0,1\}^{\mathbb{B}_{0}(\Lambda)}$. Here $|\boldsymbol{\eta}|$ is the number of bonds in the set $\left\{b \in \mathbb{B}_{0}(\Lambda): \eta_{b}=1\right\}$, the product runs over all connected components $C(\boldsymbol{\eta})$ of the $\operatorname{graph}^{5}\left(\Lambda, \mathbb{B}_{\mathrm{occ}}(\boldsymbol{\eta}) \cap \mathbb{B}_{0}(\Lambda)\right)$, and

$$
\Theta_{\text {free }}(C)=\sum_{m=1}^{q} q_{m} e^{\beta h_{m}|\mathbb{V}(C)|},
$$

for any connected component $C$. The factors $q_{m}, m=1, \ldots, q$, are assumed to be positive real numbers such that

$$
\sum_{m \in Q_{\max }} q_{m} \geq 1
$$

Similarly, the measure $\mu_{\Lambda, m}^{\mathrm{GRC}}$ is obtained by normalizing the weights $W_{\Lambda, m}^{\mathrm{GRC}}$ defined for any $\boldsymbol{\eta} \in\{0,1\}^{\mathbb{B}(\Lambda)}$ by the formula

$$
W_{\Lambda, m}^{\mathrm{GRC}}(\boldsymbol{\eta})=\left(e^{\beta J}-1\right)^{|\boldsymbol{\eta}|} \prod_{C(\boldsymbol{\eta})} \Theta_{\Lambda, m}(C(\boldsymbol{\eta})),
$$

where $|\boldsymbol{\eta}|$ now stands for the number of bonds in the set $\left\{b \in \mathbb{B}(\Lambda): \eta_{b}=1\right\}$, the product runs over all connected components $C(\boldsymbol{\eta})$ of the graph $\left(\bar{\Lambda}, \mathbb{B}_{\text {occ }}(\boldsymbol{\eta}) \cap \mathbb{B}(\Lambda)\right), \bar{\Lambda}=\Lambda \cup \partial \Lambda$, and $\Theta_{\Lambda, m}(C)$ is defined as

$$
\Theta_{\Lambda, m}(C)= \begin{cases}\Theta_{\text {free }}(C) & \mathbb{V}(C) \cap \Lambda^{\mathrm{c}}=\emptyset \\ e^{\beta h_{m}|\mathbb{V}(C)|} & \text { otherwise. }\end{cases}
$$

As already pointed out for RC measures, the measures $\mu_{\Lambda, m}^{\mathrm{GRC}}$ are identical for all values $m \in$ $Q_{\max }(h)$; we will use $\mu_{\Lambda, \text { maxwir }}^{\mathrm{GRC}}$ to denote any of them. Note also that the definitions (3.4) and (3.5) reduce to the standard definition of wired measures for non-integer $q$ when $h_{m} \equiv 0$.

Finally, one can directly extend the definition (2.9) to get the weights $W_{\mathbb{B}}^{\mathrm{GRC}}\left(\boldsymbol{\eta}_{\mathbb{B}} \mid \boldsymbol{\eta}_{\mathbb{B}^{\mathrm{c}}}\right)$,

$$
W_{\mathbb{B}}^{\mathrm{GRC}}\left(\boldsymbol{\eta}_{\mathbb{B}} \mid \boldsymbol{\eta}_{\mathbb{B c}}\right)=\left(e^{\beta J}-1\right)^{\left|\mathbb{B}_{\mathrm{occ}}\left(\boldsymbol{\eta}_{\mathbb{B}}\right) \cap \mathbb{B}\right|} \prod_{C(\boldsymbol{\eta}): \mathbb{V}(C(\boldsymbol{\eta})) \cap \mathbb{V}(\mathbb{B}) \neq \emptyset} \sum_{m=1}^{q} q_{m} e^{-\beta\left(h_{\max }-h_{m}\right)|\mathbb{V}(C(\boldsymbol{\eta}))|},
$$

yielding the measures $\mu_{\mathbb{B}}^{\mathrm{GRC}}\left(\boldsymbol{\eta}_{\mathbb{B}} \mid \boldsymbol{\eta}_{\mathbb{B}^{c}}\right)$ that define GRC Gibbs states with the help of DLR equations of the type (2.12). GRC limit states are defined analogously to RC limit states, see Remark (i) following Theorem 2.3.

\footnotetext{
${ }^{5}$ We recall that $\mathbb{B}_{\text {occ }}(\boldsymbol{\eta})$ denotes the set of bonds $b$ with $\eta_{b}=1$.
} 
Remarks. (i) It is easy to see that if we take $q_{m}=1, m=1, \ldots, q$, then the measures $\mu_{\Lambda \text {,free }}^{\mathrm{GRC}}$ and $\mu_{\Lambda, m}^{\mathrm{GRC}}$ are just the $\mathrm{RC}$ marginals $\mu_{\Lambda, \text { free }}^{\mathrm{RC}}$ and $\mu_{\Lambda, m}^{\mathrm{RC}}$, respectively.

(ii) It is instructive to consider the effects of particular boundary conditions on the measure with weights (3.6). If we take $\mathbb{B}=\mathbb{B}_{0}(\Lambda)$ and $\boldsymbol{\eta}_{\mathbb{B}^{c}} \equiv 0$, then we get the free measure $\mu_{\Lambda, \text { free. If, }}^{\mathrm{GRC}}$, on the other hand, we take $\mathbb{B}=\mathbb{B}(\Lambda)$ and $\boldsymbol{\eta}_{\mathbb{B}^{c}} \equiv 1$, then we get the wired measure $\mu_{\Lambda \text {,maxwir }}^{\text {GRC }}$, provided $\Lambda^{\mathrm{c}}$ is connected. If $\Lambda^{\mathrm{c}}$ is not connected, i.e., if $\Lambda$ contains "holes," then the boundaries of these holes will be not be wired to each other. In this case, it will often be convenient to introduce additional "ghost" bonds linking all of the components of the boundary. If, in addition to the bonds in $\mathbb{B}(\Lambda)^{\mathrm{c}}$, the ghost bonds are occupied, we get the maxwired state also in this case.

(iii) Recall that in the standard RC model without magnetic fields it is possible to view the wired state as a free state on a modified graph in which all of the boundary sites in $\partial \Lambda$ have been identified. However, in the case of general external fields, the two prescriptions produce different states, i.e., setting all the sites at the boundary to a particular value produces a different state from the free state on a graph in which all boundary sites have been identified. In the former case, the collection $\left\{C_{i}\right\}$ of all components of $\left(\Lambda \cup \partial \Lambda, \mathbb{B}_{\text {occ }}(\boldsymbol{\eta}) \cap \mathbb{B}(\Lambda)\right)$ that touch the boundary acquires the weight $e^{\beta h_{\max } \sum_{i}\left|\mathbb{V}\left(C_{i}\right)\right|}$, while in the latter case, it acquires the weight $\sum_{m} e^{\beta h_{m}\left(1+\sum_{i}\left|\mathbb{V}\left(C_{i}\right) \backslash \Lambda^{\mathrm{c}}\right|\right)}$.

Note that it is the former prescription that we use to define the $m$-wired GRC measure. This measure is natural for two reasons: it is the marginal of the corresponding ES measure if all $q_{m}$ 's are one, and, for $m \in Q_{\max }(h)$, this measure is maximal in the FKG order, whereas the alternative one is not, at least in a finite volume.

To state our results on FKG properties, we introduce the standard partial order $\prec$ on $\{0,1\}^{\mathbb{B}\left(\mathbb{Z}^{d}\right)}$ by setting $\boldsymbol{\eta} \prec \boldsymbol{\eta}^{\prime}$ whenever $\eta_{b} \leq \eta_{b}^{\prime}$ for every $b \in \mathbb{B}\left(\mathbb{Z}^{d}\right)$. Since we shall also study monotonicity properties in dependence on $\left(h_{m}\right)$ we need to introduce a partial order on the external fields. Given two sets of fields $\left(h_{m}\right)$ and $\left(h_{m}^{\prime}\right)$, we define

$$
\left(h_{m}\right) \preccurlyeq\left(h_{m}^{\prime}\right) \text { iff } h_{k}-h_{l} \leq h_{k}^{\prime}-h_{l}^{\prime} \text { for all } k, l=1, \ldots, q \text { with } h_{k}-h_{l}>0 \text {. }
$$

Note that $\preccurlyeq$ is indeed a partial order on $q$-tuples of real numbers, in particular, $\left(h_{m}\right) \preccurlyeq\left(h_{m}^{\prime}\right)$ and $\left(h_{m}^{\prime}\right) \preccurlyeq\left(h_{m}^{\prime \prime}\right)$ imply $\left(h_{m}\right) \preccurlyeq\left(h_{m}^{\prime \prime}\right)$.

Recall the following definition:

Definition. Let $\Omega$ be a measurable space endowed with the partial order $\prec$. Then a measure $\mu$ on $\Omega$ is said to be $F K G$ if $\mu(F G) \geq \mu(F) \mu(G)$ for all measurable functions $F, G: \Omega \rightarrow \mathbb{R}$ that are increasing with respect to $\prec$. Moreover, if $\Omega$ is of the form $\Omega=\times_{b \in \mathbb{B}} \Omega_{b}$, then $\mu$ is said to be strong $F K G$ if $\mu(\cdot \mid A)$ is $F K G$ for all cylinder events of the form $A=\left\{\boldsymbol{\eta}: \eta_{b}=\alpha_{b} \forall b \in \widetilde{\mathbb{B}}\right\}$, where $\widetilde{\mathbb{B}} \subset \mathbb{B}$ is finite and $\alpha_{b} \in \Omega_{b}$ for all $b \in \widetilde{\mathbb{B}}$.

Theorem 3.1 Let $\beta \geq 0, J \geq 0, h_{m} \in \mathbb{R}$ and $q_{m}>0, m=1, \ldots, q$, and suppose that the parameters $q_{m}$ obey the condition (3.3). Then:

(i) For each finite $\Lambda \subset \mathbb{Z}^{d}$, the measures $\mu_{\Lambda \text {,free }}^{\mathrm{GRC}}$ and $\mu_{\Lambda, \text { maxwir }}^{\mathrm{GRC}}$ are strong $F K G$.

(ii) For each quasilocal function $f$, the limits

$$
\mu_{\operatorname{maxwir}}^{\mathrm{GRC}}(f)=\lim _{\Lambda \nearrow \mathbb{Z}^{d}} \mu_{\Lambda, \text { maxwir }}^{\mathrm{GRC}}(f)
$$


and

$$
\mu_{\text {free }}^{\mathrm{GRC}}(f)=\lim _{\Lambda \nearrow \mathbb{Z}^{d}} \mu_{\Lambda, \text { free }}^{\mathrm{GRC}}(f)
$$

exist and are translation invariant.

(iii) Let $\mu$ be a GRC limit state or a GRC Gibbs state. Then

$$
\mu_{\text {free }}^{\mathrm{GRC}} \underset{\mathrm{FKG}}{\leq} \mu \underset{\mathrm{FKG}}{\leq} \mu_{\text {maxwir }}^{\mathrm{GRC}}
$$

(iv) Suppose $J_{1}<J_{2}$. Let let $\mu_{\mathrm{maxwir}}^{\mathrm{GRC}, J_{1}}$ denote the wired state at $J=J_{1}$ and let $\mu_{\mathrm{free}}^{\mathrm{GRC}, J_{2}}$ denote the free state at $J=J_{2}$. Then

$$
\mu_{\text {maxwir }}^{\mathrm{GRC}, J_{1}}(\cdot) \underset{\mathrm{FKG}}{\leq} \mu_{\text {free }}^{\mathrm{GRC}, J_{2}}(\cdot) .
$$

(v) Let $\left(h_{m}\right) \preccurlyeq\left(h_{m}^{\prime}\right)$ be two sets of external fields. Then

$$
\begin{aligned}
& \mu_{\text {free }}^{\mathrm{GRC},\left(h_{m}\right)}(\cdot) \underset{\mathrm{FKG}}{\leq} \mu_{\text {free }}^{\mathrm{GRC},\left(h_{m}^{\prime}\right)}(\cdot) \\
& \mu_{\text {maxwir }}^{\mathrm{GRC},\left(h_{m}\right)}(\cdot) \underset{\mathrm{FKG}}{\leq} \mu_{\text {maxwir }}^{\mathrm{GRC},\left(h_{m}^{\prime}\right)}(\cdot) .
\end{aligned}
$$

Remark. Note that (3.11) can be extended via (3.10) to any pair of GRC Gibbs measures at $J=J_{1}$, resp. $J=J_{2}$.

The following corollary is an immediate consequence of the above theorem. Before stating it, we recall the definitions $(2.21),(2.23)$ and $(2.22)$ of $P_{\infty}(\beta, J, h), J_{c}$, and $\widetilde{P}_{\infty}(\beta, J, h)$, respectively. For the GRC measures considered here, the definitions (2.21) and (2.22) are obviously modified by replacing the space $\widetilde{\mathcal{G}}^{\mathrm{RC}}$ of translation invariant $\mathrm{RC}$ Gibbs states by the space $\widetilde{\mathcal{G}}^{\mathrm{GRC}}$ of translation invariant GRC Gibbs states.

Corollary. Let $\beta \geq 0, J \geq 0, h_{m} \in \mathbb{R}$ and $q_{m}>0, m=1, \ldots, q$, and suppose that the parameters $q_{m}$ obey the condition (3.3). Then:

(i) $P_{\infty}(\beta, J, h)=\mu_{\operatorname{maxwir}}^{\mathrm{GRC}}\left(\left|C_{0}\right|=\infty\right)$.

(ii) $\widetilde{P}_{\infty}(\beta, J, h)=\mu_{\text {free }}^{\mathrm{GRC}}\left(\left|C_{0}\right|=\infty\right)$.

(iii) $J \mapsto P_{\infty}(\beta, J, h)$ is a nondecreasing, right continuous function.

(iv) $J \mapsto \widetilde{P}_{\infty}(\beta, J, h)$ is a nondecreasing function, which is continuous and equal to $P_{\infty}(\beta, J, h)$ whenever $J \mapsto P_{\infty}(\beta, J, h)$ is continuous.

(v) $P_{\infty}(\beta, J, h)=\widetilde{P}_{\infty}(\beta, J, h)=0$ if $J<J_{c}$, while both $P_{\infty}(\beta, J, h)>0$ and $\widetilde{P}_{\infty}(\beta, J, h)>0$ if $J>J_{c}$.

The next theorem is the only statement in this section that cannot be generalized to the GRC models.

Theorem 3.2 Let $\beta \geq 0, J \geq 0$, and $h_{m} \in \mathbb{R}, m=1, \ldots, q$. Let $\nu \in \mathcal{G}^{\mathrm{ES}}$ be arbitrary and let $\mu$ denote its $\boldsymbol{\eta}$-marginal. Then

$$
\mu(\cdot) \underset{\mathrm{FKG}}{\leq} \mu_{\operatorname{maxwir}}^{\mathrm{RC}}(\cdot) .
$$

If, in addition, either $\left|Q_{\max }(h)\right|=1$ or $\mu\left(N_{\infty} \leq 1\right)=1$, then

$$
\mu(\cdot) \underset{\mathrm{FKG}}{\geq} \mu_{\mathrm{free}}^{\mathrm{RC}}(\cdot) \text {. }
$$


The following theorem states our results on the uniqueness of the infinite cluster.

Theorem 3.3 Let $\beta \geq 0, J \geq 0, h_{m} \in \mathbb{R}$ and $q_{m}>0, m=1, \ldots, q$, and suppose that the parameters $q_{m}$ obey the condition (3.3). Then all translation invariant GRC Gibbs states and all translation invariant GRC limit states have at most one infinite cluster with probability one.

Remark. We will prove the above theorem by first establishing the so-called finite energy condition for $\mu$, and then using the results of [6]. Unfortunately, we were unable to use this strategy to prove uniqueness of the infinite cluster for random cluster marginals of translation invariant ES Gibbs measures. In fact, it is not hard to see that there are ES Gibbs states whose random cluster marginals do not satisfy the finite energy condition. While these counterexamples stem from non-translation invariant ES Gibbs states obtained by so-called Dobrushin boundary conditions, we do not see how to use the additional assumption of translation invariance to get a proof of the finite energy condition.

In Section 7 we will use the uniqueness of the infinite cluster to prove that the finite volume specifications of $\mu_{\text {free }}^{\mathrm{GRC}}$ and $\mu_{\mathrm{maxwir}}^{\mathrm{GRC}}$, and more generally of any translation invariant GRC limit state, are "almost surely quasilocal" in the terminology of [21] and [16]. As a corollary of this statement, we will prove the following result.

Corollary. Let $\beta \geq 0, J \geq 0, h_{m} \in \mathbb{R}$ and $q_{m}>0, m=1, \ldots, q$, and suppose that the parameters $q_{m}$ obey the condition (3.3). Then all translation invariant GRC limit states are GRC Gibbs states.

The last theorem in this section addresses the question under which conditions the conditional expectations of a GRC Gibbs state $\mu$ are given by the measures $\mu_{\mathbb{B}}^{\mathrm{GRC}}\left(\cdot \mid \boldsymbol{\eta}_{\mathbb{B}^{c}}\right)$.

Theorem 3.4 Let $\beta \geq 0, J \geq 0, h_{m} \in \mathbb{R}$ and $q_{m}>0, m=1, \ldots, q$, and suppose that the parameters $q_{m}$ obey the condition (3.3). Let $\mu$ be a GRC Gibbs state with $\mu\left(N_{\infty} \leq 1\right)=1$, let $\mathbb{B}$ be a finite subset of $\mathbb{B}_{0}\left(\mathbb{Z}^{d}\right)$, and let $f$ be a cylinder functions depending only on the configuration $\boldsymbol{\eta}_{\mathbb{B}}$. Then

$$
\mu\left(f \mid \boldsymbol{\eta}_{\mathbb{B}^{c}}\right)=\mu_{\mathbb{B}}^{\mathrm{GRC}}\left(f \mid \boldsymbol{\eta}_{\mathbb{B}^{c}}\right) \quad \mu \text {-a.s. }
$$

\section{FKG Properties of Generalized Random Cluster Measures}

In this section we prove Theorem 3.1. In the process we formulate and prove a lemma concerning monotonicity of GRC states in the volume (Lemma 4.1). We will also formulate and prove a second result (Lemma 4.2) concerning domination of states with general boundary conditions, which will be used in the proof of Theorem 3.2 in the next section.

Proof of Theorem 3.1(i). We consider $\Lambda$ to be fixed and omit it temporarily from the notation. In order to prove the strong FKG property of $\mu_{\Lambda, \text { free }}^{\mathrm{GRC}}$ and $\mu_{\Lambda, m}^{\mathrm{GRC}}$, let us recall a necessary and sufficient condition [10], the so-called lattice condition

$$
W_{\text {free }}^{\mathrm{GRC}}\left(\boldsymbol{\eta}^{(1)} \vee \boldsymbol{\eta}^{(2)}\right) W_{\text {free }}^{\mathrm{GRC}}\left(\boldsymbol{\eta}^{(1)} \wedge \boldsymbol{\eta}^{(2)}\right) \geq W_{\text {free }}^{\mathrm{GRC}}\left(\boldsymbol{\eta}^{(1)}\right) W_{\text {free }}^{\mathrm{GRC}}\left(\boldsymbol{\eta}^{(2)}\right)
$$

for any pair of configurations $\boldsymbol{\eta}^{(1)}$ and $\boldsymbol{\eta}^{(2)}$, and similarly for $W_{m}^{\mathrm{GRC}}$. Here $\boldsymbol{\eta}^{(1)} \vee \boldsymbol{\eta}^{(2)}$ denotes the maximum and $\boldsymbol{\eta}^{(1)} \wedge \boldsymbol{\eta}^{(2)}$ the minimum of $\boldsymbol{\eta}^{(1)}$ and $\boldsymbol{\eta}^{(2)}$. 
It turns out that to verify (4.1), it suffices to consider $\boldsymbol{\eta}^{(1)}$ and $\boldsymbol{\eta}^{(2)}$ that differ just at two bonds. Indeed (see e.g., [7]), let

$$
\mathcal{R}(\boldsymbol{\zeta}, \boldsymbol{\eta})=\frac{W_{\text {free }}^{\mathrm{GRC}}(\boldsymbol{\zeta} \vee \boldsymbol{\eta})}{W_{\text {free }}^{\mathrm{GRC}}(\boldsymbol{\zeta})}
$$

and note that (4.1) can be rewritten as $\mathcal{R}\left(\boldsymbol{\eta}^{(1)}, \boldsymbol{\eta}^{(2)}\right) \geq \mathcal{R}\left(\boldsymbol{\eta}^{(1)} \wedge \boldsymbol{\eta}^{(2)}, \boldsymbol{\eta}^{(2)}\right)$. Hence, the lattice condition (4.1) is true once we verify that $\mathcal{R}(\boldsymbol{\zeta}, \boldsymbol{\eta})$ is increasing in $\boldsymbol{\zeta}$, for any fixed $\boldsymbol{\eta}$. Let us introduce, for any bond $b$, the configuration $\boldsymbol{\eta}^{(b)}$ by setting $\eta_{b}^{(b)}=1$ and $\eta_{b^{\prime}}^{(b)}=0$ for any $b^{\prime} \neq b$. Ordering the set $\mathbb{B}_{\text {occ }}(\boldsymbol{\eta})$ into a sequence $\left(b_{1}, \ldots, b_{\left|\mathbb{B}_{\text {occ }}(\boldsymbol{\eta})\right|}\right)$, we have

$$
\mathcal{R}(\boldsymbol{\zeta}, \boldsymbol{\eta})=\prod_{k=1}^{\left|\mathbb{B}_{\text {occ }}(\boldsymbol{\eta})\right|} \mathcal{R}\left(\boldsymbol{\zeta} \vee \boldsymbol{\eta}^{\left(b_{1}\right)} \vee \cdots \vee \boldsymbol{\eta}^{\left(b_{k-1}\right)}, \boldsymbol{\eta}^{\left(b_{k}\right)}\right)
$$

Hence, it suffices to prove monotonicity of $\mathcal{R}(\boldsymbol{\zeta}, \boldsymbol{\eta})$ for any $\boldsymbol{\eta}$ that is zero except possibly at one bond. Moreover, it suffices to prove the growth when flipping $\zeta$ at a single bond from 0 to 1, i.e., $\boldsymbol{\zeta}$ with $\zeta_{b}=0$ to $\boldsymbol{\zeta}^{b}=\boldsymbol{\zeta} \vee \boldsymbol{\eta}^{(b)}$. The verification of the needed bound, $\mathcal{R}\left(\boldsymbol{\zeta}^{b}, \boldsymbol{\eta}^{\left(b^{\prime}\right)}\right) \geq \mathcal{R}\left(\boldsymbol{\zeta}, \boldsymbol{\eta}^{\left(b^{\prime}\right)}\right)$, for any pair of bonds $b$ and $b^{\prime}$, now boils down to the special case of (4.1) with $\boldsymbol{\eta}^{(1)}=\boldsymbol{\zeta}^{b}$ and $\boldsymbol{\eta}^{(2)}=\boldsymbol{\zeta} \vee \boldsymbol{\eta}^{\left(b^{\prime}\right)}$ that differ only at bonds $b$ and $b^{\prime}$. Since $\boldsymbol{\eta}^{(1)}=\boldsymbol{\eta}^{(2)}$ if $b=b^{\prime}$, we may further assume without loss of generality that $b \neq b^{\prime}$.

Let thus $\boldsymbol{\eta}^{(1)}$ and $\boldsymbol{\eta}^{(2)}$ be such that

$$
\begin{array}{ll}
\eta_{b}^{(1)}=\eta_{b}^{(2)} & b \neq b_{1}, b_{2} \\
\eta_{b_{1}}^{(1)}=\eta_{b_{2}}^{(2)}=0 & \eta_{b_{2}}^{(1)}=\eta_{b_{1}}^{(2)}=1 .
\end{array}
$$

Since the number of 1-bonds is equal on both sides of (4.1), the nontrivial issue is therefore to check (4.1) for the product over the connected components. Let us suppose, without loss of generality, that there exist disjoint connected components $A_{1}$ and $A_{2}$ of $\boldsymbol{\eta}^{(1)} \wedge \boldsymbol{\eta}^{(2)}$ (possibly isolated sites) that become connected when $b_{1}$ is flipped from 0 to 1 , and, similarly, $B_{1}$, $B_{2}$ for the components connected by flipping $b_{2}$. (The only other possibility is that both endpoints of $b_{1}$, or alternatively $b_{2}$, lie in a single component of $\boldsymbol{\eta}^{(1)} \wedge \boldsymbol{\eta}^{(2)}$, in which case the two sides of (4.1) are equal.) With this proviso, there are only three generic situations:

(a) $\mathbb{V}\left(A_{1}\right) \cup \mathbb{V}\left(A_{2}\right)$ is disjoint from $\mathbb{V}\left(B_{1}\right) \cup \mathbb{V}\left(B_{2}\right)$,

(b) $\mathbb{V}\left(A_{1}\right)=\mathbb{V}\left(B_{1}\right)$ but $\mathbb{V}\left(A_{2}\right) \cap \mathbb{V}\left(B_{2}\right)=\emptyset$,

(c) $\mathbb{V}\left(A_{1}\right)=\mathbb{V}\left(B_{1}\right)$ and $\mathbb{V}\left(A_{2}\right)=\mathbb{V}\left(B_{2}\right)$.

We will prove (4.1) separately for (a), (b), and (c). For notational brevity, we use $\Theta(C)$ for both $\Theta_{\text {free }}(C)$ and $\Theta_{m}(C)$.

In the case (a) both sides of (4.1) reduce to the same term

$$
\Theta\left(A_{1} \cup A_{2}\right) \Theta\left(B_{1} \cup B_{2}\right) \Theta\left(A_{1}\right) \Theta\left(A_{2}\right) \Theta\left(B_{1}\right) \Theta\left(B_{2}\right) .
$$

Hence, (4.1) is fulfilled with the equality sign.

Next, consider (b). We denote by $C$ the common component (i.e., $C=A_{1}=B_{1}$ ) and use $A$ and $B$ to denote the other components. Then (4.1) boils down to the inequality

$$
\Theta(C) \Theta(C \cup A \cup B) \geq \Theta(C \cup A) \Theta(C \cup B) .
$$


GIBBS STATES OF GRAPHICAL REPRESENTATIONS IN THE POTTS MODEL

Let us first consider the free boundary conditions. Using, for any $m \in\{1, \ldots, q\}$, the notation

$$
\begin{aligned}
& a_{m}=e^{\beta h_{m}|\mathbb{V}(A)|}, \\
& b_{m}=e^{\beta h_{m}|\mathbb{V}(B)|}, \\
& c_{m}=e^{\beta h_{m}|\mathbb{V}(C)|},
\end{aligned}
$$

the condition (4.6) is equivalent to

$$
\left(\sum_{m=1}^{q} q_{m} c_{m}\right)\left(\sum_{m^{\prime}=1}^{q} q_{m^{\prime}} a_{m^{\prime}} b_{m^{\prime}} c_{m^{\prime}}\right) \geq\left(\sum_{m=1}^{q} q_{m} a_{m} c_{m}\right)\left(\sum_{m^{\prime}=1}^{q} q_{m^{\prime}} b_{m^{\prime}} c_{m^{\prime}}\right) .
$$

Let us assume that the fields are ordered in an increasing order, $h_{1} \leq h_{2} \leq \cdots \leq h_{q}$. As a consequence, $a_{1} \leq a_{2} \leq \cdots \leq a_{q}$ and $b_{1} \leq b_{2} \leq \cdots \leq b_{q}$. By writing the expression (4.8) as an inequality for a bilinear form in $q_{m} c_{m} q_{m^{\prime}} c_{m^{\prime}}$, the sufficient requirement that all the independent coefficients of this form be non-negative reduces to

$$
\left(a_{m}-a_{m^{\prime}}\right)\left(b_{m}-b_{m^{\prime}}\right) \geq 0 \quad \forall m, m^{\prime}
$$

which is immediate by our preceding assumptions.

Turning to $\bar{m}$-wired boundary conditions, $\bar{m} \in Q_{\max }(h)$, we will distinguish several cases. If $\mathbb{V}(A) \cap \Lambda^{\mathrm{c}}=\emptyset, \mathbb{V}(B) \cap \Lambda^{\mathrm{c}}=\emptyset$, as well as $\mathbb{V}(C) \cap \Lambda^{\mathrm{c}}=\emptyset$, we have exactly the same situation as for free boundary conditions. If $\mathbb{V}(C) \cap \Lambda^{\mathrm{c}} \neq \emptyset$, both sides of (4.6) are equal to $c_{\bar{m}} a_{\bar{m}} b_{\bar{m}} c_{\bar{m}}$. If $\mathbb{V}(A) \cap \Lambda^{\mathrm{c}}=\emptyset, \mathbb{V}(C) \cap \Lambda^{\mathrm{c}}=\emptyset$, and $\mathbb{V}(B) \cap \Lambda^{\mathrm{c}} \neq \emptyset$, we need to show that

$$
\left(\sum_{m=1}^{q} q_{m} c_{m}\right) a_{\bar{m}} b_{\bar{m}} c_{\bar{m}} \geq\left(\sum_{m=1}^{q} q_{m} a_{m} c_{m}\right) b_{\bar{m}} c_{\bar{m}}
$$

This follows once we realize that $h_{\bar{m}}=h_{\max }$ implies $a_{m} \leq a_{\bar{m}}$ for any $m$. Similarly with the role of $A$ and $B$ interchanged. Finally, if $\mathbb{V}(C) \cap \Lambda^{\mathrm{c}}=\emptyset$, but $\mathbb{V}(A) \cap \Lambda^{\mathrm{c}} \neq \emptyset$ and $\mathbb{V}(B) \cap \Lambda^{\mathrm{c}} \neq \emptyset$, we have to verify that

$$
\left(\sum_{m=1}^{q} q_{m} c_{m}\right) a_{\bar{m}} b_{\bar{m}} c_{\bar{m}} \geq a_{\bar{m}} c_{\bar{m}} b_{\bar{m}} c_{\bar{m}}
$$

This is clearly true if we use the assumption that $\sum_{m \in Q_{\max }(h)} q_{m} \geq 1$ and the fact that $c_{m}=c_{\bar{m}}$ whenever $m \in Q_{\max }(h)$.

It remains to establish (4.1) under (c). In this case, there are only two components in the game: $A$ and $B$. Inequality (4.1) is then implied by $\Theta(A \cup B) \leq \Theta(A) \Theta(B)$. Let us use the definitions (4.7) of $a_{m}$ and $b_{m}$. We consider three cases. First, in the case of either free boundary conditions, or wired boundary conditions with the additional conditions $\mathbb{V}(A) \cap \Lambda^{\mathrm{c}}=$ $\emptyset$ and $\mathbb{V}(B) \cap \Lambda^{\mathrm{c}}=\emptyset$, the relation we want boils down to the inequality

$$
\sum_{m=1}^{q} q_{m} a_{m} b_{m} \leq\left(\sum_{m=1}^{q} q_{m} a_{m}\right)\left(\sum_{m^{\prime}=1}^{q} q_{m^{\prime}} b_{m^{\prime}}\right)
$$

which is obviously satisfied since $b_{m} \leq \sum_{m^{\prime} \in Q \max (h)} q_{m^{\prime}} b_{\bar{m}}$. Second, for wired boundary conditions under the additional conditions $\mathbb{V}(A) \cap \Lambda^{\mathrm{c}}=\emptyset$ and $\mathbb{V}(B) \cap \Lambda^{\mathrm{c}} \neq \emptyset$, we get the manifestly correct inequality

$$
a_{\bar{m}} b_{\bar{m}} \leq\left(\sum_{m=1}^{q} q_{m} a_{m}\right) b_{\bar{m}}
$$


Finally, for wired boundary conditions with the additional conditions $\mathbb{V}(A) \cap \Lambda^{\mathrm{c}} \neq \emptyset$ and $\mathbb{V}(B) \cap \Lambda^{\mathrm{c}} \neq \emptyset$, we get the identity $a_{\bar{m}} b_{\bar{m}}=a_{\bar{m}} b_{\bar{m}}$.

Remark. The necessity of $h_{m}=h_{\max }$, for the strong FKG property of $\mu_{\Lambda, m}^{\mathrm{GRC}}$ to hold, arises from (4.6). Namely, suppose that $B$ connects to the boundary (i.e., $\mathbb{V}(B) \cap \Lambda^{\mathrm{c}} \neq \emptyset$ ), whereas $A$ and $C$ do not. Then (4.6) reduces to (4.10). It is not difficult to convince oneself that choosing $C$ sufficiently large one can make (4.10) be satisfied for all $A$ only when $a_{\bar{m}}=\max _{m} a_{m}$. Consequently, $h_{\bar{m}}$ must be equal to $h_{\max }$ for the lattice condition (4.1) or, equivalently, the strong FKG condition to hold.

Lemma 4.1 Let $\beta \geq 0, J \geq 0, h_{m} \in \mathbb{R}$ and $q_{m}>0, m=1, \ldots, q$, and suppose that the parameters $q_{m}$ obey the condition (3.3). Further, let $\Lambda \subset \Delta \subset \mathbb{Z}^{d}$ be two finite sets. Then

$$
\mu_{\Lambda, \text { free }}^{\mathrm{GRC}}(\cdot) \underset{\mathrm{FKG}}{\leq} \mu_{\Delta, \text { free }}^{\mathrm{GRC}}(\cdot)
$$

and

$$
\mu_{\Lambda, \text { maxwir }}^{\mathrm{GRC}}(\cdot) \underset{\mathrm{FKG}}{\geq} \mu_{\Delta, \text { maxwir }}^{\mathrm{GRC}}(\cdot) .
$$

Proof. Using Theorem 3.1(i), the inequality (4.14) follows immediately from the fact that

$$
\mu_{\Lambda, \text { free }}^{\mathrm{GRC}}(\cdot)=\mu_{\Delta, \text { free }}^{\mathrm{GRC}}\left(\cdot \mid \mathcal{D}_{\Lambda}\right),
$$

where $\mathcal{D}_{\Lambda}$ is the FKG decreasing event

$$
\mathcal{D}_{\Lambda}=\left\{\boldsymbol{\eta}: \eta_{b}=0 \forall b \in \mathbb{B}_{0}(\Lambda)^{\mathrm{c}}\right\} .
$$

For maxwired boundary conditions, the proof is more complicated, since conditioning on the FKG increasing event

$$
\mathcal{O}_{\Lambda}=\left\{\boldsymbol{\eta}: \eta_{b}=1 \forall b \in \mathbb{B}(\Lambda)^{\mathrm{c}}\right\}
$$

leads to the state $\mu_{\Lambda \text {,maxwir }}^{\mathrm{GRC}}$ only if $\Lambda$ is a volume without "holes", i.e. if $\Lambda^{\mathrm{c}}$ has only one (infinite) component. If $\Lambda^{\mathrm{c}}$ has finite components $H_{1}, \ldots, H_{k}$, we use the following trick: for each "hole" $H_{i}$, we introduce an additional bond $b_{i}$ with one endpoint in $H_{i}$ and the other in $\Delta^{\mathrm{c}}$. Setting

$$
\mathbb{B}^{*}(\Delta)=\mathbb{B}(\Delta) \cup\left\{b_{1}, \ldots, b_{k}\right\},
$$

we then define $\bar{\mu}_{\Delta \text {,maxwir }}^{\mathrm{GRC}}$ as the maxwired GRC measure on the graph $\left(\bar{\Delta}, \mathbb{B}^{*}(\Delta)\right)$, where as before $\bar{\Delta}=\Delta \cup \partial \Delta$. With this definition we get

$$
\begin{aligned}
\mu_{\Delta, \text { maxwir }}^{\mathrm{GRC}}(\cdot) & =\bar{\mu}_{\Delta, \text { maxwir }}^{\mathrm{GRC}}\left(\cdot \mid \eta_{b}=0 \forall b \in \mathbb{B}^{*}(\Delta) \backslash \mathbb{B}(\Delta)\right) \\
& \leq \bar{\mu}_{\Delta, \text { maxwir }}^{\mathrm{GRC}}\left(\cdot \mid \eta_{b}=1 \forall b \in \mathbb{B}^{*}(\Delta) \backslash \mathbb{B}(\Delta)\right) \\
& \mathrm{F} \overline{\mathrm{K} G} \\
& \leq \bar{\mu}_{\Delta, \text { maxwir }}^{\mathrm{GRC}}\left(\cdot \mid \eta_{b}=1 \forall b \in\left(\mathbb{B}^{*}(\Delta) \backslash \mathbb{B}(\Delta)\right) \cup \mathbb{B}(\Lambda)^{\mathrm{c}}\right) \\
\mathrm{FKG} & =\mu_{\Lambda, \text { maxwir }}^{\mathrm{GRC}}(\cdot),
\end{aligned}
$$

proving the desired inequality (4.15). Here the first inequality uses that the strong FKG measures, conditioned on taking a fixed configuration $\boldsymbol{\eta}_{A}$ in a set $A$, are FKG increasing in $\boldsymbol{\eta}_{A}$, while the second inequality follows by the FKG property of $\bar{\mu}_{\Delta, \text { maxwir }}^{\mathrm{GRC}}$. 
Proof of Theorem 3.1(ii). As a consequence of (4.14) and (4.15), the net ( $\mu_{\Lambda \text {,free }}^{\mathrm{GRC}}$ ) (resp. $\left.\left(\mu_{\Lambda, \text { maxwir }}^{\mathrm{GRC}}\right)\right)$ increases (resp. decreases) as $\Lambda$ increases (in the order defined by the set inclusion), yielding the existence of the desired limits as well as their translation invariance for all monotone quasilocal functions. Since the latter generate all quasilocal functions, the claim is established.

Proof of Theorem 3.1(iii). We first prove that for any finite set of bonds $\mathbb{B}$, the measure $\mu_{\mathbb{B}}^{\mathrm{GRC}}\left(\boldsymbol{\eta}_{\mathbb{B}} \mid \boldsymbol{\eta}_{\mathbb{B}^{\mathrm{c}}}\right)$ is strong FKG. To this end, we express $\mu_{\mathbb{B}}^{\mathrm{GRC}}\left(\boldsymbol{\eta}_{\mathbb{B}} \mid \boldsymbol{\eta}_{\mathbb{B}^{\mathrm{c}}}\right)$ as a limit of finite volume measures which can be expressed as conditionals of the finite volume measures $\mu_{\Delta \text {,free }}^{\text {GRC }}$. Using that $\mu_{\Delta \text {,free }}^{\mathrm{GRC}}$ is strong FKG we then will conclude that $\mu_{\mathbb{B}}^{\mathrm{GRC}}\left(\boldsymbol{\eta}_{\mathbb{B}} \mid \boldsymbol{\eta}_{\mathbb{B}^{\mathrm{c}}}\right)$ is strong FKG.

Let $\Delta$ be a finite subset of $\mathbb{Z}^{d}$, let $\boldsymbol{\eta} \in\{0,1\}^{\mathbb{B}_{0}\left(\mathbb{Z}^{d}\right)}$ and let

$$
\eta_{b}^{(\Delta)}= \begin{cases}\eta_{b} & b \in \mathbb{B}_{0}(\Delta) \\ 0 & \text { otherwise. }\end{cases}
$$

Then we have $W_{\mathbb{B}_{0}(\Delta)}^{\mathrm{GRC}}\left(\boldsymbol{\eta}_{\mathbb{B}_{0}(\Delta)}^{(\Delta)} \mid \boldsymbol{\eta}_{\mathbb{B}_{0}(\Delta)}^{(\Delta)}\right)=e^{-\beta h_{\max }|\Delta|} W_{\Delta \text {,free }}^{\mathrm{GRC}}\left(\boldsymbol{\eta}^{(\Delta)}\right)$. Consequently,

$$
\mu_{\mathbb{B}_{0}(\Delta)}^{\mathrm{GRC}}\left(\cdot \mid \boldsymbol{\eta}_{\mathbb{B}_{0}(\Delta)^{\mathrm{c}}}^{(\Delta)}\right)=\mu_{\Delta, \text { free }}^{\mathrm{GRC}}\left(\cdot \mid \boldsymbol{\eta}_{\mathbb{B}_{0}(\Delta)^{\mathrm{c}}}^{(\Delta)}\right) .
$$

Since the latter measure is strong FKG and since $\left(\mu_{\mathbb{B}}^{\mathrm{GRC}}\right)$ form a consistent family of specifications, $\mu_{\mathbb{B}}^{\mathrm{GRC}}\left(\cdot \mid \boldsymbol{\eta}_{\mathbb{B} c}^{(\Delta)}\right)$ is strong FKG as well for any $\mathbb{B} \subset \mathbb{B}(\Delta)$ (use that conditioned strong FKG measures are still strong FKG). The strong FKG property of the measure $\mu_{\mathbb{B}}^{\mathrm{GRC}}\left(\boldsymbol{\eta}_{\mathbb{B}} \mid \boldsymbol{\eta}_{\mathbb{B}}\right)$ now follows from the fact that $\mu_{\mathbb{B}}^{\mathrm{GRC}}\left(\cdot \mid \boldsymbol{\eta}_{\mathbb{B}^{\mathrm{c}}}^{(\Delta)}\right) \rightarrow \mu_{\mathbb{B}}^{\mathrm{GRC}}\left(\cdot \mid \boldsymbol{\eta}_{\mathbb{B}^{c}}\right)$ as $\Delta \nearrow \mathbb{Z}^{d}$, which in turn is a consequence of the observation that for each $\boldsymbol{\eta}$ there is a finite $\Delta$ such that the number of components of the graph $\left(\Delta, \mathbb{B}_{\text {occ }}\left(\eta^{(\Delta)}\right)\right)$ that reach from $\mathbb{V}(\mathbb{B})$ to the boundary of $\Delta$ is equal to the number of infinite components of $\left(\mathbb{Z}^{d}, \mathbb{B}_{0}(\eta)\right)$ that touch $\mathbb{V}(\mathbb{B})$. (Here we used that there are only finitely many infinite clusters connected to $\mathbb{B}$.)

Hence $\mu_{\mathbb{B}}^{\mathrm{GRC}}\left(\cdot \mid \boldsymbol{\eta}_{\mathbb{B}^{c}}\right)$ is strong FKG for all $\boldsymbol{\eta}$ and all finite sets of bonds $\mathbb{B}$. In particular, $\mu_{\mathbb{B}}^{\mathrm{GRC}}\left(\cdot \mid \boldsymbol{\eta}_{\mathbb{B}^{\mathrm{c}}}\right)$ is increasing in the boundary condition (the specifications are consistent), and

$$
\mu_{\mathbb{B}}^{\mathrm{GRC}}\left(\cdot \mid \boldsymbol{\eta}_{\mathbb{B}^{c}}^{(0)}\right) \underset{\mathrm{FKG}}{\leq} \mu_{\mathbb{B}}^{\mathrm{GRC}}\left(\cdot \mid \boldsymbol{\eta}_{\mathbb{B}^{\mathrm{c}}}\right) \underset{\mathrm{FKG}}{\leq} \mu_{\mathbb{B}}^{\mathrm{GRC}}\left(\cdot \mid \boldsymbol{\eta}_{\mathbb{B}^{\mathrm{c}}}^{(1)}\right),
$$

where $\boldsymbol{\eta}^{(i)}$ is the configuration with $\boldsymbol{\eta}^{(i)}=i$ for all $b \in \mathbb{B}_{0}\left(\mathbb{Z}^{d}\right)$. Choosing $\mathbb{B}=\mathbb{B}(\Lambda)$ and continuing by further conditioning as in the proof of (4.14) and (4.20), we get

$$
\mu_{\Lambda, \text { free }}^{\mathrm{GRC}}(\cdot) \underset{\mathrm{FK} \mathrm{K}}{\leq} \mu_{\mathbb{B}(\Lambda)}^{\mathrm{GRC}}\left(\cdot \mid \boldsymbol{\eta}_{\mathbb{B}(\Lambda)^{\mathrm{c}}}\right) \underset{\mathrm{FK} \mathrm{K} G}{\leq} \mu_{\Lambda, \text { maxwir }}^{\mathrm{GRC}}(\cdot) .
$$

If $\mu$ is a Gibbs measure, the bound (4.24) and the DLR equation (2.12) imply that

$$
\mu_{\Lambda, \text { free }}^{\mathrm{GRC}}(\cdot) \underset{\mathrm{FKG}}{\leq} \mu^{\mathrm{GRC}}(\cdot) \underset{\mathrm{F} \mathrm{K} \mathrm{G}}{\leq} \mu_{\Lambda, \text { maxwir }}^{\mathrm{GRC}}(\cdot) .
$$

Taking the limit $\Lambda \nearrow \mathbb{Z}^{d}$, we get statement (iii) for an arbitrary GRC Gibbs state $\mu$.

In order to prove statement (iii) for a GRC limit state, we use that for any sequence of finite sets $\mathbb{B}_{n}$ with $\mathbb{B}_{n} \nearrow \mathbb{B}_{0}\left(\mathbb{Z}^{d}\right)$, we can find a sequence $\Lambda_{n}$ of finite subsets in $\mathbb{Z}^{d}$ such that $\Lambda_{n} \nearrow \mathbb{Z}^{d}$ and $\mathbb{B}\left(\Lambda_{n}\right) \subset \mathbb{B}_{n}$. Given such a sequence and a sequence of boundary condition $\boldsymbol{\eta}^{(n)}$, we then proceed as above to bound

$$
\mu_{\Lambda_{n}, \text { free }}^{\mathrm{GRC}}(\cdot) \underset{\mathrm{FKG}}{\leq} \mu_{\mathbb{B}_{n}}^{\mathrm{GRC}}\left(\cdot \mid \boldsymbol{\eta}_{\mathbb{B}_{n}(\Lambda)^{\mathrm{c}}}^{(n)}\right) \underset{\mathrm{FKG}}{\leq} \mu_{\Lambda_{n}, \text { maxwir }}^{\mathrm{GRC}}(\cdot) .
$$


Taking the limit $n \rightarrow \infty$, this proves statement (iii) for an arbitrary RC limit state.

Proof of Theorem 3.1(iv). Let $g$ be a monotone increasing function, depending only on bonds $\mathbb{B}_{0}(\Delta)$ for some finite $\Delta$. For each finite $\Lambda \subset \mathbb{Z}^{d}$ define

$$
g_{\Lambda}=\sum_{x: \tau^{x}(\Delta) \subset \Lambda} g \circ \tau^{x}
$$

where $\tau$ is the shift operator. Let $\mu_{\Lambda \text {,free }}^{\mathrm{GRC}, J_{1}, \alpha}$ and $\mu_{\Lambda, \text { maxwir }}^{\mathrm{GRC}, J_{1}, \alpha}$ be the GRC measures with free and maxwired boundary condition and coupling $J=J_{1}$, however, with the weights in (3.1) and (3.4) multiplied by the function $e^{\alpha g_{\Lambda}}$. We then consider the generating function

$$
Z_{\Lambda, \text { free }}^{(\alpha)}=\sum_{\boldsymbol{\eta} \in \mathbb{B}_{0}\left(\mathbb{Z}^{d}\right)} e^{\alpha g_{\Lambda}(\boldsymbol{\eta})}\left(e^{\beta J}-1\right)^{|\boldsymbol{\eta}|} \prod_{C(\boldsymbol{\eta})} \Theta_{\text {free }}(C(\boldsymbol{\eta})),
$$

where, as in formula (3.1), the product runs over all connected components $C(\boldsymbol{\eta})$ of the graph $\left(\Lambda, \mathbb{B}_{\text {occ }}(\boldsymbol{\eta}) \cap \mathbb{B}_{0}(\Lambda)\right)$. Similarly, we introduce the generating function $Z_{\Lambda \text {,maxwir }}^{(\alpha)}$ for the moments of $g_{\Lambda}$ with respect to $\mu_{\Lambda \text {,maxwir }}^{\mathrm{GRC}}$. Consider now a volume $\Lambda$ that is a disjoint union of two volumes $\Lambda_{1}$ and $\Lambda_{2}$. Then we have the following submultiplicative bound

$$
Z_{\Lambda, \text { free }}^{(\alpha)} \geq Z_{\Lambda_{1}, \text { free }}^{(\alpha)} Z_{\Lambda_{2}, \text { free }}^{(\alpha)} e^{O\left(\alpha\left|\mathbb{B}\left(\Lambda_{1}\right) \cap \mathbb{B}\left(\Lambda_{2}\right)\right|\right)},
$$

which can be easily obtained by restricting the sum in (4.28) to those $\boldsymbol{\eta}$ which are zero on the bonds in $\mathbb{B}\left(\Lambda_{1}\right) \cap \mathbb{B}\left(\Lambda_{2}\right)$, and observing that

$$
g_{\Lambda}=g_{\Lambda_{1}}+g_{\Lambda_{2}}+\sum_{\substack{x: \tau^{x}(\Delta) \subset \Lambda, \tau^{x}(\Delta) \cap \Lambda_{1} \neq \emptyset, \tau^{x}(\Delta) \cap \Lambda_{2} \neq \emptyset}} g \circ \tau^{x} .
$$

By standard subadditivity arguments, it follows from (4.29) that the "free energy"

$$
f(\alpha)=\lim _{\Lambda \nearrow \mathbb{Z}^{d}} \frac{1}{|\Lambda|} \log Z_{\Lambda, \text { free }}^{(\alpha)}
$$

exists and is convex in $\alpha$. In (4.31), we assume that the limit is taken over cubes of the form $\Lambda_{n}=\{-n, \ldots, n\}^{d}$.

The same limit is obtained if $Z_{\Lambda \text {,free }}^{(\alpha)}$ is replaced by $Z_{\Lambda \text {,maxwir }}^{(\alpha)}$. Indeed, observing that $Z_{\Lambda \text {,maxwir }}^{(\alpha)}$ can be bounded from below by restricting the sum over configurations to those for which $\boldsymbol{\eta}$ is 0 on $\mathbb{B}(\Lambda) \backslash \mathbb{B}_{0}(\Lambda)$, we get

$$
Z_{\Lambda, \text { maxwir }}^{(\alpha)} \geq e^{O(\alpha|\partial \Lambda|)} Z_{\Lambda, \text { free }}^{(\alpha)} \prod_{x \in \partial \Lambda} \Theta_{\Lambda, m}(\{x\})
$$

provided $m \in Q_{\max }$. To get an upper bound on $Z_{\Lambda \text {,maxwir }}^{(\alpha)}$, observe that $\Theta_{\Lambda, m} \leq \Theta_{\text {free }}$ by our assumption (3.3). As a consequence,

$$
Z_{\Lambda, \text { maxwir }}^{(\alpha)} \leq e^{O(\alpha|\partial \Lambda|)} Z_{\bar{\Lambda}, \text { free }}^{(\alpha)}
$$

where, as before, $\bar{\Lambda}=\Lambda \cup \partial \Lambda$. While $\bar{\Lambda}$ is not of the form $\{-n, \ldots, n\}^{d}$ required for the existence of the limit (4.31), it can easily bounded by a term of this form times a boundary term with the help of (4.29). We therefore have shown that $Z_{\Lambda \text {,maxwir }}^{(\alpha)}$ and $Z_{\Lambda \text {,free }}^{(\alpha)}$ give rise to the same free energy $f(\alpha)$. 
Moreover, by differentiating, we find that

$$
\limsup _{\Lambda \nearrow \mathbb{Z}^{d}} \mu_{\Lambda, \text { maxwir }}^{\mathrm{GRC}, J_{1}}\left(\frac{g_{\Lambda}}{|\Lambda|}\right) \leq \frac{\mathrm{d} f}{\mathrm{~d} \alpha^{+}}\left(\alpha_{1}\right) \leq \frac{\mathrm{d} f}{\mathrm{~d} \alpha^{-}}\left(\alpha_{2}\right) \leq \liminf _{\Lambda \nearrow \mathbb{Z}^{d}} \mu_{\Lambda, \text { free }}^{\mathrm{GRC}, J_{1}, \alpha}\left(\frac{g_{\Lambda}}{|\Lambda|}\right),
$$

where $0<\alpha_{1}<\alpha_{2}<\alpha$ are arbitrary.

Since $g_{\Lambda}$ is increasing, we have from (4.15) and the translation invariance of $\mu_{\text {maxwir }}^{\mathrm{GRC} J_{1}}$ that the left hand side of (4.34) equals $\mu_{\text {maxwir }}^{\mathrm{GRC}, J_{1}}(g)$. Thus we just need to show that if $\alpha$ is small enough then $\mu_{\Lambda \text {,free }}^{\mathrm{GRC}, J_{1}, \alpha}$ is FKG dominated by $\mu_{\Lambda \text {,free }}^{\mathrm{GRC}, J_{2}}$. To this end recall that the second measure can be directly generated by the weights $W_{\Lambda \text {,free }}^{J_{2}}$ defined in (3.1), while the first one can be generated by the weights $e^{\alpha g_{\Lambda}} W_{\Lambda, \text { free }}^{J_{1}}$. As a consequence, we have

$$
\mu_{\Lambda, \text { free }}^{\mathrm{GRC}, J_{1}, \alpha}(\cdot)=\frac{\mu_{\Lambda, \text { free }}^{\mathrm{GRC} J_{2}}\left(\cdot G_{\Lambda}\right)}{\mu_{\Lambda, \text { free }}^{\mathrm{GRC}, J_{2}}\left(G_{\Lambda}\right)},
$$

where

$$
G_{\Lambda}(\boldsymbol{\eta})=e^{\alpha g_{\Lambda}(\boldsymbol{\eta})} \frac{W_{\Lambda, \text { free }}^{J_{1}}(\boldsymbol{\eta})}{W_{\Lambda, \text { free }}^{J_{2}}(\boldsymbol{\eta})} .
$$

Hence it suffices to ensure that the function $\boldsymbol{\eta} \mapsto G_{\Lambda}(\boldsymbol{\eta})$ is monotone decreasing in $\boldsymbol{\eta}$. Let us define the variance of $g$ by the formula

$$
\operatorname{var}(g)=\sup _{\bar{b}} \sup _{\substack{\boldsymbol{\eta}, \boldsymbol{\eta}^{\prime}: \eta_{b}=\eta_{b}^{\prime} \\ \forall b \neq \bar{b}}}\left|g(\boldsymbol{\eta})-g\left(\boldsymbol{\eta}^{\prime}\right)\right| .
$$

Note that $\operatorname{var}(g)$ is the maximum amount that $g$ can change by flipping a single bond. Since

$$
\frac{W_{\Lambda, \text { free }}^{J_{1}}}{W_{\Lambda, \text { free }}^{J_{2}}}(\boldsymbol{\eta})=\left[\frac{e^{\beta J_{1}}-1}{e^{\beta J_{2}}-1}\right]^{|\boldsymbol{\eta}|},
$$

the monotonicity of $G_{\Lambda}$ is guaranteed for instance by $e^{\alpha \operatorname{var}(g)\left|\mathbb{B}_{0}(\Delta)\right|}\left(e^{\beta J_{1}}-1\right) \leq\left(e^{\beta J_{2}}-1\right)$. For $J_{1}<J_{2}$, this in turn is achieved by taking $\alpha$ small enough. Thus, for $\alpha$ sufficiently small and positive, we have

$$
\mu_{\text {maxwir }}^{\mathrm{GRC}, J_{1}}(g) \leq \liminf _{\Lambda \nearrow \mathbb{Z}^{d}} \mu_{\Lambda, \text { free }}^{\mathrm{GRC}, J_{1}, \alpha}\left(\frac{g_{\Lambda}}{|\Lambda|}\right) \leq \liminf _{\Lambda \nearrow \mathbb{Z}^{d}} \mu_{\Lambda, \text { free }}^{\mathrm{GRC}, J_{2}}\left(\frac{g_{\Lambda}}{|\Lambda|}\right) \leq \mu_{\text {free }}^{\mathrm{GRC}, J_{2}}(g),
$$

where the last inequality follows from $\mu_{\Lambda \text {,free }}^{\mathrm{GRC}, J_{2}} \leq \underset{\mathrm{K} G}{\leq} \mu_{\text {free }}^{\mathrm{GRC}, J_{2}}$ and the translation invariance of $\mu_{\text {free }}^{\text {GRC, } J_{2}}$. Since $g$ was arbitrary, (3.11) is established.

Before proving item (v) of Theorem 3.1, let us present an elementary argument showing why our definition of partial order on the external fields is the only correct one, at least provided we stipulate that it be independent of the volume, $\beta>0$, and the values of $\left(q_{m}\right)$ (however, such that the strong FKG condition is still satisfied).

Let $\Lambda=\{x, y\}$, where $x$ and $y$ are nearest neighbors, and consider the event $\left\{\eta_{b}=1\right\}$ that the bond $b=\langle x, y\rangle$ is occupied. Then

$$
\mu_{\{x, y\}, \text { free }}^{\mathrm{GRC},\left(h_{m}\right)}\left(\eta_{b}=1\right)=f\left(\frac{\|\boldsymbol{a}\|_{2}}{\|\boldsymbol{a}\|_{1}}\right),
$$


where $f(x)=x^{2} /\left(1+x^{2}\right)$ and where $\|\cdot\|_{1}$ and $\|\cdot\|_{2}$ are the $\ell^{1}$ and $\ell^{2}$ norms of the vector $\boldsymbol{a}=\left(e^{\beta h_{1}}, \ldots, e^{\beta h_{q}}\right)$ in the metric with weights $\left(q_{m}\right)$, i.e.,

$$
\|\boldsymbol{a}\|_{1}=\sum_{m=1}^{q} q_{m} e^{\beta h_{m}} \quad \text { and } \quad\|\boldsymbol{a}\|_{2}^{2}=\sum_{m=1}^{q} q_{m} e^{2 \beta h_{m}} .
$$

Since $x \mapsto f(x)$ is strictly increasing, $\mu_{\{x, y\}}^{\mathrm{GRC},\left(h_{m}\right)}$,free increases with $\left(h_{m}\right)$ if and only if $\frac{\|\boldsymbol{a}\|_{2}}{\|\boldsymbol{a}\|_{1}}$ does. If this is to hold independently of the $q_{m}$ 's, then also

$$
\lim _{\alpha q_{k}=q_{l} \rightarrow \infty} q_{k} \frac{\|\boldsymbol{a}\|_{2}^{2}}{\|\boldsymbol{a}\|_{1}^{2}}=\frac{1+\alpha e^{-2 \beta\left(h_{k}-h_{l}\right)}}{\left[1+\alpha e^{-\beta\left(h_{k}-h_{l}\right)}\right]^{2}}
$$

must be increasing for all $\alpha>0$. (In the above limit, we fix all $q_{m}$ 's with $m \neq k, l$.)

We want to show that the condition

$$
h_{k}^{\prime}-h_{l}^{\prime} \geq h_{k}-h_{l} \quad \text { whenever } \quad h_{k}-h_{l}>0
$$

is necessary for the claim (3.12). To this end, we first show that the condition

$$
h_{k}^{\prime}-h_{l}^{\prime} \geq 0 \quad \text { whenever } \quad h_{k}-h_{l}>0
$$

is necessary for (3.12) to hold. To see this, assume $h_{k}>h_{l}$ and $h_{k}^{\prime}-h_{l}^{\prime}<0$. Then for large enough $\beta$, the r.h.s. of (4.42) is close to 1 for $\left(h_{m}\right)$ and close to $\alpha^{-1}$ for $\left(h_{m}^{\prime}\right)$. Taking $\alpha>1$, we see that the desired monotonicity of $\mu_{\{x, y\} \text {,free }}^{\mathrm{GRC},\left(h_{m}\right)}\left(\eta_{b}=1\right)$ is violated. Hence the condition (4.44) is necessary.

Now take $\alpha=1$ in (4.42). This leads to the function $x \mapsto \frac{1}{2} \cosh (x)[\cosh (x / 2)]^{-1}$, which is even and strictly increasing for $x>0$. Hence (4.42) increases under the replacement $\left(h_{m}\right) \rightarrow$ $\left(h_{m}^{\prime}\right)$ if and only if $\left|h_{k}^{\prime}-h_{l}^{\prime}\right| \geq\left|h_{k}-h_{l}\right|$, which together with (4.44) gives the necessity of (4.43).

The following argument shows that the condition (4.43) it is also sufficient.

Proof of Theorem 3.1(v). Let $\left(h_{m}\right)$ and $\left(h_{m}^{\prime}\right)$ be two sets of fields such that $\left(h_{m}\right) \preccurlyeq\left(h_{m}^{\prime}\right)$. In order to prove (3.12) and (3.13), we need to establish that the functions

$$
\boldsymbol{\eta} \mapsto \frac{W_{\Lambda, \text { free },\left(h_{m}^{\prime}\right)}^{\mathrm{GR}}(\boldsymbol{\eta})}{W_{\Lambda, \text { free }}^{\mathrm{GRC}\left(h_{m}\right)}(\boldsymbol{\eta})}=\Xi_{\text {free }}(\boldsymbol{\eta}), \quad \boldsymbol{\eta} \mapsto \frac{W_{\Lambda, m}^{\mathrm{GRC},\left(h_{m}^{\prime}\right)}(\boldsymbol{\eta})}{W_{\Lambda, m}^{\mathrm{GRC},\left(h_{m}\right)}(\boldsymbol{\eta})}=\Xi_{m}(\boldsymbol{\eta})
$$

are monotone increasing with $\boldsymbol{\eta}$ (the rest follows by (3.8), (3.9) and an inequality of (4.35)type). It suffices to study the single-bond flips. Let $b=\langle x, y\rangle$ be a nearest-neighbor bond such that $\eta_{b}=0$ and let $\boldsymbol{\eta}^{b}$ be the configuration obtained by flipping $\eta_{b}$ to 1 . There are two scenarios: (1) $x \leftrightarrow y$ in $\boldsymbol{\eta},(2) x \leftrightarrow y$ in $\boldsymbol{\eta}$.

In the case (1), $\Xi_{\text {free }}(\boldsymbol{\eta})=\Xi_{\text {free }}\left(\boldsymbol{\eta}^{b}\right)$, as follows by the inspection of (3.2), and similarly for the maxwired boundary condition. In the case (2), there are two components $A$ and $B$ in $\boldsymbol{\eta}$, each at one end of the bond $b$. By flipping $\eta_{b}$ to $1, A$ and $B$ become connected in one component that we denote by $C$. Note that $|\mathbb{V}(C)|=|\mathbb{V}(A)|+|\mathbb{V}(B)|$. Since the remaining components are not affected by this flip, it is easily seen that

$$
\frac{\Xi_{\text {free }}\left(\boldsymbol{\eta}^{b}\right)}{\Xi_{\text {free }}(\boldsymbol{\eta})}=\frac{\Theta_{\text {free }}^{\mathrm{GRC},\left(h_{m}\right)}(A) \Theta_{\text {free }}^{\mathrm{GRC},\left(h_{m}\right)}(B)}{\Theta_{\text {free },\left(h_{m}^{\prime}\right)}^{\mathrm{GRC}}(A) \Theta_{\text {free }\left(h_{m}^{\prime}\right)}^{\mathrm{GRC}}(B)} \frac{\Theta_{\text {free },\left(h_{m}^{\prime}\right)}^{\mathrm{GRC}}(C)}{\Theta_{\text {free },\left(h_{m}\right)}^{\mathrm{GR}}(C)},
$$

and similarly for the maxwired boundary condition. We are thus reduced to showing that the r.h.s. of (4.46) is no less than 1, and again similarly for maxwired. 
GIBBS STATES OF GRAPHICAL REPRESENTATIONS IN THE POTTS MODEL

We begin with the free boundary condition. Let $a_{m}, b_{m}, c_{m}$ have literally the same meaning as in (4.7) and let $a_{m}^{\prime}, b_{m}^{\prime}, c_{m}^{\prime}$ denote the corresponding quantities for $\left(h_{m}\right)$ replaced by $\left(h_{m}^{\prime}\right)$. Note that $c_{m}=a_{m} b_{m}$ and $c_{m}^{\prime}=a_{m}^{\prime} b_{m}^{\prime}$. Then the condition that the r.h.s. of (4.46) be no less than 1 reads

$$
\left(\sum_{j=1}^{q} q_{j} a_{j}^{\prime}\right)\left(\sum_{k=1}^{q} q_{k} b_{k}^{\prime}\right)\left(\sum_{l=1}^{q} q_{l} a_{l} b_{l}\right) \leq\left(\sum_{j=1}^{q} q_{j} a_{j}\right)\left(\sum_{k=1}^{q} q_{k} b_{k}\right)\left(\sum_{l=1}^{q} q_{l} a_{l}^{\prime} b_{l}^{\prime}\right) .
$$

We will prove this in two steps; first we "move" the prime from $a_{j}$ 's in the first bracket on the 1.h.s. to the ones in the last bracket and then do the same with the prime over $b_{k}$ in the second bracket on the left. Consider the identity

$$
a_{j}^{\prime} a_{l} b_{l}+a_{l}^{\prime} a_{j} b_{j}=\frac{1}{2}\left(a_{j}^{\prime} a_{l}+a_{j} a_{l}^{\prime}\right)\left(b_{l}+b_{j}\right)+\frac{1}{2}\left(a_{j}^{\prime} a_{l}-a_{j} a_{l}^{\prime}\right)\left(b_{l}-b_{j}\right) .
$$

Observing that $\left(h_{m}\right) \preccurlyeq\left(h_{m}^{\prime}\right)$ implies

$$
\left(a_{j}^{\prime} a_{l}-a_{j} a_{l}^{\prime}\right)\left(b_{l}-b_{j}\right) \leq 0 \leq\left(a_{j}^{\prime} a_{l}-a_{j} a_{l}^{\prime}\right)\left(b_{j}-b_{l}\right),
$$

we can bound the r.h.s. of (4.48) by interchanging $b_{l}$ and $b_{j}$. This allows us to conclude that

$$
\text { 1.h.s. of }(4.47) \leq\left(\sum_{j=1}^{q} q_{j} a_{j}\right)\left(\sum_{k=1}^{q} q_{k} b_{k}^{\prime}\right)\left(\sum_{l=1}^{q} q_{l} a_{l}^{\prime} b_{l}\right) \text {. }
$$

In order to perform the same trick on $b_{k}^{\prime}$, which will lead to the desired formula (4.47), we will need that $h_{k}^{\prime}-h_{l}^{\prime}>0$ implies $h_{k}^{\prime}-h_{l}^{\prime} \geq h_{k}-h_{l}$. After a moment's thought, the latter is a trivial consequence of our assumption (3.7).

In the case of maxwired boundary condition, let both measures be defined using the same boundary "value" $m$ with $h_{m}=h_{\max }$ and $h_{m}^{\prime}=h_{\max }^{\prime}$ (such a choice always exists, due to $\left.\left(h_{m}\right) \preccurlyeq\left(h_{m}^{\prime}\right)\right)$. We need to distinguish whether any of the components $A, B$ connects to the boundary or not. If $\mathbb{V}(A) \cap \partial \Lambda=\emptyset$ and $\mathbb{V}(B) \cap \partial \Lambda=\emptyset$, we are in the same situation as for the free boundary condition. If $\mathbb{V}(A) \cap \partial \Lambda \neq \emptyset$ but $\mathbb{V}(B) \cap \partial \Lambda=\emptyset$, then we have to check the inequality

$$
a_{m}^{\prime} a_{m} b_{m}\left(\sum_{k=1}^{q} q_{k} b_{k}^{\prime}\right) \leq a_{m}^{\prime} a_{m} b_{m}^{\prime}\left(\sum_{k=1}^{q} q_{k} b_{k}\right) .
$$

This is implied by the inequality $b_{m} b_{k}^{\prime} \leq b_{m}^{\prime} b_{k}$, which in turn follows from the assumption $\left(h_{m}\right) \preccurlyeq\left(h_{m}^{\prime}\right)$ and the fact that $h_{m}=h_{\max }$ and $h_{m}^{\prime}=h_{\max }^{\prime}$. In the case when $\mathbb{V}(A) \cap \partial \Lambda \neq \emptyset$ and $\mathbb{V}(B) \cap \partial \Lambda \neq \emptyset$, (4.47) (modified for the $m$-wired boundary condition) is fulfilled with the equality sign.

Proof of Corollary to Theorem 3.1. Items (i) and (ii) are direct consequences of (3.10). Since $\mu_{\Lambda, \text { maxwir }}^{\mathrm{GRC}}\left(0 \leftrightarrow \Lambda^{\mathrm{c}}\right) \downarrow \mu_{\text {maxwir }}^{\mathrm{GRC}}(0 \leftrightarrow \infty)$ by Lemma 4.1 , the claim (iii) follows from the fact that a monotone decreasing sequence of monotone increasing continuous functions (of parameter $J$ in our case) has a right continuous limit. To prove claims (iv) and (v), we note that the map $J \mapsto \tilde{P}(\beta, J, h)$ is non-decreasing. By (3.10) and $(3.11)$, one has $\tilde{P}(\beta, J, h) \leq P(\beta, J, h)$ for all $J$ and $\tilde{P}\left(\beta, J_{2}, h\right) \geq P\left(\beta, J_{1}, h\right)$ for all $J_{1}<J_{2}$, which implies the remaining part of (iv). Combining the monotonicity of $P(\beta, J, h)$ and $\tilde{P}(\beta, J, h)$ with the above two inequalities, we get $(\mathrm{v})$.

We close this section with an FKG domination lemma which will be used to prove Theorem 3.2 in the next section. We need some notation. First, for a finite set $\Lambda$ and any subset 
$D \subset \partial \Lambda$, where, as before, $\partial \Lambda=\left\{x \in \mathbb{Z}^{d} \mid \operatorname{dist}(x, \Lambda)=1\right\}$, we define the $D$-maxwired measure in the volume $\Lambda$ as the measure

$$
\mu_{\Lambda, D, \text { maxwir }}^{\mathrm{GRC}}(\cdot)=\mu_{\Lambda, \text { maxwir }}^{\mathrm{GRC}}\left(\cdot \mid \eta_{b}=0 \forall b \in \mathbb{B}(\Lambda) \backslash \mathbb{B}_{0}(\Lambda \cup D)\right) .
$$

Note that $\mu_{\Lambda, D \text {,maxwir }}^{\mathrm{GRC}}(\cdot)$ is identical to the free measure $\mu_{\Lambda, \text { free }}^{\mathrm{GRC}}(\cdot)$ if $D=\emptyset$ and identical to the maxwired measure $\mu_{\Lambda, \text { maxwir }}^{\text {GRC }}(\cdot)$ if $D=\partial \Lambda$.

We also generalize the $m$-wired measure $\mu_{\Lambda, m}^{\mathrm{GRC}}$. To this end we introduce, for any finite volume $\Lambda \subset \mathbb{Z}^{d}$ and any configuration $\sigma: \partial \Lambda \rightarrow\{1,2, \ldots, q\}$, a measure $\mu_{\Lambda, \sigma}^{\text {GRC }}$ that is obtained by normalizing the weight

$$
W_{\Lambda, \boldsymbol{\sigma}}^{\mathrm{GRC}}(\boldsymbol{\eta})=\left(e^{\beta J}-1\right)^{|\boldsymbol{\eta}|} \prod_{i<j} \mathbb{1}_{\left\{\partial_{i} \Lambda \leftrightarrow \partial_{j} \Lambda\right\}}(\boldsymbol{\eta}) \prod_{C(\boldsymbol{\eta})} \Theta_{\Lambda, \boldsymbol{\sigma}}(C(\boldsymbol{\eta})) .
$$

Here $\partial_{i} \Lambda$ is the set of all $x \in \partial \Lambda$ such that $\sigma_{x}=i, \partial_{i} \Lambda \leftrightarrow \partial_{j} \Lambda$ is the event that the sets $\partial_{i} \Lambda$ and $\partial_{j} \Lambda$ are not connected by a path of occupied bonds, and

$$
\Theta_{\Lambda, \sigma}(C)= \begin{cases}\Theta_{\text {free }}(C) & \mathbb{V}(C) \cap \Lambda^{\mathrm{c}}=\emptyset \\ e^{\beta h_{m}|\mathbb{V}(C)|} & \mathbb{V}(C) \cap \partial_{m} \Lambda \neq \emptyset .\end{cases}
$$

It is not hard to see that for the standard RC model (with $q_{m}=1$ for all $m=1,2, \ldots, q$ ) $\mu_{\Lambda, \boldsymbol{\sigma}}^{\mathrm{RC}}$ is in fact the RC marginal of $\mu_{\Lambda, \mathbb{B}(\Lambda)}^{\mathrm{ES}}\left(\cdot \mid \boldsymbol{\sigma}_{\Lambda^{\mathrm{c}}}, \boldsymbol{\eta}_{\mathbb{B}(\Lambda)^{\mathrm{c}}}\right)$, while $\mu_{\Lambda, D \text {,maxwir }}^{\mathrm{RC}}$ is the RC marginal of $\mu_{\Lambda, \mathbb{B}}^{\mathrm{ES}}\left(\cdot \mid \boldsymbol{\sigma}_{\Lambda^{\mathrm{c}}}, \boldsymbol{\eta}_{\mathbb{B}^{\mathrm{c}}}\right)$, provided $\mathbb{B}=\mathbb{B}_{0}(\Lambda) \cup(\mathbb{B}(\Lambda) \cap \mathbb{B}(D)), \boldsymbol{\eta}_{\mathbb{B}(\Lambda) \backslash \mathbb{B}}=0$ and $\sigma_{x}=m$ for some (x-independent) $m \in Q_{\max }$ and all $x \in D$.

The measures $\mu_{\Lambda, \boldsymbol{\sigma}}^{\mathrm{GRC}}$ and $\mu_{\Lambda, D \text {,maxwir }}^{\mathrm{GRC}}$ satisfy the following FKG bounds:

Lemma 4.2 Let $\Lambda$ be a finite set. Then for any $\boldsymbol{\sigma}$ on $\Lambda^{\mathrm{c}}$, we have

$$
\mu_{\Lambda, \boldsymbol{\sigma}}^{\mathrm{GRC}}(\cdot) \underset{\mathrm{FKG}}{\leq} \mu_{\Lambda, \operatorname{maxwir}}^{\mathrm{GRC}}(\cdot) .
$$

Moreover, let $D \subset \partial \Lambda$. Then

$$
\mu_{\Lambda, \text { free }}^{\mathrm{GRC}}(\cdot) \underset{\mathrm{FKG}}{\leq} \mu_{\Lambda, D, \text { maxwir }}^{\mathrm{GRC}}(\cdot) \underset{\mathrm{FKG}}{\leq} \mu_{\Lambda, \text { maxwir }}^{\mathrm{GRC}}(\cdot)
$$

Proof. Using the representation (4.53), it is easy to see that the measure $\mu_{\Lambda, \boldsymbol{\sigma}}^{\mathrm{GRC}}$ can be recast as

$$
\mu_{\Lambda, \sigma}^{\mathrm{GRC}}(\cdot)=\frac{\mu_{\Lambda, \text { maxwir }}^{\mathrm{GRC}}(\cdot g)}{\mu_{\Lambda, \text { maxwir }}^{\mathrm{GRC}}(g)}
$$

where

$$
g(\boldsymbol{\eta})=\prod_{i<j} \mathbb{1}_{\left\{\partial_{i} \Lambda \leftrightarrow \partial_{j} \Lambda\right\}}(\boldsymbol{\eta}) \prod_{m} \prod_{\substack{C \\ \mathbb{V}(C) \cap \dot{\partial}_{m} \Lambda \neq \emptyset}} e^{-\left(h_{\max }-h_{m}\right)|\mathbb{V}(C)|}
$$

for any $\boldsymbol{\eta} \in\{0,1\}^{\mathbb{B}(\Lambda)}$. It turns out that the function $g$ is FKG decreasing. Indeed, each indicator $\mathbb{1}_{\left\{\partial_{i} \Lambda \leftrightarrow \partial_{j} \Lambda\right\}}(\boldsymbol{\eta})$ is clearly decreasing. The same is true for the remaining factor as is seen by noting that

$$
\sum_{\substack{C: \\ \mathbb{V}(C) \cap \dot{\partial}_{m} \Lambda \neq \emptyset}}|\mathbb{V}(C)|
$$


GIBBS STATES OF GRAPHICAL REPRESENTATIONS IN THE POTTS MODEL

being equal to the number of sites connected to $\partial \Lambda_{m}$, is an increasing function of $\boldsymbol{\eta}$. Since $h_{\max } \geq h_{m}$ and since the product of non-negative decreasing functions is decreasing, the monotonicity of $g$ is established. Since $\mu_{\Lambda, \text { maxwir }}^{\mathrm{GRC}}$ is $\mathrm{FG},(4.55)$ is proved.

To prove (4.56), it is enough to observe that the right hand side of (4.52) is FKG increasing in $D$, since $\mu_{\Lambda, D \text {,maxwir }}^{\mathrm{GRC}}$ is FKG and the event $\left\{\boldsymbol{\eta}: \eta_{b}=0 \forall b \in \mathbb{B}(\Lambda) \backslash \mathbb{B}_{0}(\Lambda \cup D)\right\}$ is FKG decreasing.

\section{The COlor(S) OF the infinite Cluster(s)}

In this section we prove Theorem 3.2. Since this result uses ES measures in its very formulation, we return to the standard RC measures (with $q_{m}=1, m=1, \ldots, q$, in $(3.2)$ ) and prove the results only for them. In addition to Lemma 4.2, the second part of Theorem 3.2 requires some control of the possible values of the spins that can be assumed on the infinite clusters. To state the theorem precisely, we introduce the notation $S(\boldsymbol{\sigma}, \boldsymbol{\eta})$ for the set of possible spin values assumed on the infinite clusters in a configuration $(\boldsymbol{\sigma}, \boldsymbol{\eta})$. (Observe that since $\nu\left(\left\{(\boldsymbol{\sigma}, \boldsymbol{\eta}): \sigma_{x} \neq \sigma_{y}, \eta_{\langle x, y\rangle}=1\right\}\right)=0$ for each $\nu \in \mathcal{G}^{\mathrm{ES}}$, each connected component has a constant spin value almost surely.)

Theorem 5.1 Let $\nu \in \mathcal{G}^{\mathrm{ES}}$. Then $S \subseteq Q_{\max }(h) \nu$-almost surely.

Remark. We believe, but have not yet been able to prove, that $|S| \leq 1 \nu$-almost surely for all translation invariant $\nu \in \mathcal{G}^{\mathrm{ES}}$.

Before we prove the above theorem, let us formulate a technical lemma.

Lemma 5.2 Let $\left(a_{k}\right)_{k \geq 1}$ be a sequence of numbers such that $1 \leq a_{k} \leq C k^{n}$ for some constant $C<\infty$ and an integer $n \geq 0$. Then for each $\epsilon>0$ and any $\bar{k} \geq C(n+1)^{n} \epsilon^{-(n+1)}$

$$
a_{k} \leq \epsilon \sum_{k^{\prime} \leq k} a_{k^{\prime}}
$$

holds for at least one $k \in\{\bar{k}, \ldots,(n+1) \bar{k}\}$.

Proof. If $n=0$, the statement follows from the observation that $1 \leq a_{k} \leq C$ and $\bar{k} \geq C \epsilon^{-1}$ implies $a_{\bar{k}} \leq C \leq \epsilon \bar{k} \leq \epsilon \sum_{k^{\prime} \leq \bar{k}} a_{k^{\prime}}$, which gives (5.1) for $k=\bar{k}$. If $n \geq 1$, suppose that $a_{k}>\epsilon \sum_{k^{\prime} \leq k} a_{k^{\prime}}$ for all $k \in\{\bar{k}, \ldots,(n+1) \bar{k}\}$. Since $a_{k^{\prime}} \geq 1$, this implies $a_{k}>\epsilon \bar{k}$ for all $k \in$ $\{\bar{k}, \ldots, 2 \bar{k}\}$ and, using induction, $a_{k}>\epsilon^{\ell} \bar{k}^{\ell}$ for all $k \in\{\ell \bar{k}, \ldots,(\ell+1) \bar{k}\}$, with $\ell \in\{1, \ldots, n\}$. In particular, $a_{(n+1) \bar{k}}>\epsilon^{n+1} \bar{k}^{n+1}$. However, this is in contradiction with the assumption $a_{(n+1) \bar{k}} \leq C(n+1)^{n} \bar{k}^{n}$ whenever $\bar{k} \geq C(n+1)^{n} \epsilon^{-(n+1)}$.

Proof of Theorem 5.1. Let $m \in\{1, \ldots, q\}$ with $h_{m}<h_{\max }$ and suppose that there is $\nu \in \mathcal{G}^{\mathrm{ES}}$ with $\nu(m \in S)>0$. Since $\mathcal{G}^{\mathrm{ES}}$ as well as the event $m \in S$ are invariant w.r.t. spatial shifts, we can suppose without loss of generality that the event

$$
\Omega_{m}^{0}=\left\{(\boldsymbol{\sigma}, \boldsymbol{\eta}): \exists C(\boldsymbol{\eta}),|C(\boldsymbol{\eta})|=\infty, \mathbb{V}(C(\boldsymbol{\eta})) \ni 0, \sigma_{0}=m\right\}
$$

has positive probability under $\nu$, i.e., $\nu\left(\Omega_{m}^{0}\right)>0$. Let $\Lambda_{k}$ be the box of side length $2 k+1$ centered at the origin and, for each $(\boldsymbol{\sigma}, \boldsymbol{\eta}) \in \Omega_{m}^{0}$ and each $k \geq 1$, let $\mathbb{V}_{k}(\boldsymbol{\eta})$ be the set of sites 
in $\Lambda_{k}$ that are connected to the origin within $\mathbb{B}_{0}\left(\Lambda_{k}\right)$, and let

$$
a_{k}=a_{k}(\boldsymbol{\eta})=\left|\mathbb{V}_{k}(\boldsymbol{\eta}) \cap \partial \Lambda_{k-1}\right| \text {. }
$$

Note that $\left|\mathbb{V}_{k}(\boldsymbol{\eta})\right| \geq \sum_{k^{\prime} \leq k} a_{k^{\prime}}$ and that $1 \leq a_{k} \leq\left|\partial \Lambda_{k-1}\right| \leq 2 d(2 k+1)^{d-1} \leq 3^{d} d k^{d-1}$, where we have used that $k \geq 1$ in the final bound. Hence, by Lemma 5.2, we know that for each $\epsilon>0$ and each $\bar{k} \geq(3 \bar{d} / \epsilon)^{d}$ there is at least one $k$, with $\bar{k} \leq k \leq d \bar{k}$, such that

$$
\left|\mathbb{V}_{k}(\boldsymbol{\eta}) \cap \partial \Lambda_{k-1}\right| \leq \epsilon\left|\mathbb{V}_{k}(\boldsymbol{\eta})\right| .
$$

By (5.4) and the subadditivity of the measure, we have for $\bar{k} \geq(3 d / \epsilon)^{d}$ that

$$
\nu\left(\Omega_{m}^{0}\right) \leq \mu\left(\cup_{\bar{k} \leq k \leq d \bar{k}} \Omega_{m, k}^{0}\right) \leq \sum_{\bar{k} \leq k \leq d \bar{k}} \nu\left(\Omega_{m, k}^{0}\right),
$$

with $\Omega_{m, k}^{0}$ denoting the event

$$
\Omega_{m, k}^{0}=\left\{\begin{array}{ll} 
& \sigma_{0}=m, 0 \leftrightarrow \partial \Lambda_{k-1}, \\
(\boldsymbol{\sigma}, \boldsymbol{\eta}): & \left|\left\{x \in \partial \Lambda_{k-1}: x \underset{\mathbb{B}_{0}\left(\Lambda_{k}\right)}{\longleftrightarrow} 0\right\}\right| \leq \epsilon\left|\left\{x \in \Lambda_{k}: x_{\mathbb{B}_{0}\left(\Lambda_{k}\right)}^{\longleftrightarrow} 0\right\}\right|
\end{array}\right\} .
$$

Here $x \underset{\mathbb{B}_{0}\left(\Lambda_{k}\right)}{\longleftrightarrow} 0$ indicates that the connection occurs within $\mathbb{B}_{0}\left(\Lambda_{k}\right)$. As a result, for each $\epsilon>0$ there is a deterministic set $\mathbb{N}_{\epsilon} \subset \mathbb{N},\left|\mathbb{N}_{\epsilon}\right|=\infty$, such that for any $k \in \mathbb{N}_{\epsilon}$ one has

$$
\nu\left(\Omega_{m, k}^{0}\right) \geq \frac{1}{d k} \nu\left(\Omega_{m}^{0}\right),
$$

by the pigeon hole principle as applied to $(5.5)$.

On the other hand, since $\Omega_{m, k}^{0}$ is a $\left(\Lambda_{k}, \mathbb{B}_{0}\left(\Lambda_{k}\right)\right)$-cylinder event, we can estimate $\nu\left(\Omega_{m, k}^{0}\right)$ using the DLR equations (2.6). Recall that $\mu_{\Lambda_{k}, \boldsymbol{\sigma}}^{\mathrm{ES}}$ is the specification (2.4) with the special choice $\Lambda=\Lambda_{k}$ and $\mathbb{B}=\mathbb{B}\left(\Lambda_{k}\right)$ and the spin boundary condition $\boldsymbol{\sigma}$ (the $\boldsymbol{\eta}$ boundary condition is irrelevant in this case). Then (2.6) reads

$$
\nu\left(\Omega_{m, k}^{0}\right)=\int \nu(\mathrm{d} \boldsymbol{\sigma}, \mathrm{d} \boldsymbol{\eta}) \mu_{\Lambda_{k}, \boldsymbol{\sigma}}^{\mathrm{ES}}\left(\Omega_{m, k}^{0}\right) .
$$

Fix $\epsilon>0$ such that $d J \epsilon+h_{m}<h_{\max }$ and pick $\widetilde{m}$ with $h_{\widetilde{m}}=h_{\max }$. Then we claim that for any $\sigma$

$$
\begin{aligned}
\mu_{\Lambda_{k}, \boldsymbol{\sigma}}^{\mathrm{ES}}\left(\Omega_{m, k}^{0}\right) & \leq \mu_{\Lambda_{k}, \boldsymbol{\sigma}}^{\mathrm{ES}}\left(\mathbb{1}_{\Omega_{m, k}^{0}} \prod_{\substack{\langle x, y\rangle: x \in \Lambda_{k}^{\mathrm{c}} \\
y \in \mathbb{V}_{k}}} e^{\beta J} \mathbb{1}_{\left\{\eta_{\langle x, y\rangle}=0\right\}}\right) \\
& =\mu_{\Lambda_{k}, \boldsymbol{\sigma}}^{\mathrm{ES}}\left(\mathbb{1}_{\Omega_{\tilde{m}, k}^{0}} e^{-\beta\left(h_{\max }-h_{m}\right)\left|\mathbb{V}_{k}\right|} \prod_{\substack{\langle x, y\rangle: x \in \Lambda_{k}^{\mathrm{c}} \\
y \in \mathbb{V}_{k}}} e^{\beta J} \mathbb{1}_{\left\{\eta_{\langle x, y\rangle}=0\right\}}\right) \\
& \leq \mu_{\Lambda_{k}, \boldsymbol{\sigma}}^{\mathrm{ES}}\left(\mathbb{1}_{\Omega_{\tilde{m}, k}^{0}} e^{-\beta\left(h_{\max }-h_{m}-d J \epsilon\right)\left|\mathbb{V}_{k}\right|}\right) \leq e^{-\beta\left(h_{\max }-h_{m}-d J \epsilon\right) k}
\end{aligned}
$$

Here, in the first step we inserted the factor $e^{\beta J}$ in order to convert an arbitrary configuration at the boundary bonds of the set $\mathbb{V}_{k}$ to the vacant bond state. More explicitly, we used the 
following estimate

$$
\begin{aligned}
& \sum_{\eta_{\langle x, y\rangle}=0,1}\left(\mathbb{1}_{\left\{\eta_{\langle x, y\rangle}=0\right\}}+\left(e^{\beta J}-1\right) \delta_{\sigma_{x}, \sigma_{y}} \mathbb{1}_{\left\{\eta_{\langle x, y\rangle}=1\right\}}\right) \\
&=\left(e^{\beta J}-1\right) \delta_{\sigma_{x}, \sigma_{y}}+1 \leq e^{\beta J}=\sum_{\eta_{\langle x, y\rangle}=0,1} e^{\beta J} \mathbb{1}_{\left\{\eta_{\langle x, y\rangle}=0\right\}} \\
&=\sum_{\eta_{\langle x, y\rangle}=0,1} e^{\beta J} \mathbb{1}_{\left\{\eta_{\langle x, y\rangle}=0\right\}}\left(\mathbb{1}_{\left\{\eta_{\langle x, y\rangle}=0\right\}}+\left(e^{\beta J}-1\right) \delta_{\sigma_{x}, \sigma_{y}} \mathbb{1}_{\left\{\eta_{\langle x, y\rangle}=1\right\}}\right)
\end{aligned}
$$

at every boundary bond. Note that there is an unconstrained summation over the bond configuration because $\Omega_{\widetilde{m}, k}^{0}$ does not depend on these boundary bonds. The conversion of an arbitrary configuration at the boundary bonds of the set $\mathbb{V}_{k}$ to the vacant bond state then allows us to flip $\sigma_{x}$ at each $x \in \mathbb{V}_{k}$ from $m$ to $\tilde{m}$, resulting in the exponential factor in the second line of (5.8). The proof of the claim (5.8) is finished by noting that, on $\Omega_{\widetilde{m}, k}^{0}$, the number of flipped bonds does not exceed $d\left|\mathbb{V}_{k} \cap \partial \Lambda_{k-1}\right| \leq d \epsilon\left|\mathbb{V}_{k}\right|$ and that $\left|\mathbb{V}_{k}\right| \geq k$.

By putting (5.6), (5.7) and (5.8) together, we get that

$$
\frac{1}{d k} \nu\left(\Omega_{m}^{0}\right) \leq \nu\left(\Omega_{m, k}^{0}\right) \leq e^{-\beta\left(h_{\max }-h_{m}-d J \epsilon\right) k} \quad \forall k \in \mathbb{N}_{\epsilon}
$$

However, since $\left|\mathbb{N}_{\epsilon}\right|=\infty$ and $k$ can be arbitrarily large, this leads to a contradiction whenever $\nu\left(\Omega_{m}^{0}\right)>0$. Hence, no such $m$ with $h_{m}<h_{\max }$ can exist and $S \subseteq\left\{m: h_{m}=h_{\max }\right\} \nu$-almost surely.

Proof of Theorem 3.2. Let us consider an ES Gibbs measure $\nu$ and use $\mu$ to denote its $\boldsymbol{\eta}$ marginal. Applying the DLR equations (2.6) for $\nu$, we get

$$
\begin{aligned}
\mu(f)=\nu(f)=\int \nu(\mathrm{d} \boldsymbol{\sigma}, \mathrm{d} \boldsymbol{\eta}) \mu_{\Lambda, \mathbb{B}(\Lambda)}^{\mathrm{ES}}\left(f \mid \boldsymbol{\sigma}_{\Lambda^{\mathrm{c}}},\right. & \left.\boldsymbol{\eta}_{\mathbb{B}(\Lambda)^{\mathrm{c}}}\right)=\int \nu(\mathrm{d} \boldsymbol{\sigma}, \mathrm{d} \boldsymbol{\eta}) \mu_{\Lambda, \boldsymbol{\sigma}}^{\mathrm{RC}}(f) \\
& \leq \int \nu(\mathrm{d} \boldsymbol{\sigma}, \mathrm{d} \boldsymbol{\eta}) \mu_{\Lambda, \text { maxwir }}^{\mathrm{RC}}(f)=\mu_{\Lambda, \text { maxwir }}^{\mathrm{RC}}(f)
\end{aligned}
$$

for any increasing cylinder function $f(\boldsymbol{\eta})$ supported on $\widetilde{\mathbb{B}} \subset \mathbb{B}(\Lambda)$. Here, the inequality follows by (4.55). Applying now (2.17), we get (3.14).

In order to prove (3.15), we have to work a bit harder. Let $\left(\Delta_{n}\right)_{n \geq 1}$ be an increasing sequence of boxes centered at the origin and let

$$
\begin{gathered}
\Lambda_{n}(\boldsymbol{\eta})=\left\{x \in \Delta_{n}: x \leftrightarrow \Delta_{n}^{\mathrm{c}}\right\} \cup\left\{x \in \Delta_{n}: x \leftrightarrow \infty\right\}, \\
D_{n}(\boldsymbol{\eta})=\partial \Lambda_{n}(\boldsymbol{\eta}) \cap\{x \leftrightarrow \infty\},
\end{gathered}
$$

and

$$
D_{n}^{\mathrm{ext}}(\boldsymbol{\eta})=\partial \Lambda_{n}(\boldsymbol{\eta}) \cap\left\{x \underset{\mathbb{B}\left(\Delta_{n}\right)^{\mathrm{c}}}{\longrightarrow} \infty\right\}
$$

Observe that $D_{n}(\boldsymbol{\eta}) \subset \partial \Delta_{n}$.

Given $\bar{\Lambda}_{n} \subset \Delta_{n}, \bar{D}_{n} \subset \partial \bar{\Lambda}_{n} \cap \partial \Delta_{n}$ and $\bar{D}_{n}^{\text {ext }} \subset \bar{D}_{n}$, we will want condition on the event

$$
\mathcal{E}_{n}=\left\{\Lambda_{n}(\boldsymbol{\eta})=\bar{\Lambda}_{n}\right\} \cap\left\{D_{n}(\boldsymbol{\eta})=\bar{D}_{n}\right\} \cap\left\{D_{n}^{\text {ext }}(\boldsymbol{\eta})=\bar{D}_{n}^{\text {ext }}\right\},
$$

using the DLR condition $(2.7)$ in $\left(\bar{\Lambda}_{n}, \overline{\mathbb{B}}_{n}\right)$, where

$$
\overline{\mathbb{B}}_{n}=\mathbb{B}_{0}\left(\bar{\Lambda}_{n}\right) \cup\left(\mathbb{B}\left(\bar{\Lambda}_{n}\right) \cap \mathbb{B}\left(\bar{D}_{n}\right)\right) .
$$


To this end, we write the event $\mathcal{E}_{n}$ as the intersection of four events: the event

$$
\mathcal{E}_{n}^{\text {int }}=\left\{x \underset{\overline{\mathbb{B}}_{n}}{\longleftrightarrow} \bar{D}_{n}^{\text {ext }} \forall x \in \bar{D}_{n} \backslash \bar{D}_{n}^{\text {ext }}\right\}
$$

which depends only on the configuration in $\overline{\mathbb{B}}_{n}$, and the events

$$
\begin{aligned}
& \mathcal{E}_{n}^{(1)}=\left\{\bar{D}_{n}^{\text {ext }}=\left\{x \in \partial \bar{\Lambda}_{n}: x \underset{\overline{\mathbb{B}}\left(\Delta_{n}\right)^{\mathrm{c}}}{\longleftrightarrow} \infty\right\}\right\}, \\
& \mathcal{E}_{n}^{(2)}=\left\{\eta_{\langle x, y\rangle}=0 \forall\langle x, y\rangle \in \mathbb{B}\left(\bar{\Lambda}_{n}\right) \backslash \overline{\mathbb{B}}_{n}\right\},
\end{aligned}
$$

and

$$
\mathcal{E}_{n}^{(3)}=\left\{x \leftrightarrow \bar{\Lambda}_{n}^{\mathrm{c}} \forall x \in \Delta_{n} \backslash \bar{\Lambda}_{n}\right\} \cap\left\{x \leftrightarrow \bar{D}_{n} \text { and } x \underset{\overline{\mathbb{B}}_{n}^{c}}{\stackrel{\leftrightarrow}{a}} \infty \forall x \in \Delta_{n} \backslash \bar{\Lambda}_{n}\right\},
$$

which depend only on the bonds in $\mathbb{B}_{n}^{\mathrm{c}}$. To see that $\mathcal{E}_{n}$ is actually the intersection of these events, we first observe that $\mathcal{E}_{n}=\left\{\Lambda_{n}(\boldsymbol{\eta})=\bar{\Lambda}_{n}\right\} \cap \mathcal{E}_{n}^{(1)} \cap \mathcal{E}_{n}^{\text {int }}$. Also, if $\mathcal{E}_{n}^{(1)} \cap \mathcal{E}_{n}^{\text {int }}$ holds, then $\left\{\Lambda_{n}(\boldsymbol{\eta})=\bar{\Lambda}_{n}\right\}$ clearly implies $\mathcal{E}_{n}^{(2)} \cap \mathcal{E}_{n}^{(3)}$. So we have to show that $\mathcal{E}_{n}^{(2)} \cap \mathcal{E}_{n}^{(3)}$ together with $\mathcal{E}_{n}^{(1)} \cap \mathcal{E}_{n}^{\text {int }}$ implies $\left\{\Lambda_{n}(\boldsymbol{\eta}) \supseteq \bar{\Lambda}_{n}\right\}$ and $\left\{\Lambda_{n}(\boldsymbol{\eta}) \subseteq \bar{\Lambda}_{n}\right\}$. The former is obvious, since the event $\mathcal{E}_{n}^{(2)}$ ensures that all points in $\bar{\Lambda}_{n}$ that are connected to $\Delta_{n}^{\mathrm{c}}$ are actually connected to $\bar{D}_{n}$, and hence to infinity. The latter follows by observing that $\mathcal{E}_{n}^{(3)}$ implies that all $x \in \Delta_{n} \backslash \bar{\Lambda}_{n}$ are connected to the complement of $\Delta_{n}$, but are not connected to infinity.

Let $f$ be a non-negative $\mathrm{FKG}$ increasing $\mathbb{B}_{0}(\Delta)$-cylinder function, where $\Delta$ is a finite set. By the assumption on $\mu$, either $q_{0}=1$ or there is at most one infinite cluster. In both cases, the spin on the infinite component(s) is uniquely defined: $\sigma_{x}=m$ with $h_{m}=h_{\max }$ for all $x$ in $\bar{D}_{n}$. Since the indicator function of the event $\mathcal{E}_{n}^{\text {int }}$ depends only on the configuration $\boldsymbol{\eta}_{\overline{\mathbb{B}}_{n}}$, while the indicator function of the event

$$
\mathcal{E}_{n}^{\mathrm{ext}}=\mathcal{E}_{n}^{(1)} \cap \mathcal{E}_{n}^{(2)} \cap \mathcal{E}_{n}^{(3)}
$$

depends only on the configuration $\boldsymbol{\eta}_{\overline{\mathbb{B}}_{n}^{c}}$, we may now use the fact the conditional expectations of the ES Gibbs measure $\nu$ are given by (2.4) to write

$$
\begin{aligned}
& \mu(f)=\nu(f) \geq \nu\left(f \mathbb{1}_{\left\{\Lambda_{n}(\cdot) \supseteq \Delta\right\}}\right) \\
& =\sum_{\bar{\Lambda}_{n} \supseteq \Delta} \sum_{m \in Q_{\max }} \nu\left(f \mathbb{1}_{\left\{\Lambda_{n}(\cdot)=\bar{\Lambda}_{n}\right\}} \mathbb{1}_{\left\{D_{n}(\cdot)=\bar{D}_{n}\right\}} \mathbb{1}_{\left\{D_{n}^{\text {ext }}(\cdot)=\bar{D}_{n}^{\text {ext }}\right\}} \mathbb{1}_{\left\{\boldsymbol{\sigma}_{\bar{D}_{n}} \equiv m\right\}}\right) \\
& \bar{D}_{n} \subseteq \partial \bar{\Lambda}_{n} \cap \partial \Delta_{n}
\end{aligned}
$$

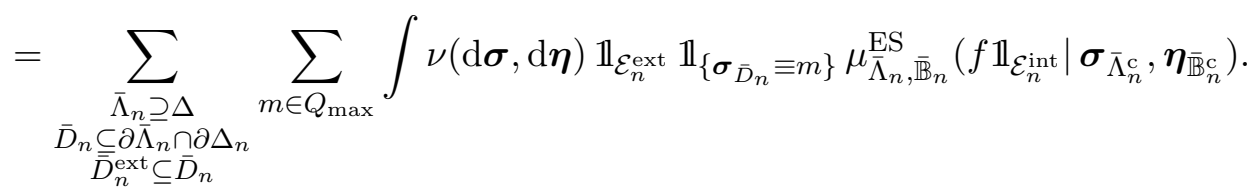

Under the condition that $\boldsymbol{\sigma}_{\bar{D}_{n}} \equiv m$, the RC marginal of $\mu_{\bar{\Lambda}_{n}, \overline{\mathbb{B}}_{n}}^{\mathrm{ES}}\left(\cdot \mid \boldsymbol{\sigma}_{\bar{\Lambda}_{n}^{c}}, \boldsymbol{\eta}_{\overline{\mathbb{B}}_{n}^{c}}\right)$ in the above equation is just the measure $\mu_{\bar{\Lambda}_{n}, \bar{D}_{n} \text {, maxwir }}^{\mathrm{RC}}$ introduced in the last section. Since the event $\mathcal{E}_{n}^{\text {int }}$ is an increasing event and since $\mu_{\bar{\Lambda}_{n}, \bar{D}_{n} \text {,maxwir }}^{\mathrm{RC}}$ is strong FKG (being given by conditioning from 
a strong FKG measure), we conclude that

$$
\begin{aligned}
\mu_{\bar{\Lambda}_{n}, \overline{\mathbb{B}}_{n}}^{\mathrm{ES}}\left(f \mathbb{1}_{\mathcal{E}_{n}^{\text {int }}} \mid \boldsymbol{\sigma}_{\bar{\Lambda}_{n}^{c}}, \boldsymbol{\eta}_{\overline{\mathbb{B}}_{n}^{c}}\right) & =\mu_{\bar{\Lambda}_{n}, \bar{D}_{n}, \text { maxwir }}^{\mathrm{RC}}\left(f \mathbb{1}_{\mathcal{E}_{n}^{\text {int }}}\right) \\
& \geq \mu_{\bar{\Lambda}_{n}, \bar{D}_{n}, \text { maxwir }}^{\mathrm{RC}}(f) \mu_{\bar{\Lambda}_{n}, \bar{D}_{n}, \text { maxwir }}^{\mathrm{RC}}\left(\mathbb{1}_{\mathcal{E}_{n}^{\text {int }}}\right) \\
& =\mu_{\bar{\Lambda}_{n}, \bar{D}_{n}, \text { maxwir }}^{\mathrm{RC}}(f) \mu_{\bar{\Lambda}_{n}, \overline{\mathbb{B}}_{n}}^{\mathrm{ES}}\left(\mathbb{1}_{\mathcal{E}_{n}^{\text {int }}} \mid \boldsymbol{\sigma}_{\bar{\Lambda}_{n}^{c}}, \boldsymbol{\eta}_{\overline{\mathbb{B}}_{n}^{c}}\right)
\end{aligned}
$$

provided $\boldsymbol{\sigma}_{\bar{D}_{n}} \equiv m$ and $\boldsymbol{\eta}_{\overline{\mathbb{B}}_{n}^{\text {c }}} \in \mathcal{E}_{n}^{\text {ext }}$. Observing finally that

$$
\mu_{\bar{\Lambda}_{n}, \bar{D}_{n}, \text { maxwir }}^{\mathrm{RC}}(f) \geq \mu_{\bar{\Lambda}_{n}, \text { free }}^{\mathrm{RC}}(f) \geq \mu_{\Delta, \text { free }}^{\mathrm{RC}}(f)
$$

by (4.56) and (4.14), we get that

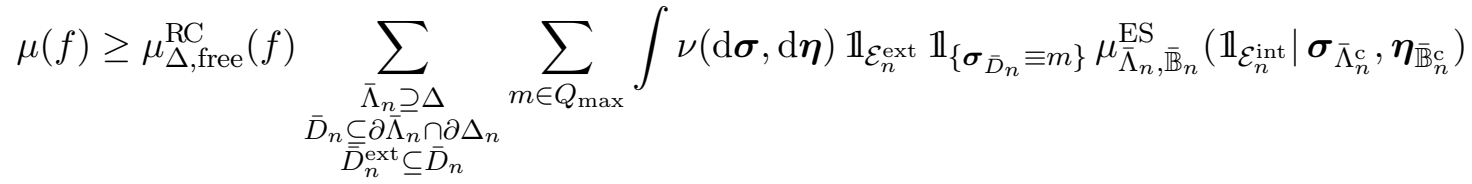

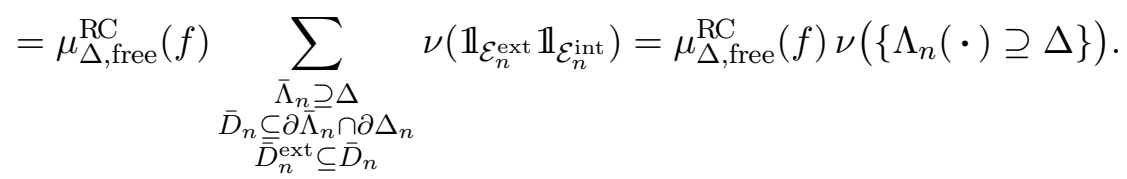

Here in the first step we used the bounds (5.22)-(5.24), in the second we used Gibbsianness of $\nu$, and in the third we used the fact that $\mathbb{1}_{\mathcal{E}_{n}^{\text {ext }}} \mathbb{1}_{\mathcal{E}_{n}^{\text {int }}}$ is the indicator function of the event (5.15) to resum over $\bar{\Lambda}_{n}, \bar{D}_{n}$ and $\bar{D}_{n}^{\text {ext }}$.

Since $\nu\left(\left\{\Lambda_{n}(\cdot) \supseteq \Delta\right\}\right)$ tends to 1 as $n \rightarrow \infty$ by the monotone convergence theorem, the proof is finished for $f \geq 0$ by taking that limit followed by $\Delta \nearrow \mathbb{Z}^{d}$. Arbitrary cylinder $f$ 's are handled by noting that $f-\min f \geq 0$.

\section{UNiqUENESS OF THE INFINITE CLUSTER}

In this section we prove that GRC Gibbs measures and weak limits of finite volume GRC measures have at most one infinite cluster almost surely (Theorem 3.3). This is a direct consequence of Theorem 1 from [13], once we show that the limiting measure satisfies the positive finite energy condition. Using a slightly stronger form of the condition than that in [13], we say that a GRC measure $\mu$ has positive finite energy if for all bonds $b \in \mathbb{B}\left(\mathbb{Z}^{d}\right)$, we have

$$
\mu\left(\eta_{b}=1 \mid \mathcal{B}_{\mathbb{B}\left(\mathbb{Z}^{d}\right) \backslash\{b\}}\right)>0 \quad \mu \text {-almost everywhere. }
$$

Here $\mathcal{B}_{\mathbb{B}\left(\mathbb{Z}^{d}\right) \backslash\{b\}}$ is the $\sigma$-algebra generated by all cylinder functions on $\{0,1\}^{\mathbb{B}\left(\mathbb{Z}^{d}\right) \backslash\{b\}}$.

We start with a lemma concerning GRC measures that are either Gibbs states or weak limit points of finite volume GRC measures.

Lemma 6.1 Let $\mu$ be a translation invariant GRC measure that is either a Gibbs state or it is a weak limit of the form $\lim _{n \rightarrow \infty} \mu_{\mathbb{B}_{n}}^{\mathrm{GRC}}\left(\cdot \mid \boldsymbol{\eta}_{n}\right)$. Then the measure $\mu$ satisfies the positive finite energy condition, provided $\beta J>0$.

Proof. Consider a finite set of bonds $\mathbb{B}$ and the characteristic function $\mathbb{1}_{\left\{\overline{\boldsymbol{\eta}}_{\mathbb{B}}\right\}}$ of the event $\left\{\boldsymbol{\eta} \mid \boldsymbol{\eta}_{\mathbb{B}}=\overline{\boldsymbol{\eta}}_{\mathbb{B}}\right\}$. The claim (6.1) will be proved once we verify that there exists a constant $c>0$ 
such that for every $\mathbb{B} \subset \mathbb{B}\left(\mathbb{Z}^{d}\right) \backslash\{b\}$ and every $\overline{\boldsymbol{\eta}}_{\mathbb{B}}$, one has

$$
\int \mu(\mathrm{d} \boldsymbol{\eta}) \mathbb{1}_{\left\{\overline{\boldsymbol{\eta}}_{\mathbb{B}}\right\}}(\boldsymbol{\eta}) \mu\left(\eta_{b}=1 \mid \mathcal{B}_{\mathbb{B}\left(\mathbb{Z}^{d}\right) \backslash\{b\}}\right)(\boldsymbol{\eta}) \geq c \int \mu(\mathrm{d} \boldsymbol{\eta}) \mathbb{1}_{\left\{\overline{\boldsymbol{\eta}}_{\mathbb{B}}\right\}}(\boldsymbol{\eta}) \mu\left(\eta_{b}=0 \mid \mathcal{B}_{\left.\mathbb{B}^{\left(\mathbb{Z}^{d}\right.}\right) \backslash\{b\}}\right)(\boldsymbol{\eta}) .
$$

Indeed, (6.2) implies that

$$
\mu\left(\eta_{b}=1 \mid \mathcal{B}_{\mathbb{B}\left(\mathbb{Z}^{d}\right) \backslash\{b\}}\right)(\boldsymbol{\eta}) \geq c \mu\left(\eta_{b}=0 \mid \mathcal{B}_{\mathbb{B}\left(\mathbb{Z}^{d}\right) \backslash\{b\}}\right)(\boldsymbol{\eta})
$$

almost surely, which in turn yields

$$
\mu\left(\eta_{b}=1 \mid \mathcal{B}_{\mathbb{B}\left(\mathbb{Z}^{d}\right) \backslash\{b\}}\right)(\boldsymbol{\eta}) \geq \frac{c}{1+c}
$$

almost surely and thus (6.1). Now, since $\mathbb{1}_{\left\{\overline{\boldsymbol{\eta}}_{\mathbb{B}}\right\}}$ is $\mathcal{B}_{\mathbb{B}\left(\mathbb{Z}^{d}\right) \backslash\{b\}}$-measurable, the inequality (6.2) is equivalent to

$$
\mu\left(\mathbb{1}_{\left\{\overline{\boldsymbol{\eta}}_{\mathbb{B}}\right\}} \mathbb{1}_{\left\{\eta_{b}=1\right\}}\right) \geq c \mu\left(\mathbb{1}_{\left\{\overline{\boldsymbol{\eta}}_{\mathbb{B}}\right\}} \mathbb{1}_{\left\{\eta_{b}=0\right\}}\right) .
$$

If $\mu \in \widetilde{\mathcal{G}}^{\mathrm{GRC}}$, the inequality (6.5) is implied by

$$
\mu_{\mathbb{D}}^{\mathrm{GRC}}\left(\mathbb{1}_{\left\{\overline{\boldsymbol{\eta}}_{\mathbb{B}}\right\}} \mathbb{1}_{\left\{\eta_{b}=1\right\}} \mid \boldsymbol{\eta}_{\mathbb{D}^{\mathrm{c}}}\right) \geq c \mu_{\mathbb{D}}^{\mathrm{GRC}}\left(\mathbb{1}_{\left\{\overline{\boldsymbol{\eta}}_{\mathbb{B}}\right\}} \mathbb{1}_{\left\{\eta_{b}=0\right\}} \mid \boldsymbol{\eta}_{\mathbb{D}^{\mathrm{c}}}\right) .
$$

for at least one $\mathbb{D} \supset \mathbb{B} \cup\{b\}$. Indeed, it suffices to integrate (6.6) by $\mu$ using the DLR equation (2.12).

If, on the other hand, $\mu$ is obtained as a weak limit of finite volume GRC measures, $\mu=$ $\lim _{n \rightarrow \infty} \mu_{\mathbb{B}_{n}}^{\mathrm{GRC}}\left(\cdot \mid \boldsymbol{\eta}_{n}\right)$, then the inequality (6.5) follows from (6.6) as well, provided (6.6) holds for all sufficiently large $\mathbb{D}=\mathbb{B}_{n} \supset \mathbb{B} \cup\{b\}$ and boundary conditions $\boldsymbol{\eta}_{\mathbb{B}_{n}^{c}}=\boldsymbol{\eta}_{n}$. Indeed, for all $\epsilon>0$ and all sufficiently large $n$ we have

$$
\left|\mu_{\mathbb{B}_{n}}^{\mathrm{GRC}}\left(\mathbb{1}_{\left\{\overline{\boldsymbol{\eta}}_{\mathbb{B}}\right\}} \mathbb{1}_{\left\{\eta_{b}=1\right\}} \mid \boldsymbol{\eta}_{n}\right)-\mu\left(\mathbb{1}_{\left\{\overline{\boldsymbol{\eta}}_{\mathbb{B}}\right\}} \mathbb{1}_{\left\{\eta_{b}=1\right\}}\right)\right| \leq \epsilon,
$$

and

$$
\left|\mu_{\mathbb{B}_{n}}^{\mathrm{GRC}}\left(\mathbb{1}_{\left\{\overline{\boldsymbol{\eta}}_{\mathbb{B}}\right\}} \mathbb{1}_{\left\{\eta_{b}=0\right\}} \mid \boldsymbol{\eta}_{n}\right)-\mu\left(\mathbb{1}_{\left\{\overline{\boldsymbol{\eta}}_{\mathbb{B}}\right\}} \mathbb{1}_{\left\{\eta_{b}=0\right\}}\right)\right| \leq \epsilon .
$$

Combined with (6.6), we get

$$
\mu\left(\mathbb{1}_{\left\{\overline{\boldsymbol{\eta}}_{\mathbb{B}}\right\}} \mathbb{1}_{\left\{\eta_{b}=1\right\}}\right) \geq c \mu\left(\mathbb{1}_{\left\{\overline{\boldsymbol{\eta}}_{\mathbb{B}}\right\}} \mathbb{1}_{\left\{\eta_{b}=0\right\}}\right)-\epsilon(1+c) .
$$

Since $\epsilon$ can be made arbitrary small by choosing $n$ large enough, we again obtain (6.5).

To get (6.6), we evaluate the infimum of the ratio

$$
\frac{\mu_{\mathbb{D}}^{\mathrm{GRC}}\left(\eta_{b}=1, \boldsymbol{\eta}_{\mathbb{D} \backslash\{b\}} \mid \boldsymbol{\eta}_{\mathbb{D}^{\mathrm{c}}}\right)}{\mu_{\mathbb{D}}^{\mathrm{GRC}}\left(\eta_{b}=0, \boldsymbol{\eta}_{\mathbb{D} \backslash\{b\}} \mid \boldsymbol{\eta}_{\mathbb{D}^{\mathrm{c}}}\right)}
$$

over all $\boldsymbol{\eta}_{\mathbb{D} \backslash\{b\}}$. Let us consider, for any $\boldsymbol{\eta}$ with $\eta_{b}=0$, the components $C_{x}(\boldsymbol{\eta})$ and $C_{y}(\boldsymbol{\eta})$ attached to the endpoints $x$ and $y$ of the bond $b=\langle x, y\rangle$. If $C_{x}(\boldsymbol{\eta})=C_{y}(\boldsymbol{\eta})$, using (3.6) we immediately see that the ratio (6.10) equals $e^{\beta J}-1$. On the other hand, if $C_{x}(\boldsymbol{\eta})$ and $C_{y}(\boldsymbol{\eta})$ are different components of the graph $\left(\mathbb{Z}^{d}, \mathbb{B}_{\text {occ }}(\boldsymbol{\eta})\right)$, then

$$
\frac{\mu_{\mathbb{D}}^{\mathrm{GRC}}\left(\eta_{b}=1, \boldsymbol{\eta}_{\mathbb{D} \backslash\{b\}} \mid \boldsymbol{\eta}_{\mathbb{D}^{c}}\right)}{\mu_{\mathbb{D}}^{\mathrm{GRC}}\left(\eta_{b}=0, \boldsymbol{\eta}_{\mathbb{D} \backslash\{b\}} \mid \boldsymbol{\eta}_{\mathbb{D}^{c}}\right)} \geq\left(e^{\beta J}-1\right) \frac{\sum_{m \in Q_{\max }(h)} q_{m}}{\left(\sum_{m=1}^{q} q_{m}\right)^{2}}
$$

since

$$
\frac{\sum_{m=1}^{q} q_{m} e^{-\beta\left(h_{m}-h_{\max }\right)\left(\left|\mathbb{V}\left(C_{x}(\boldsymbol{\eta})\right)\right|+\left|\mathbb{V}\left(C_{y}(\boldsymbol{\eta})\right)\right|\right)}}{\left(\sum_{m=1}^{q} q_{m} e^{-\beta\left(h_{m}-h_{\max }\right)\left|\mathbb{V}\left(C_{x}(\boldsymbol{\eta})\right)\right|}\right)\left(\sum_{m=1}^{q} q_{m} e^{-\beta\left(h_{m}-h_{\max }\right)\left|\mathbb{V}\left(C_{y}(\boldsymbol{\eta})\right)\right|}\right)} \geq \frac{\sum_{m \in Q_{\max }(h)} q_{m}}{\left(\sum_{m=1}^{q} q_{m}\right)^{2}}
$$


by the obvious fact that $0 \leq e^{-\beta\left(h_{m}-h_{\max }\right)} \leq 1$.

Proof of Theorem 3.3. Since the positive finite energy condition has been established in both relevant cases, the result follows immediately from Theorem 1 in [13].

In order to prove the corollary to Theorem 3.3, we will prove a lemma that states that the specifications $\mu_{\mathbb{B}}^{\mathrm{GRC}}$ are "almost surely quasilocal" in the language of $[16,21]$. For finite sets $\Lambda, \Delta$ with $\Lambda \subset \Delta \subset \mathbb{Z}^{d}$, let $\mathcal{M}_{\Delta, \Lambda}$ be the event

$$
\mathcal{M}_{\Delta, \Lambda}=\left\{\boldsymbol{\eta}: \forall x, y \in \Lambda \quad x \leftrightarrow \Delta^{\mathrm{c}} \text { and } y \leftrightarrow \Delta^{\mathrm{c}} \text { implies } x \underset{\mathbb{B}_{0}(\Delta)}{\overleftrightarrow{(} y\}}\right.
$$

where $x \underset{\mathbb{B}_{0}(\Delta)}{\overleftrightarrow{\longrightarrow}} y$ is the event that there is a path of occupied bonds in $\mathbb{B}_{0}(\Delta)$ connecting $x$ and $y$.

Lemma 6.2 (i) Let $\mathbb{B} \subset \mathbb{B}_{0}\left(\mathcal{Z}^{d}\right)$ be a finite set, and let $f$ be a cylinder function depending only on the bonds in $\mathbb{B}$. Then the function

$$
\boldsymbol{\eta} \mapsto \mathbb{1}_{\mathcal{M}_{\Delta, \Lambda}}(\boldsymbol{\eta}) \mu_{\mathbb{B}}^{\mathrm{GRC}}\left(f \mid \boldsymbol{\eta}_{\mathbb{B}^{c}}\right)
$$

is quasilocal for any pair of finite sets $\Delta, \Lambda$ with $\Delta \supset \Lambda \supset \mathbb{V}(\mathbb{B})$.

(ii) Let $\mu$ is a GRC limit state or a GRC Gibbs state with at most one infinite cluster and $\Lambda \subset \mathbb{Z}^{d}$ is finite, then

$$
\mu\left(\mathcal{M}_{\Delta, \Lambda}\right) \uparrow 1 \quad \text { as } \quad \Delta \uparrow \mathbb{Z}^{d}
$$

Proof. Recalling the definition of $\mu_{\mathbb{B}}^{\mathrm{GRC}}\left(\cdot \mid \boldsymbol{\eta}_{\mathbb{B}^{c}}\right)$ in terms of (3.6), we note that it is enough to prove that the function $\boldsymbol{\eta} \mapsto \mathbb{1}_{\mathcal{M}_{\Delta, \Lambda}}(\boldsymbol{\eta}) W_{\mathbb{B}}^{\mathrm{GRC}}\left(\overline{\boldsymbol{\eta}}_{\mathbb{B}} \mid \boldsymbol{\eta}_{\mathbb{B}^{c}}\right)$ is quasilocal for all $\overline{\boldsymbol{\eta}}_{\mathbb{B}} \in\{0,1\}^{\mathbb{B}}$. Let $\widetilde{\Delta} \supset \Delta$, and let $\boldsymbol{\eta}$ and $\boldsymbol{\eta}^{b}$ be two configurations differing at a single bond $b \in \mathbb{B}(\widetilde{\Delta})^{\mathrm{c}}, \eta_{b}=$ $0, \eta_{b}^{b}=1$. Suppose that $\boldsymbol{\eta} \in \mathcal{M}_{\Delta, \Lambda}$ is such that and that there is a cluster $C$ connecting $\Lambda$ with $\mathbb{B}(\widetilde{\Delta})^{\mathrm{c}}$. By the definition (6.13) of $\mathcal{M}_{\Delta, \Lambda}$, the configuration $\boldsymbol{\eta}^{b}$ also satisfies these conditions, and the component $C$ of $\left(\mathbb{Z}^{d}, \mathbb{B}_{\text {occ }}(\boldsymbol{\eta})\right)$ connecting $\Lambda$ with $\mathbb{B}(\widetilde{\Delta})^{\mathrm{c}}$ is unique. Moreover, the value of $W_{\mathbb{B}}^{\mathrm{GRC}}\left(\overline{\boldsymbol{\eta}}_{\mathbb{B}} \mid \boldsymbol{\eta}_{\mathbb{B}^{\mathrm{B}}}\right)$ is clearly not affected by changing from $\boldsymbol{\eta}$ to $\boldsymbol{\eta}^{b}$ unless $\mathbb{V}(\{b\}) \cap \mathbb{V}(C) \neq \emptyset$. Suppose that the latter occurs and denote by $C^{b}$ the corresponding component under $\boldsymbol{\eta}^{b}$. Then

$$
\begin{aligned}
\left|W_{\mathbb{B}}^{\mathrm{GRC}}\left(\overline{\boldsymbol{\eta}}_{\mathbb{B}} \mid \boldsymbol{\eta}_{\mathbb{B}^{\mathrm{c}}}^{b}\right)-W_{\mathbb{B}}^{\mathrm{GRC}}\left(\overline{\boldsymbol{\eta}}_{\mathbb{B}} \mid \boldsymbol{\eta}_{\mathbb{B}^{\mathrm{c}}}\right)\right| & \leq\left(e^{\beta J}-1\right)^{\left|\mathbb{B}_{\mathrm{occ}}\left(\overline{\boldsymbol{\eta}}_{\mathbb{B}}\right) \cap \mathbb{B}\right|} \\
& \times \sum_{m=1}^{q} q_{m}\left|e^{\beta\left(h_{m}-h_{\max }\right)\left|\mathbb{V}\left(C^{b}\right)\right|}-e^{\beta\left(h_{m}-h_{\max }\right)|\mathbb{V}(C)|}\right| .
\end{aligned}
$$

It turns out that the r.h.s. of (6.16) is exponentially small in $\operatorname{dist}(b, \Lambda)$. Indeed, for the terms with $h_{m}<h_{\max }$, both terms between the absolute value signs go to zero exponentially fast, while for $h_{m}=h_{\max }$ both terms tend exponentially fast to one as $\operatorname{dist}(b, \Lambda) \rightarrow \infty$. Thus, the r.h.s. of (6.16) is summable over the positions of $b$. By the standard telescoping trick, this proves quasilocality (i.e., continuity in the product topology) of the function $\boldsymbol{\eta} \mapsto$ $\mathbb{1}_{\mathcal{M}_{\Delta, \Lambda}}(\boldsymbol{\eta}) W_{\mathbb{B}}^{\mathrm{GRC}}\left(\overline{\boldsymbol{\eta}}_{\mathbb{B}} \mid \boldsymbol{\eta}_{\mathbb{B}^{c}}\right)$, as required by (i).

(ii) Since $\mathcal{M}_{\Delta, \Lambda} \uparrow \mathcal{M}_{\Lambda}$, where $\mathcal{M}_{\Lambda}$ is the set of configurations featuring at most one infinite component incident with $\Lambda$, we have that $\mu\left(\mathcal{M}_{\Delta, \Lambda}\right) \uparrow \mu\left(\mathcal{M}_{\Lambda}\right)=1$, by the assumption that $\mu$ has at most one infinite cluster. 
Proof of Corollary to Theorem 3.3. Let $\mu=\lim _{n \rightarrow \infty} \mu_{\mathbb{B}_{n}}^{\mathrm{GRC}}\left(\cdot \mid \boldsymbol{\eta}_{n}\right)$ be a translation invariant GRC limit state. It is not hard to verify that $\mu_{n}=\mu_{\mathbb{B}_{n}}^{\mathrm{GRC}}\left(\cdot \mid \boldsymbol{\eta}_{n}\right)$ satisfies the DLR condition

$$
\mu_{n}(f)=\int \mu_{n}(\mathrm{~d} \boldsymbol{\eta}) \mu_{\mathbb{B}}^{\mathrm{GRC}}\left(f \mid \boldsymbol{\eta}_{\mathbb{B}^{\mathrm{c}}}\right),
$$

for any $\mathbb{B}$-cylinder function $f$ and any $\mathbb{B} \subset \mathbb{B}_{n}$. Since the specifications $\mu_{\mathbb{B}}^{\mathrm{GRC}}(f \mid \cdot)$ are not quasilocal, this does not imply, however, that the limiting measure $\mu$ satisfies the DLR equation. To circumvent this problem, we follow the strategy of [21] and [16] involving the "almost sure quasilocality" of $\mu_{\mathbb{B}}^{\mathrm{GRC}}(f \mid \cdot)$.

Let $\mathbb{B}$ be a finite set of bonds, and let $f$ be a bounded $\mathbb{B}$-cylinder function. Since both $f$ and $\mathbb{1}_{\mathcal{M}_{\Delta, \mathrm{V}(\mathbb{B})}}(\cdot) \mu_{\mathbb{B}}^{\mathrm{GRC}}(f \mid \cdot)$ are quasilocal for all $\Delta \supset \mathbb{V}(\mathbb{B})$, we have

$$
\mu\left(\mathbb{1}_{\mathcal{M}_{\Delta, \mathbb{V}(\mathbb{B})}}(\cdot) \mu_{\mathbb{B}}^{\mathrm{GRC}}(f \mid \cdot)\right)=\lim _{n \rightarrow \infty} \mu_{n}\left(\mathbb{1}_{\mathcal{M}_{\Delta, \mathbb{V}(\mathbb{B})}}(\cdot) \mu_{\mathbb{B}}^{\mathrm{GRC}}(f \mid \cdot)\right)
$$

and

$$
\mu(f)=\lim _{n \rightarrow \infty} \mu_{n}(f)=\lim _{n \rightarrow \infty} \mu_{n}\left(\mu_{\mathbb{B}}^{\mathrm{GRC}}(f \mid \cdot)\right),
$$

where we have used (6.17) in the last step.

Let $\epsilon>0$. By Theorem 3.3, $\mu$ has a unique infinite cluster, which allows us to use (6.15). Combined with the boundedness of $\mu_{\mathbb{B}}^{\mathrm{GRC}}(f \mid \cdot)$, we can therefore choose $\Delta_{1}, \Delta_{2}$ and $n_{0}$ such that

$$
\left|\mu\left(\mu_{\mathbb{B}}^{\mathrm{GRC}}(f \mid \cdot)\right)-\mu\left(\mathbb{1}_{\mathcal{M}_{\Delta, \mathbb{V}(\mathbb{B})}}(\cdot) \mu_{\mathbb{B}}^{\mathrm{GRC}}(f \mid \cdot)\right)\right| \leq \frac{\epsilon}{2}
$$

and

$$
\left|\mu_{n}\left(\mu_{\mathbb{B}}^{\mathrm{GRC}}(f \mid \cdot)\right)-\mu_{n}\left(\mathbb{1}_{\mathcal{M}_{\Delta, \mathrm{V}(\mathbb{B})}}(\cdot) \mu_{\mathbb{B}}^{\mathrm{GRC}}(f \mid \cdot)\right)\right| \leq \frac{\epsilon}{2}
$$

provided $\Delta_{1} \subset \Delta \subset \Delta_{2}$ and $n \geq n_{0}$. Combining (6.18) - (6.21), we get

$$
\left|\mu(f)-\mu\left(\mu_{\mathbb{B}}^{\mathrm{GRC}}(f \mid \cdot)\right)\right| \leq \epsilon .
$$

Since $\epsilon$ was arbitrary, we get that $\mu(f)=\mu\left(\mu_{\mathbb{B}}^{\mathrm{GRC}}(f \mid \cdot)\right)$, i.e., $\mu \in \mathcal{G}^{\mathrm{GRC}}$.

Proof of Theorem 3.4. To prove Theorem 3.4, we will prove that for all finite sets of bonds $\mathbb{B}_{1}$ and $\mathbb{B}_{2}$ with $\mathbb{B}_{1} \cap \mathbb{B}_{2}=\emptyset$, and for all bounded cylinder functions $f$ and $g$ depending only on the bonds in $\mathbb{B}_{1}$ and $\mathbb{B}_{2}$, respectively, we have

$$
\mu(g f)=\mu\left(g \mu_{\mathbb{B}_{1}}^{\mathrm{GRC}}(f \mid \cdot)\right),
$$

provided $\mu$ has at most one infinite cluster with probability one.

In a first step, we use the DLR equation (2.11) and the consistency of the specifications $\left\{\mu_{\mathbb{B}}^{\mathrm{GRC}}\right\}$ to conclude that for $\mathbb{B} \supset \mathbb{B}_{1} \cup \mathbb{B}_{2}$ we have

$$
\begin{aligned}
\mu(g f) & =\int \mu(\mathrm{d} \boldsymbol{\eta}) \mu_{\mathbb{B}}^{\mathrm{GRC}}\left(g f \mid \boldsymbol{\eta}_{\mathbb{B}^{\mathrm{c}}}\right) \\
& =\int \mu(\mathrm{d} \boldsymbol{\eta}) \mu_{\mathbb{B}}^{\mathrm{GRC}}\left(g \mu_{\mathbb{B}_{1}}^{\mathrm{GRC}}(f \mid \cdot) \mid \boldsymbol{\eta}_{\mathbb{B}^{\mathrm{c}}}\right) .
\end{aligned}
$$

Next let $\Delta \supset \mathbb{V}\left(\mathbb{B}_{1}\right)$, and let $\mathcal{M}_{\Delta, \mathbb{V}\left(\mathbb{B}_{1}\right)}$ be the event introduced in (6.13). Since both $g$ and $\mathbb{1}_{\mathcal{M}_{\Delta, \mathbb{V}\left(\mathbb{B}_{1}\right)}}(\cdot) \mu_{\mathbb{B}_{1}}^{\mathrm{GRC}}(f \mid \cdot)$ are quasilocal, we have

$$
\lim _{\mathbb{B} \nearrow \mathbb{B}_{0}\left(\mathbb{Z}^{d}\right)} \int \mu(\mathrm{d} \boldsymbol{\eta}) \mu_{\mathbb{B}}^{\mathrm{GRC}}\left(g \mathbb{1}_{\mathcal{M}_{\Delta, \mathbb{v}\left(\mathbb{B}_{1}\right)}} \mu_{\mathbb{B}_{1}}^{\mathrm{GRC}}(f \mid \cdot) \mid \boldsymbol{\eta}_{\mathbb{B}^{c}}\right)=\mu\left(g \mathbb{1}_{\mathcal{M}_{\Delta, \mathbb{v}\left(\mathbb{B}_{1}\right)}} \mu_{\mathbb{B}_{1}}^{\mathrm{GRC}}(f \mid \cdot)\right) .
$$


Here, we have used the fact that as a quasilocal function, the function $g \mathbb{1}_{\mathcal{M}_{\Delta, \mathrm{V}\left(\mathbb{B}_{1}\right)}} \mu_{\mathbb{B}_{1}}^{\mathrm{GRC}}(f \mid \cdot)$ can be approximated arbitrarily well by local functions, and then we have applied the DLR equation (2.11) for local functions. To complete the proof, we use that $\mu\left(\mathcal{M}_{\Delta, \mathbb{V}\left(\mathbb{B}_{1}\right)}\right) \uparrow \mu\left(N_{\infty} \leq 1\right)=1$ as $\Delta \uparrow \mathbb{Z}^{d}$ by Lemma 6.2. Since $f$ and $g$ are bounded, we conclude that for all $\epsilon>0$ we can choose $\Delta$ in such a way that

$$
\left|\int \mu(\mathrm{d} \boldsymbol{\eta}) \mu_{\mathbb{B}}^{\mathrm{GRC}}\left(g \mu_{\mathbb{B}_{1}}^{\mathrm{GRC}}(f \mid \cdot) \mid \boldsymbol{\eta}_{\mathbb{B}^{c}}\right)-\int \mu(\mathrm{d} \boldsymbol{\eta}) \mu_{\mathbb{B}}^{\mathrm{GRC}}\left(g \mathbb{1}_{\mathcal{M}_{\Delta, \mathbb{V}\left(\mathbb{B}_{1}\right)}} \mu_{\mathbb{B}_{1}}^{\mathrm{GRC}}(f \mid \cdot) \mid \boldsymbol{\eta}_{\mathbb{B}^{c}}\right)\right| \leq \frac{\epsilon}{2}
$$

and

$$
\left|\mu\left(g \mathbb{1}_{\mathcal{M}_{\Delta, \mathrm{V}\left(\mathbb{B}_{1}\right)}} \mu_{\mathbb{B}_{1}}^{\mathrm{GRC}}(f \mid \cdot)\right)-\mu\left(g \mu_{\mathbb{B}_{1}}^{\mathrm{GRC}}(f \mid \cdot)\right)\right| \leq \frac{\epsilon}{2},
$$

provided $\mathbb{B} \supset \mathbb{B}(\Delta)$. Combined with (6.24) and (6.25) this proves that

$$
\left|\mu(g f)-\mu\left(g \mu_{\mathbb{B}_{1}}^{\mathrm{GRC}}(f \mid \cdot)\right)\right| \leq \epsilon .
$$

Since $\epsilon$ was arbitrary, this completes the proof of (6.23) and hence the proof of Theorem 3.4.

Proof of Theorem 2.3. As pointed out in the remark after Theorem 2.3, the statements of the theorem are special cases of those in Theorem 3.1(ii), Theorem 3.3 and its corollary.

\section{Weak Limits of the ES GibBs Measures}

Since by Theorem 2.3(i) the limits (2.17) and (2.18) exist for every quasilocal $f$ depending only on the bond configurations $\boldsymbol{\eta}$, to prove Theorem 2.4 we just need to extend this to functions of both $\boldsymbol{\sigma}$ and $\boldsymbol{\eta}$. In this regard, it will turn out to be useful to swap the $\boldsymbol{\sigma}$-dependence and $\boldsymbol{\eta}$-dependence under the expectation w.r.t. the ES Gibbs measures. Before we formulate this precisely, let us give some definitions.

For any collection $\left\{\mathcal{F}_{i}\right\}_{i=1}^{q}$ of pairwise disjoint finite sets $\mathcal{F}_{i} \subset \mathbb{Z}^{d}$, let us define

$$
F_{\left\{\mathcal{F}_{i}\right\}}^{\text {free }}(\boldsymbol{\eta})=\prod_{i<j} \mathbb{1}_{\left\{\mathcal{F}_{i} \leftrightarrow \mathcal{F}_{j}\right\}}(\boldsymbol{\eta}) \prod_{m=1}^{q} \prod_{\substack{C: \dot{\mathcal{F}}_{m} \neq \emptyset \\ \mathbb{V}(C)}} \frac{e^{\beta h_{m}|\mathbb{V}(C)|}}{\Theta_{\text {free }}(C)} .
$$

Here, $\mathbb{1}_{\left\{\mathcal{F}_{i} \leftrightarrow \mathcal{F}_{j}\right\}}(\boldsymbol{\eta})$ is the indicator of the event that, under $\boldsymbol{\eta}$, no point in $\mathcal{F}_{i}$ is connected to any point in $\mathcal{F}_{j}$ by a path of occupied bonds, the product over $C$ runs over all components of the set $\mathbb{B}_{\text {occ }}(\boldsymbol{\eta})$ with $\mathbb{V}(C) \cap \mathcal{F}_{m} \neq \emptyset$, and $\Theta_{\text {free }}(C)$ is as in (3.2) (with $q_{m}=1$ ).

Similarly, given a finite set $\Lambda$ with $\mathcal{F}=\cup_{i=1}^{q} \mathcal{F}_{i} \subset \Lambda$, let us define

$$
F_{\Lambda,\left\{\mathcal{F}_{i}\right\}}^{\bar{m}}(\boldsymbol{\eta})=\prod_{i<j} \mathbb{1}_{\left\{\mathcal{F}_{i} \leftrightarrow \mathcal{F}_{j}\right\}}(\boldsymbol{\eta}) \prod_{m=1}^{q} \prod_{\substack{C:: \\ \mathbb{V}(C) \cap \mathcal{F}_{m} \neq \emptyset}} \frac{e^{\beta h_{m}|\mathbb{V}(C)|}}{\Theta_{\Lambda, m}(C)} \chi_{\Lambda, \bar{m}}(C, m)
$$

for each $\bar{m} \in\{1, \ldots, q\}$, where we recall the definitions (3.5) and use $\chi_{\Lambda, \bar{m}}(C, m)$ to denote

$$
\chi_{\Lambda, \bar{m}}(C, m)= \begin{cases}1 & \mathbb{V}(C) \cap \Lambda^{\mathrm{c}}=\emptyset \text { or } m=\bar{m} \\ 0 & \text { otherwise. }\end{cases}
$$

Remark. In the following, it will be important to remember explicitly from which value of the boundary spin the measure $\mu_{\Lambda \text {,maxwir }}^{\mathrm{RC}}$ originated. Therefore we shall temporarily write $\mu_{\Lambda, m}^{\mathrm{RC}}$ instead of $\mu_{\Lambda, \text { maxwir }}^{\mathrm{RC}}$. 
Lemma 7.1 Let $A \subset \mathbb{Z}^{d}$ be a finite set and let $f$ be a cylinder function in $(A, \mathbb{B}(A))$. Then there are numbers $\left(a_{\left\{\mathcal{F}_{i}\right\}}\right)$ such that

$$
\begin{aligned}
\mu_{\Lambda, \text { free }}^{\mathrm{ES}}(f) & =\sum_{\left\{\mathcal{F}_{i}\right\}} a_{\left\{\mathcal{F}_{i}\right\}} \mu_{\Lambda, \text { free }}^{\mathrm{RC}}\left(F_{\left\{\mathcal{F}_{i}\right\}}^{\text {free }}\right) \\
\mu_{\Lambda, \bar{m}}^{\mathrm{ES}}(f) & =\sum_{\left\{\mathcal{F}_{i}\right\}} a_{\left\{\mathcal{F}_{i}\right\}} \mu_{\Lambda, \bar{m}}^{\mathrm{RC}}\left(F_{\Lambda,\left\{\mathcal{F}_{i}\right\}}^{\bar{m}}\right)
\end{aligned}
$$

for each $\bar{m} \in\{1, \ldots, q\}$ and all $\Lambda \supset A$ with $\mathbb{B}_{0}(\Lambda) \supset \mathbb{B}(A)$. Moreover, $a_{\left\{\mathcal{F}_{i}\right\}}=0$ whenever there is an $x \in \mathcal{F}=\cup_{i=1}^{q} \mathcal{F}_{i}$ with $\operatorname{dist}(x, A)>1$. In particular, both sums above are finite.

Proof. Let $\Lambda$ be such that $\Lambda \supset A$ and $\mathbb{B}_{0}(\Lambda) \supset \mathbb{B}(A)$. Then by using that $\mu_{\Lambda, \text { free }}^{\mathrm{ES}}$ and $\mu_{\Lambda, \bar{m}}^{\mathrm{ES}}$ are Gibbs measures we have

$$
\mu_{\Lambda, \text { free }}^{\mathrm{ES}}(f)=\mu_{\Lambda, \text { free }}^{\mathrm{ES}}\left(\mu_{A, \mathbb{B}(A)}^{\mathrm{ES}}\left(f \mid \boldsymbol{\sigma}_{A^{\mathrm{c}}}, \boldsymbol{\eta}_{\mathbb{B}(A)^{\mathrm{c}}}\right)\right),
$$

and similarly for $\mu_{\Lambda, \bar{m}}^{\mathrm{ES}}(f)$. The finite volume specification $\mu_{A, \mathbb{B}(A)}^{\mathrm{ES}}\left(f \mid \boldsymbol{\sigma}_{A^{\mathrm{c}}}, \boldsymbol{\eta}_{\mathbb{B}(A)^{\mathrm{c}}}\right)$ depends only

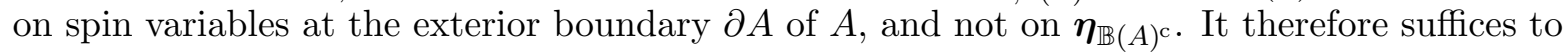
prove the claim for functions of the spin variables that are supported in $\bar{A}=A \cup \partial A$.

Each such function $f$ can be uniquely recast as $\sum_{\left\{\mathcal{F}_{i}\right\}} a_{\left\{\mathcal{F}_{i}\right\}} f_{\left\{\mathcal{F}_{i}\right\}}$, where $a_{\left\{\mathcal{F}_{i}\right\}}$ are real numbers such that $a_{\left\{\mathcal{F}_{i}\right\}}=0$ whenever $\mathcal{F} \not \subset \bar{A}$, and

$$
f_{\left\{\mathcal{F}_{i}\right\}}(\boldsymbol{\sigma})=\prod_{m=1}^{q} \prod_{x \in \mathcal{F}_{m}} \delta_{\sigma_{x}, m} .
$$

It is now a matter of a direct computation to show that, for all $\bar{m} \in\{1, \ldots, q\}$,

$$
\begin{aligned}
\mu_{\Lambda, \text { free }}^{\mathrm{ES}}\left(f_{\left\{\mathcal{F}_{i}\right\}} \mid \boldsymbol{\eta}\right) & =F_{\left\{\mathcal{F}_{i}\right\}}^{\mathrm{free}}(\boldsymbol{\eta}) \\
\mu_{\Lambda, \bar{m}}^{\mathrm{ES}}\left(f_{\left\{\mathcal{F}_{i}\right\}} \mid \boldsymbol{\eta}\right) & =F_{\Lambda,\left\{\mathcal{F}_{i}\right\}}^{\bar{m}}(\boldsymbol{\eta}) .
\end{aligned}
$$

Namely, the components $C$ of $\mathbb{B}_{\mathrm{occ}}(\boldsymbol{\eta})$ such that $\mathbb{V}(C) \cap \mathcal{F}_{m} \neq \emptyset$ necessarily satisfy that $\mathbb{V}(C) \cap \mathcal{F}_{i}=\emptyset$ for all $i \neq m$. This gives rise to the indicators $\mathbb{1}_{\left\{\mathcal{F}_{i} \leftrightarrow \mathcal{F}_{j}\right\}}$. For $\boldsymbol{\eta}$ such that $\prod_{i<j} \mathbb{1}_{\left\{\mathcal{F}_{i} \leftrightarrow \mathcal{F}_{j}\right\}}(\boldsymbol{\eta})=1$, the spin configuration can be integrated out, yielding the ratios $e^{\beta h_{m}|\mathbb{V}(C)|} / \Theta_{\text {free }}(C)$ resp. $e^{\beta h_{m}|\mathbb{V}(C)|} / \Theta_{\Lambda, \bar{m}}(C)$. However, one gets the latter only when $\mathbb{V}(C) \cap \Lambda^{\mathrm{c}}=\emptyset$ or $m=\bar{m}$. The claim is finished by taking the expectation w.r.t. $\boldsymbol{\eta}$.

It was shown in Lemma 7.1 that $\boldsymbol{\sigma}$-dependent cylinder functions can be interchanged under the expectation for $\boldsymbol{\eta}$-dependent functions $F_{\left\{\mathcal{F}_{i}\right\}}^{\text {free }}$ and $F_{\Lambda,\left\{\mathcal{F}_{i}\right\}}^{m}$. Unfortunately, the weak limits (2.17) and (2.18) cannot yet be invoked to conclude the existence of (2.19) and (2.20), the reason being that the $F_{\left\{\mathcal{F}_{i}\right\}}$ 's are, in general, not quasilocal. (Moreover, $F_{\Lambda,\left\{\mathcal{F}_{i}\right\}}^{m}$ even depends explicitly on the expanding volume.) However, both functions $F_{\left\{\mathcal{F}_{i}\right\}}^{\text {free }}$ and $F_{\Lambda,\left\{\mathcal{F}_{i}\right\}}^{m}$ turn out to be "almost surely" quasilocal, in the terminology of [21] and [16], which is still sufficient for the limits (2.17) and (2.18) to exist.

For finite sets $\mathcal{F}, \Delta$ with $\mathcal{F} \subset \Delta$, let $\mathcal{M}_{\Delta, \mathcal{F}}$ be the event define in (6.13). Let further

$$
\mathcal{M}_{\Delta,\left\{\mathcal{F}_{i}\right\}}^{m}=\left\{\boldsymbol{\eta} \in \mathcal{M}_{\Delta, \mathcal{F}}: x \in \mathcal{F} \text { with } x \leftrightarrow \Delta^{\mathrm{c}} \text { implies } x \in \mathcal{F}_{m}\right\},
$$


and recall $q_{0}=\#\left\{m: h_{m}=h_{\max }\right\}$. For each $\Delta, m \in\{1, \ldots, q\}$, and $\left\{\mathcal{F}_{i}\right\}$ define also a random variable $Q_{\Delta,\left\{\mathcal{F}_{i}\right\}}^{m}$ by putting

$$
Q_{\Delta,\left\{\mathcal{F}_{i}\right\}}^{m}= \begin{cases}q_{0} & \mathcal{F}_{m} \leftrightarrow \Delta^{\mathrm{c}} \\ 1 & \text { otherwise. }\end{cases}
$$

The remainder of the proof is based on an approximation of $F_{\left\{\mathcal{F}_{i}\right\}}$ 's by quasilocal functions and showing that the error incurred thereby upon the expectations of $F_{\left\{\mathcal{F}_{i}\right\}}$ 's is negligible. These claims are formulated in Lemma 7.2 and Lemma 7.3 below.

Lemma 7.2 For all finite $\Delta \subset \mathbb{Z}^{d}$ and any $\left\{\mathcal{F}_{i}\right\}$ with $\mathcal{F}=\cup_{i} \mathcal{F}_{i}$

(i) $F_{\left\{\mathcal{F}_{i}\right\}}^{\text {free }} \mathbb{1}_{\mathcal{M}_{\Delta,\left\{\mathcal{F}_{i}\right\}}^{m}}$ is quasilocal for all $m \in\{1, \ldots, q\}$.

(ii) $F_{\left\{\mathcal{F}_{i}\right\}}^{\text {free }} \mathbb{1}_{\mathcal{M}_{\Delta, \mathcal{F}}}$ is quasilocal.

Proof. (i) Let $m$ be fixed and let $\Lambda \supset \Delta$. Observe that $\mathbb{1}_{\mathcal{M}_{\Delta,\left\{\mathcal{F}_{i}\right\}}^{m}} \prod_{i<j} \mathbb{1}_{\left\{\mathcal{F}_{i} \leftrightarrow \mathcal{F}_{j}\right\}}$ is a cylinder function in $\mathbb{B}(\Lambda)$. Hence, only the contributions from the product over the connected components in $(7.1)$ can be altered by flipping a bond $b \notin \mathbb{B}(\Lambda)$. Let us estimate precisely the incurred change.

Let $\boldsymbol{\eta}$ and $\boldsymbol{\eta}^{b}$ be two configurations differing at a single bond $b \in \mathbb{B}(\Lambda)^{\mathrm{c}}, \eta_{b}=0, \eta_{b}^{b}=1$. Suppose that $\boldsymbol{\eta} \in \mathcal{M}_{\Delta,\left\{\mathcal{F}_{i}\right\}}^{m}$ is such that $\prod_{i<j} \mathbb{1}_{\left\{\mathcal{F}_{i} \leftrightarrow \mathcal{F}_{j}\right\}}(\boldsymbol{\eta})=1$ and that there is a $C$ connecting $\mathcal{F}_{m}$ with $\mathbb{B}(\Lambda)^{\mathrm{c}}$. By the definition (7.9) of $\mathcal{M}_{\Delta,\left\{\mathcal{F}_{i}\right\}}^{m}$, the configuration $\boldsymbol{\eta}^{b}$ also satisfies these three conditions, and by the definition (6.13) of $\mathcal{M}_{\Delta,\{\mathcal{F}\}}$, the component $C$ of $\left(\mathbb{Z}^{d}, \mathbb{B}_{\text {occ }}(\boldsymbol{\eta})\right)$ connecting $\mathcal{F}_{m}$ and $\mathbb{B}(\Lambda)^{\mathrm{c}}$ is unique. Moreover, the value of $F_{\left\{\mathcal{F}_{i}\right\}}^{\text {free }}$ is not affected by changing from $\boldsymbol{\eta}$ to $\boldsymbol{\eta}^{b}$ unless $\mathbb{V}\left(\{b\} \cap \mathbb{V}(C) \neq \emptyset\right.$. Suppose that the latter occurs and denote by $C^{b}$ the corresponding component under $\boldsymbol{\eta}^{b}$. Then

$$
\left|F_{\left\{\mathcal{F}_{i}\right\}}^{\text {free }}\left(\boldsymbol{\eta}^{b}\right)-F_{\left\{\mathcal{F}_{i}\right\}}^{\text {free }}(\boldsymbol{\eta})\right| \leq\left|\frac{e^{\beta h_{m}\left|\mathbb{V}\left(C^{b}\right)\right|}}{\Theta_{\text {free }}\left(C^{b}\right)}-\frac{e^{\beta h_{m}|\mathbb{V}(C)|}}{\Theta_{\text {free }}(C)}\right|,
$$

where we have estimated all ratios by 1 , except for the one affected by flipping $b$. As in the proof of Lemma 6.2, the r.h.s. of (7.11) is exponentially small in $\operatorname{dist}(b, \mathcal{F})$. This proves (i).

To prove (ii), it clearly suffices to note that

$$
F_{\left\{\mathcal{F}_{i}\right\}}^{\text {free }}\left[\mathbb{1}_{\mathcal{M}_{\Delta, \mathcal{F}}}-\sum_{m=1}^{q} \mathbb{1}_{\mathcal{M}_{\Delta,\left\{\mathcal{F}_{i}\right\}}^{m}}\right]
$$

is a cylinder event in $\mathbb{B}(\Delta)$. Namely, the function in the brackets is zero unless there is no component incident with $\mathcal{F}$ that reaches up to $\Delta^{\mathrm{c}}$. In that case, $F_{\left\{\mathcal{F}_{i}\right\}}^{\text {free }}$ depends only on bonds from $\mathbb{B}(\Delta)$, i.e, it is effectively a local function.

The next lemma has two parts, both of which will be needed in the proof of Theorem 2.4. It turns out that the first part can be proved for the more general GRC model.

Lemma 7.3 Let $\left\{\mathcal{F}_{i}\right\}, \mathcal{F}$ and $m$ be such that $\mathcal{F}=\cup_{i=1}^{q} \mathcal{F}_{i}$ and $h_{m}=h_{\max }$.

(i) Then

$$
\begin{aligned}
& \lim _{\Delta \nearrow \mathbb{Z}^{d}} \lim _{\Lambda \nearrow \mathbb{Z}^{d}} \mu_{\Lambda, \text { free }}^{\mathrm{GRC}}\left(\mathcal{M}_{\Delta, \mathcal{F}}\right)=1 \\
& \lim _{\Delta \nearrow \mathbb{Z}^{d} \Lambda \nearrow \mathbb{Z}^{d}} \mu_{\Lambda, m}^{\mathrm{GRC}}\left(\mathcal{M}_{\Delta, \mathcal{F}}\right)=1 .
\end{aligned}
$$


(ii) In addition, let $G_{\Lambda, \Delta, m}^{\left\{\mathcal{F}_{i}\right\}}=F_{\Lambda,\left\{\mathcal{F}_{i}\right\}}^{m} \mathbb{1}_{\mathcal{M}_{\Delta, \mathcal{F}}}-Q_{\Delta,\left\{\mathcal{F}_{i}\right\}}^{m} \mathbb{1}_{\mathcal{M}_{\Delta,\left\{\mathcal{F}_{i}\right\}}^{m}} F_{\left\{\mathcal{F}_{i}\right\}}^{\text {free }}$. Then

$$
\lim _{\Delta \nearrow \mathbb{Z}^{d}} \lim _{\Lambda \nearrow \mathbb{Z}^{d}} \mu_{\Lambda, m}^{\mathrm{RC}}\left(G_{\Lambda, \Delta, m}^{\left\{\mathcal{F}_{i}\right\}}\right)=0
$$

Proof. (i) The inner limits on the l.h.s. exist because $\mathcal{M}_{\Delta, \mathcal{F}}$ is a cylinder event, and the GRC measures have a weak limit by Theorem 3.1(ii). The outer limit is then a consequence of the fact that $\mathcal{M}_{\Delta, \mathcal{F}} \uparrow \mathcal{M}_{\mathcal{F}}$, where $\mathcal{M}_{\mathcal{F}}$ is the set of configurations featuring at most one infinite component incident with $\mathcal{F}$. The limits are thus equal to $\mu_{\text {free }}^{\mathrm{GRC}}\left(\mathcal{M}_{\mathcal{F}}\right)$ and $\mu_{m}^{\mathrm{GRC}}\left(\mathcal{M}_{\mathcal{F}}\right)$, respectively. Now, since $\mu_{\text {free }}^{\mathrm{GRC}}$ and $\mu_{m}^{\mathrm{GRC}}$ are translation invariant (as already proved Theorem 3.1) and are obtained as weak limits of finite volume GRC measures, we can apply Theorem 3.3 to assert that both these measures have almost surely at most one infinite cluster. This means $\mu_{\text {free }}^{\mathrm{GRC}}\left(\mathcal{M}_{\mathcal{F}}\right)=1=\mu_{m}^{\mathrm{GRC}}\left(\mathcal{M}_{\mathcal{F}}\right)$. By putting these observations together, (7.13) and (7.14) are proven.

To prove (ii), take $\left\{\mathcal{F}_{i}\right\}$ and $\Delta \subset \Lambda$ with $\Delta \supset \mathcal{F}$. Then the following three possibilities can occur for configurations $\boldsymbol{\eta} \in\{0,1\}^{\mathbb{B}(\Lambda)}$ :

(A) $\mathcal{F} \leftrightarrow \Delta^{\mathrm{c}}$

(B) $\mathcal{F} \leftrightarrow \Delta^{\mathrm{c}}$ but $\mathcal{F} \leftrightarrow \Lambda^{\mathrm{c}}$

(C) $\mathcal{F} \leftrightarrow \Lambda^{\mathrm{c}}$.

Clearly, under $(\mathrm{A})$, the absence of components connecting $\mathcal{F}$ with the outside of $\Delta$ implies

$$
\mathbb{1}_{\mathcal{M}_{\Delta,\left\{\mathcal{F}_{i}\right\}}^{m}}=\mathbb{1}_{\mathcal{M}_{\Delta, \mathcal{F}}}, \quad Q_{\Delta,\left\{\mathcal{F}_{i}\right\}}^{m}=1, \quad \text { and } \quad F_{\Lambda,\left\{\mathcal{F}_{i}\right\}}^{m}=F_{\left\{\mathcal{F}_{i}\right\}}^{\text {free }}
$$

by the inspection of (7.1) and (7.2). Consequently, all terms in the definition of $G_{\Lambda, \Delta, m}^{\left\{\mathcal{F}_{i}\right\}}$ cancel and $G_{\Lambda, \Delta, m}^{\left\{\mathcal{F}_{i}\right\}}=0$.

If (C) occurs then both terms contributing to $G_{\Lambda, \Delta, m}^{\left\{\mathcal{F}_{i}\right\}}$ are zero unless there is a unique component connecting $\mathcal{F}$ to $\partial \Lambda$, and this component connects $\mathcal{F}_{m}$ to $\partial \Lambda$. If we have such a component $C_{m, \Lambda}$, we get

$$
\mathbb{1}_{\mathcal{M}_{\Delta,\left\{\mathcal{F}_{i}\right\}}^{m}}=\mathbb{1}_{\mathcal{M}_{\Delta, \mathcal{F}}}, \quad Q_{\Delta,\left\{\mathcal{F}_{i}\right\}}^{m}=q_{0}, \quad \text { and } \quad F_{\Lambda,\left\{\mathcal{F}_{i}\right\}}^{m}=F_{\left\{\mathcal{F}_{i}\right\}}^{\text {free }} \frac{\Theta_{\text {free }}\left(C_{m, \Lambda}\right)}{e^{\beta h_{m}\left|\mathbb{V}\left(C_{m, \Lambda}\right)\right|}}
$$

Since $\Theta_{\text {free }}\left(C_{m, \Lambda}\right) / e^{\beta h_{m}\left|\mathbb{V}\left(C_{m, \Lambda}\right)\right|}$ is equal to $q_{0}$ plus an error term that is exponentially small in the distance between $\mathcal{F}_{m}$ and $\partial \Lambda$, this implies that $G_{\Lambda, \Delta, m}^{\left\{\mathcal{F}_{i}\right\}}$ tends to zero as $\Lambda \nearrow \mathbb{Z}^{d}$.

The proof of (7.15) therefore boils down to the analysis of (B). Let $\mathcal{P}_{\Lambda, \Delta}^{\mathcal{F}}$ denote the event (B), i.e., $\mathcal{P}_{\Lambda, \Delta}^{\mathcal{F}}=\left\{\boldsymbol{\eta}: \mathcal{F} \leftrightarrow \Delta^{\mathrm{c}}\right.$ but $\left.\mathcal{F} \leftrightarrow \Lambda^{\mathrm{c}}\right\}$. Then, by the preceding reasoning, $\left|G_{\Lambda, \Delta, m}^{\left\{\mathcal{F}_{i, m}\right.}\right| \leq q_{0} \mathbb{1}_{\mathcal{P}_{\Lambda, \Delta}^{\mathcal{F}}}$ plus an error exponentially small error term that tends to zero as $\Lambda \nearrow \mathbb{Z}^{d}$. Thus, it suffices to prove that

$$
\lim _{\Delta \nearrow \mathbb{Z}^{d}} \lim _{\Lambda \nearrow \mathbb{Z}^{d}} \mu_{\Lambda, m}^{\mathrm{RC}}\left(\mathcal{P}_{\Lambda, \Delta}^{\mathcal{F}}\right)=0
$$

We will establish this by proving that the events $(\mathrm{A})$ or $(\mathrm{C})$ get the full mass under these limits. First we recall the well known characterization

$$
\mu_{m}^{\mathrm{RC}}(\mathcal{F} \leftrightarrow \infty)=\lim _{\Lambda \nearrow \mathbb{Z}^{d}} \mu_{\Lambda, m}^{\mathrm{RC}}\left(\mathcal{F} \leftrightarrow \Lambda^{\mathrm{c}}\right)
$$


This follows from the fact that for $\Lambda \subset \widetilde{\Lambda}$ we have the inequalities $\mu_{\widetilde{\Lambda}, m}^{\mathrm{RC}}\left(\mathcal{F} \leftrightarrow \widetilde{\Lambda}^{\mathrm{c}}\right) \leq \mu_{\widetilde{\Lambda}, m}^{\mathrm{RC}}(\mathcal{F} \leftrightarrow$ $\left.\Lambda^{\mathrm{c}}\right) \leq \mu_{\Lambda, m}^{\mathrm{RC}}\left(\mathcal{F} \leftrightarrow \Lambda^{\mathrm{c}}\right)$, where the first one is due to monotonicity of $\left\{\mathcal{F} \leftrightarrow \Lambda^{\mathrm{c}}\right\}$ in $\Lambda$ and the second one is due to (4.15).

Since $\left\{\mathcal{F} \leftrightarrow \Delta^{\mathrm{c}}\right\} \uparrow\{\mathcal{F} \leftrightarrow \infty\}$ as $\Delta \nearrow \mathbb{Z}^{d}$, we easily get that

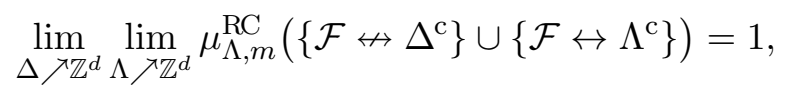

proving the desired claim.

With Lemma 7.2 and 7.3 in the hand, the proof of Theorem 2.4 can be concluded.

Proof of Theorem 2.4. By Lemma 7.1, the existence of the limits (2.19) and (2.20) has been reduced the existence of the $\operatorname{limits}_{\lim _{\Lambda} \mathbb{Z}^{d}} \mu_{\Lambda, \text { free }}^{\mathrm{RC}}\left(F_{\left\{\mathcal{F}_{i}\right\}}^{\text {free }}\right)$ and $\lim _{\Lambda \nearrow \mathbb{Z}^{d}} \mu_{\Lambda, m}^{\mathrm{RC}}\left(F_{\Lambda,\left\{\mathcal{F}_{i}\right\}}^{m}\right)$. To prove the existence of the latter, let $\epsilon>0$. Then there are finite sets $\bar{\Lambda}, \bar{\Delta}_{1}, \bar{\Delta}_{2} \subset \mathbb{Z}^{d}$ such that

$$
\begin{gathered}
\mu_{\Lambda, \text { free }}^{\mathrm{RC}}\left(\mathcal{M}_{\Delta, \mathcal{F}}\right) \geq 1-\epsilon / 2 \\
\mu_{\Lambda, m}^{\mathrm{RC}}\left(\mathcal{M}_{\Delta, \mathcal{F}}\right) \geq 1-\epsilon / 4 \\
-\epsilon / 4 \leq \mu_{\Lambda, m}^{\mathrm{RC}}\left(F_{\Lambda,\left\{\mathcal{F}_{i}\right\}}^{m} \mathbb{1}_{\mathcal{M}_{\Delta, \mathcal{F}}}-Q_{\Delta,\left\{\mathcal{F}_{i}\right\}}^{m} \mathbb{1}_{\mathcal{M}_{\Delta,\left\{\mathcal{F}_{i}\right\}}^{m}} F_{\left\{\mathcal{F}_{i}\right\}}^{\text {free }}\right) \leq \epsilon / 4
\end{gathered}
$$

for any $\Lambda \supset \bar{\Lambda}$ and $\bar{\Delta}_{1} \supset \Delta \supset \bar{\Delta}_{2}$, and any $m$ such that $h_{m}=h_{\max }$. Since both $F_{\left\{\mathcal{F}_{i}\right\}, \Lambda}^{m}$ and $F_{\left\{\mathcal{F}_{i}\right\}}^{\text {free }}$ are bounded by one, this yields

$$
\begin{gathered}
\left|\mu_{\Lambda, \text { free }}^{\mathrm{RC}}\left(F_{\left\{\mathcal{F}_{i}\right\}}^{\text {free }}\right)-\mu_{\Lambda, \text { free }}^{\mathrm{RC}}\left(F_{\left\{\mathcal{F}_{i}\right\}}^{\text {free }} \mathbb{1}_{\mathcal{M}_{\Delta, \mathcal{F}}}\right)\right| \leq \epsilon / 2 \\
\left|\mu_{\Lambda, m}^{\mathrm{RC}}\left(F_{\Lambda,\left\{\mathcal{F}_{i}\right\}}^{m}\right)-\mu_{\Lambda, m}^{\mathrm{RC}}\left(Q_{\Delta,\left\{\mathcal{F}_{i}\right\}}^{m} \mathbb{1}_{\mathcal{M}_{\Delta,\left\{\mathcal{F}_{i}\right\}}^{m}} F_{\left\{\mathcal{F}_{i}\right\}}^{\text {free }}\right)\right| \leq \epsilon / 2 .
\end{gathered}
$$

Now the functions $F_{\left\{\mathcal{F}_{i}\right\}}^{\text {free }} \mathbb{1}_{\mathcal{M}_{\Delta, \mathcal{F}}}$ and $Q_{\Delta,\left\{\mathcal{F}_{i}\right\}}^{m} \mathbb{1}_{\mathcal{M}_{\Delta,\left\{\mathcal{F}_{i}\right\}}^{m}} F_{\left\{\mathcal{F}_{i}\right\}}^{\text {free }}$ are quasilocal by Lemma 7.2 and because $Q_{\Delta,\left\{\mathcal{F}_{i}\right\}}^{m}$ is of finite support. Hence, by Theorem 2.3, the limit $\Lambda \nearrow \mathbb{Z}^{d}$ can be performed on the expectations of these functions. Consequently

$$
\begin{gathered}
\left|\limsup _{\Lambda \nearrow \mathbb{Z}^{d}} \mu_{\Lambda, \text { free }}^{\mathrm{RC}}\left(F_{\left\{\mathcal{F}_{i}\right\}}^{\text {free }}\right)-\liminf _{\Lambda \nearrow \mathbb{Z}^{d}} \mu_{\Lambda, \text { free }}^{\mathrm{RC}}\left(F_{\left\{\mathcal{F}_{i}\right\}}^{\text {free }}\right)\right| \leq \epsilon \\
\left|\limsup _{\Lambda \nearrow \mathbb{Z}^{d}} \mu_{\Lambda, m}^{\mathrm{RC}}\left(F_{\Lambda,\left\{\mathcal{F}_{i}\right\}}^{m}\right)-\liminf _{\Lambda \nearrow \mathbb{Z}^{d}} \mu_{\Lambda, m}^{\mathrm{RC}}\left(F_{\Lambda,\left\{\mathcal{F}_{i}\right\}}^{m}\right)\right| \leq \epsilon .
\end{gathered}
$$

The arbitrariness of $\epsilon$ finishes the claim.

\section{Gibbs Uniqueness and Absence of Percolation}

Before proving Theorem 2.5, we shall first establish three useful claims.

Lemma 8.1 Let $\nu \in \mathcal{G}^{\mathrm{ES}}$ be a measure with $\nu(|S| \leq 1)=1$, and let $\mu$ be its $R C$ marginal. Then $\mu \in \mathcal{G}^{\mathrm{RC}}$.

Proof. It suffices to show that for all finite sets of bonds $\mathbb{B}$ and all $\mathbb{B}$-cylinder function $f$, we have $\mu\left(f \mid \boldsymbol{\eta}_{\mathbb{B}^{c}}\right)=\mu_{\mathbb{B}}^{R C}\left(f \mid \boldsymbol{\eta}_{\mathbb{B}^{c}}\right)$. Since $\mu$ is the $\boldsymbol{\eta}$-marginal of $\nu$, it is enough to show that $\nu\left(f \mid \boldsymbol{\eta}_{\mathbb{B}^{c}}\right)=\mu_{\mathbb{B}}^{\mathrm{RC}}\left(f \mid \boldsymbol{\eta}_{\mathbb{B}^{c}}\right)$. By the definition of conditional probabilities, we have that $\nu$-almost surely

$$
\nu\left(f \mid \boldsymbol{\eta}_{\mathbb{B}^{\mathrm{c}}}\right)=\int \nu\left(\mathrm{d} \boldsymbol{\sigma}, \mathrm{d} \boldsymbol{\eta}_{\mathbb{B}} \mid \boldsymbol{\eta}_{\mathbb{B}^{c}}\right) \nu\left(f \mid \boldsymbol{\sigma}_{\Delta^{\mathrm{c}}}, \boldsymbol{\eta}_{\mathbb{B}^{\mathrm{c}}}\right),
$$


for all finite $\Delta$ with $\mathbb{V}(\mathbb{B}) \subset \Delta \subset \mathbb{Z}^{d}$. Given $\boldsymbol{\eta}_{\mathbb{B}^{c}}$, we now take $\Delta$ large enough such that there is no finite cluster $C\left(\boldsymbol{\eta}_{\mathbb{B}^{\mathrm{c}}}\right)$ connecting $\mathbb{V}(\mathbb{B})$ to $\Delta^{\mathrm{c}}$. With this choice, however, one easily computes that $\nu\left(f \mid \boldsymbol{\sigma}_{\Delta^{\mathrm{c}}}, \boldsymbol{\eta}_{\mathbb{B}^{\mathrm{c}}}\right)=\mu_{\mathbb{B}}^{\mathrm{RC}}\left(f \mid \boldsymbol{\eta}_{\mathbb{B}^{\mathrm{c}}}\right)$ for any $\mathbb{B}$-cylinder function $f$, because by the assumption of the lemma, all infinite clusters have almost surely the same color. Since $\mathbb{B}$ is arbitrary, this implies $\mu \in \mathcal{G}^{\mathrm{RC}}$ and, in fact, it implies the stronger statement (3.16).

Lemma 8.2 The measures $\mu_{\mathrm{maxwir}}^{\mathrm{GRC}}$ and $\mu_{\mathrm{free}}^{\mathrm{GRC}}$ are strongly mixing and, in particular, ergodic w.r.t. translations in any of the lattice principal directions.

Proof. Let $\tau$ denote the translation in one of the lattice principal directions. We shall first show that $\mu_{\text {maxwir }}^{\mathrm{GRC}}\left(f g \circ \tau^{n}\right) \rightarrow \mu_{\text {maxwir }}^{\mathrm{GRC}}(f) \mu_{\text {maxwir }}^{\mathrm{GRC}}(g)$ for all $L^{2}$-functions $f$ and $g$. As is well known, it actually suffices to verify this for cylinder functions (which are dense in $L^{2}$ ) and, since we have a space with a natural ordering, we can even restrict ourselves to $f, g$ monotone.

Let $\Delta \subset \mathbb{Z}^{d}$ be a finite set with connected complement $\Delta^{\mathrm{c}}$, and let $f, g$ be non-negative monotone increasing cylinder functions supported in $\mathbb{B}(\Delta)$. Let further $g_{n}=g \circ \tau^{n}$ and $\Delta_{n}=\tau^{n}(\Delta)$. Then $f g_{n}$ is also monotone increasing and hence for any integer $n$ such that $\mathbb{B}(\Delta) \cap \mathbb{B}\left(\Delta_{n}\right)=\emptyset$ and any $\Lambda \supset \Delta \cup \Delta_{n}$, we have

$$
\begin{aligned}
& \mu_{\Lambda, \text { maxwir }}^{\mathrm{GRC}}\left(f g_{n}\right) \leq \mu_{\Lambda, \text { maxwir }}^{\mathrm{GRC}}\left(f g_{n} \mid\left\{\boldsymbol{\eta}_{\mathbb{B}(\Lambda) \backslash\left(\mathbb{B}(\Delta) \cup \mathbb{B}\left(\Delta_{n}\right)\right)}=1\right\}\right)= \\
& =\mu_{\Delta, \text { maxwir }}^{\mathrm{GRC}}(f) \mu_{\Delta_{n}, \text { maxwir }}^{\mathrm{GRC}}\left(g_{n}\right)=\mu_{\Delta \text {,maxwir }}^{\mathrm{GRC}}(f) \mu_{\Delta, \text { maxwir }}^{\mathrm{GRC}}(g) .
\end{aligned}
$$

Taking the limit $\Lambda \nearrow \mathbb{Z}^{d}$ followed by $n \rightarrow \infty$ and $\Delta \nearrow \mathbb{Z}^{d}$, we get

$$
\limsup _{n \rightarrow \infty} \mu_{\operatorname{maxwir}}^{\mathrm{GRC}}\left(f g \circ \tau^{n}\right) \leq \mu_{\operatorname{maxwir}}^{\mathrm{GRC}}(f) \mu_{\operatorname{maxwir}}^{\mathrm{GRC}}(g) .
$$

Since the complementary inequality follows from FKG, the strong mixing property of $\mu_{\text {maxwir }}^{\mathrm{GRC}}$ is established.

The case of the free measure is completely analogous; one just needs to take $f$ and $g$ positive decreasing.

To formulate the next lemma, we need some notation. For a finite connected cluster $C$ of configuration $\boldsymbol{\eta}$ we define a measure $\pi_{C}$ on spin configurations on $\mathbb{V}(C)$ by

$$
\pi_{C}\left(\boldsymbol{\sigma}_{\mathbb{V}(C)}\right)=\sum_{m} \frac{1}{\Theta_{\text {free }}(C)} e^{-\beta h_{m}|\mathbb{V}(C)|} \prod_{x \in \mathbb{V}(C)} \delta_{\sigma_{x}, m} .
$$

For each $m \in Q_{\max }(h)$ we also define an infinite volume coloring measure

$$
\nu_{m}(\boldsymbol{\sigma} \mid \boldsymbol{\eta})=\prod_{C(\boldsymbol{\eta}):|\mathbb{V}(C(\boldsymbol{\eta}))|<\infty} \pi_{C(\boldsymbol{\eta})}\left(\boldsymbol{\sigma}_{\mathbb{V}(C(\boldsymbol{\eta}))}\right) \prod_{x \leftrightarrow \infty} \delta_{\sigma_{x}, m}
$$

Lemma 8.3 Let $m \in Q_{\max }(h)$, and let $\nu \in \mathcal{G}^{\mathrm{ES}}$ with $\nu(S \subseteq\{m\})=1$. Let $\mu$ be the $R C$ marginal of $\nu$. Then for each cylinder function $f$ of $\boldsymbol{\sigma}$ and $\boldsymbol{\eta}$

$$
\nu(f)=\int \mu(\mathrm{d} \boldsymbol{\eta}) \nu_{m}(f \mid \boldsymbol{\eta}) .
$$

In particular, if $\nu_{1}, \nu_{2} \in \mathcal{G}_{1, m}^{\mathrm{ES}}$ are two measures with the same $R C$ marginal, then $\nu_{1}=\nu_{2}$.

Proof. Let $f$ by a $(\Lambda, \mathbb{B}(\Lambda))$ cylinder function. Invoking the argument after $(8.1)$ with $\mathbb{B}=\emptyset$, for $\nu$-almost all $\boldsymbol{\eta}$ (those whose infinite cluster(s) have color $m$ ), we can find $\Delta \supset \Lambda$ large 
enough but finite such that $\nu\left(f \mid \boldsymbol{\sigma}_{\Delta^{\mathrm{c}}}, \boldsymbol{\eta}\right)$ does not depend on $\boldsymbol{\sigma}_{\Delta^{\mathrm{c}}}$, in which case one easily verifies that

$$
\nu\left(f \mid \boldsymbol{\sigma}_{\Delta^{\mathrm{c}}}, \boldsymbol{\eta}\right)=\nu_{m}(f \mid \boldsymbol{\eta}) .
$$

The latter expectation depends only on $\boldsymbol{\eta}$, hence (8.1) implies the desired representation of $\nu(f)$ in terms of $\nu_{m}(f \mid \boldsymbol{\eta})$ and the RC marginal of $\nu$.

Proof of Theorem 2.5(i). We shall prove that any $\nu \in \mathcal{G}^{\mathrm{ES}}$ not exhibiting percolation is equal to the limiting measure $\mu_{\text {free }}^{\mathrm{ES}}$ whose existence was established previously. The proof of this claim goes along the lines of the argument in (5.12)-(5.25), however, it is much simpler in this case due to the absence of infinite clusters.

Let the sequences $\left(\Delta_{n}\right)$ and $\left(\Lambda_{n}(\boldsymbol{\eta})\right)$ be defined as in (5.12). Since there are no infinite components $\nu$-a.s, we have $\Lambda_{n}(\boldsymbol{\eta})=\left\{x \in \Delta_{n}: x \leftrightarrow \Delta_{n}^{\mathrm{c}}\right\}$ and $B_{n}(\boldsymbol{\eta})=\emptyset$ for all $n \geq 1$ and $\nu$-almost all $\boldsymbol{\eta}$. Assume $f$ is a cylinder function and given $\epsilon>0$, take $\Delta$ large enough so that $f$ is supported in $\left(\Delta, \mathbb{B}_{0}(\Delta)\right)$ and

$$
\left|\mu_{V, \text { free }}^{\mathrm{ES}}(f)-\mu_{\text {free }}^{\mathrm{ES}}(f)\right| \leq \epsilon
$$

for all $V \supset \Delta$. Since the indicator function of the event $\left\{\Lambda_{n}(\cdot)=\bar{\Lambda}_{n}\right\}$ does not depend on the configuration in $\left(\Delta, \mathbb{B}_{0}(\Delta)\right)$, we have that

$$
\nu(f)=\nu\left(f \mathbb{1}_{\left\{\Lambda_{n}(\cdot) \not \supset \Delta\right\}}\right)+\sum_{\bar{\Lambda}_{n} \supset \Delta} \nu\left(\mu_{\bar{\Lambda}_{n}, \text { free }}^{\mathrm{ES}}(f) \mathbb{1}_{\left\{\Lambda_{n}(\cdot)=\bar{\Lambda}_{n}\right\}}\right)
$$

by (2.7). Combined with (8.8), this gives the estimate

$$
\begin{aligned}
\nu\left(f \mathbb{1}_{\left\{\Lambda_{n}(\cdot) \not \supset \Delta\right\}}\right)+\left[\mu_{\text {free }}^{\mathrm{ES}}(f)-\epsilon\right] \nu\left(\mathbb{1}_{\left\{\Lambda_{n}(\cdot) \supset \Delta\right\}}\right) \leq \nu(f) \\
\quad \leq \nu\left(f \mathbb{1}_{\left\{\Lambda_{n}(\cdot) \not \supset \Delta\right\}}\right)+\left[\mu_{\text {free }}^{\mathrm{ES}}(f)+\epsilon\right] \nu\left(\mathbb{1}_{\left\{\Lambda_{n}(\cdot) \supset \Delta\right\}}\right) .
\end{aligned}
$$

Since $f$ is bounded and $\Lambda_{n} \nearrow \mathbb{Z}^{d} \nu$-a.s., the bounded convergence theorem yields

$$
\left|\nu(f)-\mu_{\text {free }}^{\mathrm{ES}}(f)\right| \leq \epsilon .
$$

The arbitrariness of $\epsilon$ finishes the claim.

Proof of Theorem 2.5(ii). If $P_{\infty}(\beta, J, h)=0$, then $\mu_{\text {maxwir }}^{\mathrm{ES}}\left(N_{\infty}>0\right)=\mu_{\text {maxwir }}^{\mathrm{RC}}\left(N_{\infty}>0\right)=0$ and (3.14) implies the same is true for any $\nu \in \mathcal{G}^{\mathrm{ES}}$. Thus $\mathcal{G}^{\mathrm{ES}}=\mathcal{G}_{0}^{\mathrm{ES}}=\left\{\mu_{\text {free }}^{\mathrm{ES}}\right\}$. On the other hand, $\mu_{\text {maxwir }}^{\mathrm{RC}}\left(N_{\infty}>0\right)=0$ implies that the same is true for all $\mu \in \mathcal{G}^{\mathrm{RC}}$ by (3.10). Repeating the argument in the proof of Theorem 2.5(i) for the RC measure $\mu$ (and using Theorem 3.4 to guarantee the analogue of $(2.7))$, we get that $\mu=\mu_{\text {free }}^{\mathrm{RC}}$ for all RC Gibbs measures $\mu$, implying $\mathcal{G}^{\mathrm{RC}}=\left\{\mu_{\text {free }}^{\mathrm{RC}}\right\}$.

Remark. Given Theorem 3.4, which is stated for the more general GRC model, the second part of the above proof remains valid for the GRC model. As a consequence, all GRC Gibbs states are equal to the measure $\mu_{\text {free }}^{\mathrm{GRC}}$ if $P_{\infty}(\beta, J, h)=0$, implying that $\mathcal{G}^{\mathrm{GRC}}=\left\{\mu_{\text {free }}^{\mathrm{GRC}}\right\}$ whenever $P_{\infty}(\beta, J, h)=0$.

Proof of Theorem 2.5(iii). We first show that

$$
\mu_{m}^{\mathrm{ES}}\left(\sigma_{x}=\widetilde{m} \mid x \leftrightarrow \infty\right)=\delta_{m, \widetilde{m}}
$$

provided $P_{\infty}(\beta, J, h)>0$ and $m \in Q_{\max }$. Since $\mu_{m}^{\mathrm{ES}}\left(N_{\infty}=1\right)=1$ if $P_{\infty}(\beta, J, h)>0$, equation (8.12) implies that $\mu_{m}^{\mathrm{ES}}\left(\mathcal{A}_{1, m}^{\infty}\right)=1$. 
To prove (8.12), we recall the well known fact that

$$
\mu_{\text {maxwir }}^{\mathrm{RC}}(0 \leftrightarrow \infty)=\lim _{\Lambda \nearrow \mathbb{Z}^{d}} \mu_{\Lambda, \text { maxwir }}^{\mathrm{RC}}\left(0 \leftrightarrow \Lambda^{\mathrm{c}}\right),
$$

see equation (7.19) above. As a consequence, we get that for all $m \in Q_{\max }$,

$$
\mu_{m}^{\mathrm{ES}}(x \leftrightarrow \infty)=\lim _{\Lambda \nearrow \mathbb{Z}^{d}} \mu_{\Lambda, m}^{\mathrm{ES}}\left(0 \leftrightarrow \Lambda^{\mathrm{c}}\right) .
$$

Combined with the fact that $\mu_{\Lambda, m}^{\mathrm{ES}}\left(0 \leftrightarrow \Lambda^{\mathrm{c}}, \sigma_{x}=\widetilde{m}\right)=\mu_{\Lambda, m}^{\mathrm{ES}}\left(0 \leftrightarrow \Lambda^{\mathrm{c}}\right) \delta_{m, \widetilde{m}}$, this implies (8.12). It remains to show that the state $\mu_{m}^{\mathrm{ES}}$ is extremal whenever $m \in Q_{\max }$. To this end, let us assume that

$$
\mu_{m}^{\mathrm{ES}}=\lambda \nu_{1}+(1-\lambda) \nu_{2}
$$

with $\nu_{i}\left(\mathcal{A}_{1, m}^{\infty}\right)=1$ and $0<\lambda<1$. By Lemma 8.1, the RC marginals $\mu_{i}$ of $\nu_{i}$ are RC Gibbs states, which implies that (8.15) induces a similar decomposition for $\mu_{\text {maxwir }}^{\mathrm{RC}}$. However, $\mu_{\operatorname{maxwir}}^{\mathrm{RC}}$ is extremal by Lemma 8.2, which implies that $\mu_{1}=\mu_{2}=\mu_{\text {maxwir }}^{\mathrm{RC}}$. Using Lemma 8.3, this implies $\nu_{1}=\nu_{2}$, and hence extremality of $\mu_{m}^{\mathrm{ES}}$.

\section{Random Cluster Gibbs Measures for $d=2$}

Proof of Theorem 2.6(i). The proof of Theorem 2.6(i) remains again valid for the more general GRC model. For $J<J_{c}$, the statement has already been proven in the last section. Let us therefore suppose that $J>J_{c}$ and $d=2$. Then the first condition (and item (iii) of Corollary of Theorem 3.1) implies that there is percolation under $\mu_{\text {maxwir }}^{\mathrm{GRC},}$. Moreover, since $\mu_{\text {maxwir }}^{\mathrm{GRC}, J}$ satisfies the following claims

(1) $\mu_{\text {maxwir }}^{\mathrm{GRC} J}$ is separately ergodic in all lattice directions

(2) $\mu_{\text {maxwir }}^{\mathrm{GRC}}$ is invariant under lattice reflections and rotations

(3) $\mu_{\text {maxwir }}^{\mathrm{GRC} J}$ is FKG,

as has been proved previously, the powerful result of [14] asserts that the infinite cluster is unique under $\mu_{\text {maxwir }}^{\mathrm{GRC}}$. Moreover, by a corollary to this result, the cluster contains an infinite series of nested circuits that (eventually) encircle any point of the lattice.

Now, according to Theorem 3.1(iii), any $\mu \in \mathcal{G}^{\text {GRC }}$ at the coupling constant $J$ is FKG dominating the measure $\mu_{\text {free }}^{\mathrm{GRC},}$. Let $J>J_{1}>J_{c}$. Then

$$
\mu(\cdot) \underset{\mathrm{FKG}}{\geq} \mu_{\text {free }}^{\mathrm{GRC}, J}(\cdot) \underset{\mathrm{FKG}}{\geq} \mu_{\text {maxwir }}^{\mathrm{GRC}, J_{1}}(\cdot),
$$

where the second inequality is Theorem 3.1(iv). Thus, all GRC Gibbs measures at $J$ exhibit an infinite cluster as well as the above circuits about the origin, because the latter is an FKG increasing event.

The proof is concluded in a manner similar to the argument (8.8)-(8.11). Let thus $f$ be a cylinder function with support in $\mathbb{B}(\Delta)$, where $\Delta$ is supposed to be sufficiently large so that

$$
\left|\mu_{V, \text { maxwir }}^{\mathrm{GRC}}(f)-\mu_{\operatorname{maxwir}}^{\mathrm{GRC}}(f)\right| \leq \epsilon
$$

for any $V \supset \Delta$. Let $\left\{\Delta_{n}\right\}$ be an increasing sequence of boxes centered at the origin, and let $\Omega_{n}$ be the set of all configurations $\boldsymbol{\eta}$ for which there exists a closed circuit $\Gamma$ of occupied bonds surrounding $\Delta$ and connected to the infinite cluster, such that it is entirely contained in $\mathbb{B}_{0}\left(\Delta_{n}\right)$. Let us use $\Gamma_{n}(\boldsymbol{\eta})$ to denote the outermost such circuit contained in $\mathbb{B}_{0}\left(\Delta_{n}\right)$ and $V_{\Gamma_{n}}(\boldsymbol{\eta})$ the set of its interior sites. Let $\mathbb{1}_{\Omega_{n}}$ be the characteristic function of $\Omega_{n}$ and, for a 
given circuit $\Gamma$, let $\mathbb{1}_{\left\{\Gamma_{n}=\Gamma\right\}}$ denote the characteristic function of the set of all configurations such that the corresponding outermost circuit $\Gamma_{n}$ equals $\Gamma$.

Using the fact that the function $\mathbb{1}_{\left\{\Gamma_{n}=\Gamma\right\}}$ does not depend on the values of the GRC configuration on $\mathbb{B}\left(V_{\Gamma}\right)$, we now apply Theorem 3.4 with $\mathbb{B}=\mathbb{B}\left(V_{\Gamma}\right)$ to get

$$
\begin{aligned}
\mu\left(f \mathbb{1}_{\Omega_{n}}\right)=\sum_{\Gamma} \mu\left(f \mathbb{1}_{\left\{\Gamma_{n}=\Gamma\right\}}\right)=\sum_{\Gamma} \mu\left(\mu_{\mathbb{B}\left(V_{\Gamma}\right)}\left(f \mid \boldsymbol{\eta}_{\mathbb{B}\left(V_{\Gamma}\right)^{\mathrm{c}}}\right)\right. & \left.\mathbb{1}_{\left\{\Gamma_{n}=\Gamma\right\}}\right) \\
& =\sum_{\Gamma} \mu\left(\mu_{V_{\Gamma}, \operatorname{maxwir}}(f) \mathbb{1}_{\left\{\Gamma_{n}=\Gamma\right\}}\right),
\end{aligned}
$$

where the sum is over all closed circuits $\Gamma$ of occupied bonds surrounding $\Delta$ and contained wholly in $\mathbb{B}_{0}\left(\Delta_{n}\right)$. Since $f$ is bounded and $\mu\left(\Omega_{n}\right) \rightarrow 1$ as $n \rightarrow \infty$, we have that $\mid \mu\left(f \mathbb{1}_{\Omega_{n}}\right)-$ $\mu(f) \mid \leq \epsilon$ for $n$ sufficiently large. Using (9.2) for $V=V_{\Gamma}$, we conclude that

$$
\left|\mu(f)-\mu_{\text {maxwir }}^{\mathrm{GRC}}(f)\right| \leq 3 \epsilon \text {. }
$$

Since $\epsilon$ is arbitrary, we get the desired statement that each GRC Gibbs state necessarily equals the measure $\mu_{\text {maxwir }}^{\mathrm{GRC}}$.

Proof of Theorem 2.6(ii). We again only need to prove the statement of $J>J_{c}$. Using Theorem 3.2, equation (3.15) instead of Theorem 3.1(iii), we obtain the bound (9.1) for the $\mathrm{RC}$ marginal $\mu$ of any $\nu \in \mathcal{G}^{\mathrm{ES}}$ with $\left|Q_{\max }\right|=1$. Let $\nu$ be such a measure. Applying the steps leading to (9.3) to the measure $\nu$ and a cylinder function $f$ with support in $(\Delta, \mathbb{B}(\Delta))$, we will have to calculate the conditional expectation $\nu\left(f \mid \boldsymbol{\sigma}_{V_{\Gamma}^{\mathrm{c}}}, \boldsymbol{\eta}_{\mathbb{B}\left(V_{\Gamma}\right)^{\mathrm{c}}}\right)=\mu_{V_{\Gamma}, \mathbb{B}\left(V_{\Gamma}\right)}^{\mathrm{ES}}\left(f \mid \boldsymbol{\sigma}_{V_{\Gamma}^{\mathrm{c}}}, \boldsymbol{\eta}_{\mathbb{B}\left(V_{\Gamma}\right)^{\mathrm{c}}}\right)$. By Theorem 5.1 the value of $\sigma_{x}$ on the sites $x \in \partial V_{\Gamma}$ is constrained to be one of the colors in $Q_{\text {max }}$. Since we assumed that $\left|Q_{\max }\right|=1$, we obtain that $\nu\left(f \mid \boldsymbol{\sigma}_{V_{\Gamma}^{\mathrm{c}}}, \boldsymbol{\eta}_{\mathbb{B}\left(V_{\Gamma}\right)^{\mathrm{c}}}\right)=\mu_{V_{\Gamma}, m}^{\mathrm{ES}}(f)$, where $m$ is the unique spin for which $h_{m}=h_{\max }$. Continuing as in the proof of (i), we obtain that $\nu=\mu_{m}^{\mathrm{ES}}$.

\section{Maps between ES, Spin and RC Gibbs Measures}

Proof of Theorem 2.1. Let $\mu_{\Lambda}^{\text {SPIN }}\left(\cdot \mid \sigma_{\Lambda^{\mathrm{c}}}\right)$ denote the Gibbs measure on spins in $\Lambda$ with boundary condition $\sigma_{\Lambda^{\mathrm{c}}}$. The proof is based on the crucial observations that, for the special choice $\mathbb{B}=\mathbb{B}(\Lambda)$,

(A) $\mu_{\Lambda, \mathbb{B}(\Lambda)}^{\mathrm{ES}}\left(\cdot \mid \boldsymbol{\sigma}_{\Lambda^{\mathrm{c}}}, \boldsymbol{\eta}_{\mathbb{B}(\Lambda)^{\mathrm{c}}}\right)$ does not depend on $\boldsymbol{\eta}_{\mathbb{B}(\Lambda)^{\mathrm{c}}}$.

(B) The spin marginal of $\mu_{\Lambda, \mathbb{B}(\Lambda)}^{\mathrm{ES}}\left(\cdot \mid \boldsymbol{\sigma}_{\Lambda^{\mathrm{c}}}, \boldsymbol{\eta}_{\mathbb{B}(\Lambda)^{\mathrm{c}}}\right)$ is precisely $\mu_{\Lambda}^{\mathrm{sPN}}\left(\cdot \mid \boldsymbol{\sigma}_{\Lambda^{\mathrm{c}}}\right)$.

Let now $\nu \in \mathcal{G}^{\mathrm{ES}}, \Lambda \subset \mathbb{Z}^{d}$ be finite, and let $f$ be a function depending only on the spin configuration in $\Lambda$. Then, by (2.6), (A), (B), and the definition of marginals, we have

$$
\begin{aligned}
\left(\Pi_{S} \nu\right)(f)=\nu(f)=\int \nu(\mathrm{d} \boldsymbol{\sigma}, \mathrm{d} \boldsymbol{\eta}) \mu_{\Lambda, \mathbb{B}(\Lambda)}^{\mathrm{ES}}\left(f \mid \boldsymbol{\sigma}_{\Lambda^{\mathrm{c}}}, \boldsymbol{\eta}_{\mathbb{B}(\Lambda)^{\mathrm{c}}}\right) & = \\
=\int \nu(\mathrm{d} \boldsymbol{\sigma}, \mathrm{d} \boldsymbol{\eta}) \mu_{\Lambda}^{\mathrm{SPIN}}\left(f \mid \boldsymbol{\sigma}_{\Lambda^{\mathrm{c}}}\right) & =\int\left(\Pi_{S} \nu\right)(\mathrm{d} \boldsymbol{\sigma}) \mu_{\Lambda}^{\mathrm{SPN}}\left(f \mid \boldsymbol{\sigma}_{\Lambda^{\mathrm{c}}}\right),
\end{aligned}
$$

proving that $\Pi_{S} \nu \in \mathcal{G}^{\text {SPIN }}$. Hence, indeed, $\Pi_{S}$ is a map from $\mathcal{G}^{\mathrm{ES}}$ to $\mathcal{G}^{\text {SPIN }}$.

To prove that $\Pi_{S}$ is an isomorphism, let us first establish its surjectivity. We begin by noting that the set $\{(\Lambda, \mathbb{B}(\Lambda))\}$ is cofinal in the set of all pairs $\{(\Lambda, \mathbb{B})\}$, ordered by inclusion. (Namely, for any $(\Lambda, \mathbb{B})$ there exist $\bar{\Lambda}$ such that $\Lambda \subset \bar{\Lambda}$ and $\mathbb{B} \subset \mathbb{B}(\bar{\Lambda})$.) Then it is easy to 
see that the validity of $(2.6)$ for the pairs $(\Lambda, \mathbb{B}(\Lambda))$ implies its validity for general $(\Lambda, \mathbb{B})$ (see Remark 1.24 of $[15])$. Let now $\mu \in \mathcal{G}^{\text {SPIN }}$ and consider the following ES measure

$$
\nu_{\Lambda}(\cdot)=\int \mu(\mathrm{d} \boldsymbol{\sigma}) \mu_{\Lambda, \mathbb{B}(\Lambda)}^{\mathrm{ES}}\left(\cdot \mid \boldsymbol{\sigma}_{\Lambda^{\mathrm{c}}}, \boldsymbol{\eta}_{\mathbb{B}(\Lambda)^{\mathrm{c}}}\right)
$$

on the set of on configurations in $(\Lambda, \mathbb{B}(\Lambda))$. Here the configuration $\boldsymbol{\eta}_{\mathbb{B}(\Lambda)^{c}}$ is added only for the formal completeness since by (A) its value does not matter for $\nu_{\Lambda}$. By taking into account the consistency of the finite volume ES measures $\left\{\mu_{\Lambda, \mathbb{B}}^{\mathrm{ES}}\right\}$, the measures $\nu_{\Lambda}(\cdot)$ satisfy the restricted DLR equations

$$
\nu_{\Lambda}(f)=\int \nu_{\Lambda}(\mathrm{d} \boldsymbol{\sigma}, \mathrm{d} \boldsymbol{\eta}) \mu_{\widetilde{\Lambda}, \widetilde{\mathbb{B}}}^{\mathrm{ES}}\left(f \mid \boldsymbol{\sigma}_{\widetilde{\Lambda}^{c}}, \boldsymbol{\eta}_{\widetilde{\mathbb{B}}^{c}}\right)
$$

for any $\widetilde{\Lambda} \subset \Lambda, \widetilde{\mathbb{B}} \subset \mathbb{B}(\Lambda)$, and any $\widetilde{\Lambda}, \widetilde{B}$-cylinder function $f$. Moreover, let $\Lambda_{1} \supset \Lambda_{2} \supset \widetilde{\Lambda}$ be two sets. Then for any such function $f$ (as before) we have

$$
\begin{aligned}
\nu_{\Lambda_{1}}(f) & =\int \mu(\mathrm{d} \boldsymbol{\sigma}) \mu_{\Lambda_{1}, \mathbb{B}\left(\Lambda_{1}\right)}^{\mathrm{ES}}\left(f \mid \boldsymbol{\sigma}_{\Lambda_{1}^{\mathrm{c}}}, \boldsymbol{\eta}_{\mathbb{B}\left(\Lambda_{1}\right)^{\mathrm{c}}}\right) \\
& =\int \mu(\mathrm{d} \boldsymbol{\sigma}) \mu_{\Lambda_{1}, \mathbb{B}\left(\Lambda_{1}\right)}^{\mathrm{ES}}\left(\mu_{\Lambda_{2}, \mathbb{B}\left(\Lambda_{2}\right)}^{\mathrm{ES}}(f \mid \cdot) \mid \boldsymbol{\sigma}_{\Lambda_{1}^{\mathrm{c}}}, \boldsymbol{\eta}_{\mathbb{B}\left(\Lambda_{1}\right)^{\mathrm{c}}}\right) \\
& =\int \mu(\mathrm{d} \boldsymbol{\sigma}) \mu_{\Lambda_{1}}^{\mathrm{SPIN}}\left(\mu_{\Lambda_{2}, \mathbb{B}\left(\Lambda_{2}\right)}^{\mathrm{ES}}(f \mid \cdot) \mid \boldsymbol{\sigma}_{\Lambda_{1}^{\mathrm{c}}}\right) \\
& =\int \mu(\mathrm{d} \boldsymbol{\sigma}) \mu_{\Lambda_{2}, \mathbb{B}\left(\Lambda_{2}\right)}^{\mathrm{ES}}\left(f \mid \boldsymbol{\sigma}_{\Lambda_{2}^{\mathrm{c}}}, \boldsymbol{\eta}_{\mathbb{B}\left(\Lambda_{2}\right)^{\mathrm{c}}}\right)=\nu_{\Lambda_{2}}(f) .
\end{aligned}
$$

Here the first equality is due to (10.2), the second one follows from the fact that $\mu_{\Lambda_{1}, \mathbb{B}\left(\Lambda_{1}\right)}^{\mathrm{ES}}$ is a finite volume Gibbs measure, the third one is established by applying (A) to the measure $\mu_{\Lambda_{2}, \mathbb{B}\left(\Lambda_{2}\right)}^{\mathrm{ES}}(f \mid \cdot)$ and subsequently $(\mathrm{B})$ to the expectation w.r.t. $\mu_{\Lambda_{1}, \mathbb{B}\left(\Lambda_{1}\right)}^{\mathrm{ES}}$, and, finally, the fourth equality follows from the fact that $\mu \in \mathcal{G}^{\text {SPIN }}$. Consequently, as $\Lambda \nearrow \mathbb{Z}^{d}, \nu_{\Lambda}(f)$ is eventually a constant for any cylinder function $f$. In particular, the weak limit $\nu=\lim _{\Lambda} \mathbb{Z}^{d} \nu_{\Lambda}$ exists and, by (10.3), it satisfies (2.6), i.e., $\nu \in \mathcal{G}^{\mathrm{ES}}$. Finally, $\Pi_{S} \nu=\mu$, since for any $\Lambda$-cylinder function $f$ of spins

$$
\left(\Pi_{S} \nu\right)(f)=\nu(f)=\int \mu(\mathrm{d} \boldsymbol{\sigma}) \mu_{\Lambda, \mathbb{B}(\Lambda)}^{\mathrm{ES}}\left(f \mid \boldsymbol{\sigma}_{\Lambda^{\mathrm{c}}}, \boldsymbol{\eta}_{\mathbb{B}(\Lambda)^{\mathrm{c}}}\right)=\int \mu(\mathrm{d} \boldsymbol{\sigma}) \mu_{\Lambda}^{\mathrm{SPIN}}\left(f \mid \boldsymbol{\sigma}_{\Lambda^{\mathrm{c}}}\right)=\mu(f),
$$

proving that $\Pi_{S}$ is surjective.

In order to see that $\Pi_{S}$ is also injective, we notice that if $\widetilde{\nu} \in \mathcal{G}^{\mathrm{ES}}$ is such that $\Pi_{S} \widetilde{\nu}=\mu$, then

$$
\widetilde{\nu}(f)=\widetilde{\nu}\left(\mu_{\Lambda, \mathbb{B}(\Lambda)}^{\mathrm{ES}}(f \mid \cdot)\right)=\left(\Pi_{S} \widetilde{\nu}\right)\left(\mu_{\Lambda, \mathbb{B}(\Lambda)}^{\mathrm{ES}}(f \mid \cdot)\right)=\mu\left(\mu_{\Lambda, \mathbb{B}(\Lambda)}^{\mathrm{ES}}(f \mid \cdot)\right)
$$

for any $(\Lambda, \mathbb{B}(\Lambda))$-cylinder function $f$. Here the first equation is the DLR equation for $\widetilde{\nu}$, the second equation follows from (A), and the third equation is the assumption $\Pi_{S} \widetilde{\nu}=\mu$. Now, the right hand sides of (10.6) and (10.2) coincide, so $\widetilde{\nu}=\nu$, with $\nu$ defined by taking the limit $\Lambda \nearrow \mathbb{Z}^{d}$ of $\nu_{\Lambda}$ in (10.2). In particular, all measures $\widetilde{\nu}$ satisfying $\Pi_{S} \widetilde{\nu}=\mu$ are equal, yielding thus injectivity of $\Pi_{S}$.

The part of the claim concerning translation invariant measures is proved in the same way, because both constructions (10.1) and (10.2) preserve translation invariance. 
Proof of Theorem 2.2(i). We first note that the marginal of any ES Gibbs state with at most one infinite cluster is an RC Gibbs state by Lemma 8.1. This proves that $\Pi_{\mathrm{RC}}$ maps $\mathcal{G}_{\leq 1}^{\mathrm{ES}}$ into $\mathcal{G}^{\mathrm{RC}}$.

Next we show that the map is surjective on $\mathcal{G}_{\leq 1}^{\mathrm{RC}}$. Let $\mu \in \mathcal{G}_{\leq 1}^{\mathrm{RC}}$. Recall the definition of $F_{\left\{\mathcal{F}_{i}\right\}}^{\text {free }}$ in (7.1). It turns out that $F_{\left\{\mathcal{F}_{i}\right\}}^{\text {free }}$ satisfies the following identity:

$$
\sum_{m=1}^{q} F_{\left\{\mathcal{F}_{1}, \ldots, \mathcal{F}_{m-1}, \mathcal{F}_{m} \cup\{x\}, \mathcal{F}_{m+1}, \ldots, \mathcal{F}_{q}\right\}}^{\text {free }}(\boldsymbol{\eta})=F_{\left\{\mathcal{F}_{i}\right\}}^{\text {free }}(\boldsymbol{\eta})
$$

for each $\left\{\mathcal{F}_{i}\right\}$, any $x \notin \mathcal{F}=\cup_{i} \mathcal{F}_{i}$ and any $\boldsymbol{\eta}$. Namely, let $\mathcal{F}_{i} \leftrightarrow \mathcal{F}_{j}$ for $i \neq j$ in $\boldsymbol{\eta}$ and suppose $x \leftrightarrow \mathcal{F}_{m}$ for some $m$. Then the sum on the l.h.s. of (10.7) degenerates to the $m$-th entry, which is easily identified with the r.h.s. On the other hand, if $x \leftrightarrow \mathcal{F}_{m}$ for all $m$, then the sum in (10.7) can be propagated through the products in (7.1) up to the last term, where the desired identity then follows by taking also (3.2) into account.

The relation (10.7) enables us to define a joint measure on $\boldsymbol{\sigma}$ and $\boldsymbol{\eta}$. Let $\mu \in \mathcal{G}^{\mathrm{RC}}$ and let $\mathcal{A}_{\left\{\mathcal{F}_{i}\right\}}$ denote the event

$$
\mathcal{A}_{\left\{\mathcal{F}_{i}\right\}}=\left\{\boldsymbol{\sigma}: \sigma_{x}=m \forall x \in \mathcal{F}_{m}\right\} .
$$

Note that $\mathcal{A}_{\left\{\mathcal{F}_{i}\right\}}$ is a cylinder event in $\mathcal{F}$. Consider the set function $\nu$, for the sets on the product space of configurations $(\boldsymbol{\sigma}, \boldsymbol{\eta})$, defined as

$$
\nu\left(\mathcal{A}_{\left\{\mathcal{F}_{i}\right\}} \times \mathcal{B}\right)=\mu\left(F_{\left\{\mathcal{F}_{i}\right\}}^{\text {free }} \mathbb{1}_{\mathcal{B}}\right),
$$

where $\mathcal{B}$ stands for any cylinder event on configurations $\boldsymbol{\eta}$. Due to the fact that $\mu$ is a measure on $\boldsymbol{\eta}$ and due to (10.7), the set function defined in (10.9) satisfies the consistency condition for all finite volume projections and, by the Kolmogorov theorem, it thus gives rise to a measure on $(\boldsymbol{\sigma}, \boldsymbol{\eta})$.

Using (10.7), the $\boldsymbol{\eta}$-marginal of $\nu$ is $\mu$, so it remains to show that $\nu \in \mathcal{G}^{\mathrm{ES}}$. Due to the consistency of the ES specifications (2.4), it is enough to show that $\nu$-almost surely

$$
\nu\left(\boldsymbol{\sigma}_{\Delta}, \boldsymbol{\eta}_{\mathbb{B}(\Delta)} \mid \boldsymbol{\sigma}_{\Delta^{\mathrm{c}}}, \boldsymbol{\eta}_{\mathbb{B}(\Delta)^{\mathrm{c}}}\right)=\mu_{\Delta, \mathbb{B}(\Delta)}^{\mathrm{ES}}\left(\boldsymbol{\sigma}_{\Delta}, \boldsymbol{\eta}_{\mathbb{B}(\Delta)} \mid \boldsymbol{\sigma}_{\partial \Delta}, \boldsymbol{\eta}_{\mathbb{B}(\Delta)^{\mathrm{c}}}\right)
$$

for all finite $\Delta \subset \mathbb{Z}^{d}$. For that, it actually suffices to establish that

$$
\lim _{\Lambda \nearrow \mathbb{Z}^{d}} \nu\left(\boldsymbol{\sigma}_{\Delta}, \boldsymbol{\eta}_{\mathbb{B}(\Delta)} \mid \boldsymbol{\sigma}_{\Lambda \backslash \Delta}, \boldsymbol{\eta}_{\mathbb{B}(\Delta)^{\mathrm{c}}}\right)=\mu_{\Delta, \mathbb{B}(\Delta)}^{\mathrm{ES}}\left(\boldsymbol{\sigma}_{\Delta}, \boldsymbol{\eta}_{\mathbb{B}(\Delta)} \mid \boldsymbol{\sigma}_{\partial \Delta}, \boldsymbol{\eta}_{\mathbb{B}(\Delta)^{\mathrm{c}}}\right) .
$$

To calculate the l.h.s., we shall evaluate $\nu\left(\boldsymbol{\sigma}_{\Lambda}, \boldsymbol{\eta}_{\mathbb{B}(\Delta)} \mid \boldsymbol{\eta}_{\mathbb{B}(\Delta)^{\mathrm{c}}}\right)$. In order to keep the expressions short, we assume without loss of generality that $h_{\max }=0$. Using (10.9) and the strong form of the DLR equation (3.16), we write

$$
\nu\left(\boldsymbol{\sigma}_{\Lambda}, \boldsymbol{\eta}_{\mathbb{B}(\Delta)} \mid \boldsymbol{\eta}_{\mathbb{B}(\Delta)^{\mathrm{c}}}\right)=\mu\left(F_{\left\{\Lambda_{i}\right\}} \mathbb{1}_{\left\{\boldsymbol{\eta}_{\mathbb{B}(\Delta)}\right\}} \mid \boldsymbol{\eta}_{\mathbb{B}(\Delta)^{\mathrm{c}}}\right)=F_{\left\{\Lambda_{i}\right\}}(\boldsymbol{\eta}) \mu_{\mathbb{B}(\Delta)}^{\mathrm{RC}}\left(\boldsymbol{\eta}_{\mathbb{B}(\Delta)} \mid \boldsymbol{\eta}_{\mathbb{B}(\Delta)^{\mathrm{c}}}\right),
$$

where $\left(\Lambda_{i}\right)$ is the partition of $\Lambda$ defined by $\Lambda_{i}=\left\{x \in \Lambda \mid \sigma_{x}=i\right\}$.

In order to evaluate the r.h.s., we use (7.1) and (3.6) to get

$$
\begin{aligned}
F_{\left\{\Lambda_{i}\right\}}^{\mathrm{free}}(\boldsymbol{\eta}) \mu_{\mathbb{B}(\Delta)}^{\mathrm{RC}}\left(\boldsymbol{\eta}_{\mathbb{B}(\Delta)} \mid \boldsymbol{\eta}_{\mathbb{B}(\Delta)^{\mathrm{c}}}\right) & =\prod_{i<j} \mathbb{1}_{\left\{\Lambda_{i} \leftrightarrow \Lambda_{j}\right\}}(\boldsymbol{\eta}) \prod_{m=1}^{q} \prod_{\mathbb{V}(C(\boldsymbol{\eta})) \cap \Lambda_{m} \neq \emptyset} \frac{e^{\beta h_{m}|\mathbb{V}(C(\boldsymbol{\eta}))|}}{\Theta_{\text {free }}(C(\boldsymbol{\eta}))} \\
& \times \frac{\left(e^{\beta J}-1\right)^{\left|\mathbb{B}_{\mathrm{occ}}(\boldsymbol{\eta}) \cap \mathbb{B}(\Delta)\right|}}{\mathcal{Z}_{\mathbb{B}(\Delta)}\left(\boldsymbol{\eta}_{\left.\mathbb{B}(\Delta)^{\mathrm{c}}\right)}\right.} \prod_{\mathbb{V}(C(\boldsymbol{\eta})) \cap \mathbb{V}(\mathbb{B}(\Delta)) \neq \emptyset} \Theta_{\text {free }}(C(\boldsymbol{\eta})),
\end{aligned}
$$


where $\mathcal{Z}_{\mathbb{B}(\Delta)}\left(\boldsymbol{\eta}_{\mathbb{B}(\Delta)^{\mathrm{c}}}\right)$ is the normalization factor for $\mu_{\mathbb{B}(\Delta)}^{\mathrm{RC}}\left(\cdot \mid \boldsymbol{\eta}_{\mathbb{B}(\Delta)^{\mathrm{c}}}\right)$. Rewriting

$$
\begin{aligned}
\prod_{m=1 \mathbb{V}(C(\boldsymbol{\eta})) \cap \Lambda_{m} \neq \emptyset}^{q} \prod \frac{e^{\beta h_{m}|\mathbb{V}(C(\boldsymbol{\eta}))|}}{\Theta_{\text {free }}(C(\boldsymbol{\eta}))} \times \prod_{\mathbb{V}(C(\boldsymbol{\eta})) \cap \mathbb{V}(\mathbb{B}(\Delta)) \neq \emptyset} \Theta_{\text {free }}(C(\boldsymbol{\eta})) \\
=\prod_{m=1}^{q} \prod_{\substack{\mathbb{V}(C(\boldsymbol{\eta})) \cap \Lambda_{m} \neq \emptyset \\
\mathbb{V}(C(\boldsymbol{\eta})) \cap \Delta=\emptyset}} \frac{e^{\beta h_{m}|\mathbb{V}(C(\boldsymbol{\eta}))|}}{\Theta_{\text {free }}(C(\boldsymbol{\eta}))} \times \prod_{m=1}^{q} \prod_{\substack{\mathbb{V}(C(\boldsymbol{\eta}))\left(\mathbb{V}(\mathbb{B}(\Delta)) \neq \emptyset \\
\mathbb{V}(C(\boldsymbol{\eta})) \cap \mathbb{\Delta}_{m} \neq \emptyset\right.}} e^{\beta h_{m}|\mathbb{V}(C(\boldsymbol{\eta}))|},
\end{aligned}
$$

where we introduced $\bar{\Delta}=\Delta \cup \partial \Delta$ and $\bar{\Delta}_{m}=\Lambda_{m} \cap \bar{\Delta}$, and inserting the identity

$$
e^{\beta h_{m}|\mathbb{V}(C(\boldsymbol{\eta}))|}=e^{\beta h_{m}|\mathbb{V}(C(\boldsymbol{\eta})) \cap \Delta|} e^{\beta h_{m}\left|\mathbb{V}(C(\boldsymbol{\eta})) \cap \Delta^{\mathrm{c}}\right|},
$$

we can now extract all terms that depend on $\boldsymbol{\sigma}_{\Delta}$ and $\boldsymbol{\eta}_{\mathbb{B}(\Delta)}$ from the r.h.s. of (10.13) to obtain the Gibbs factor $W\left(\boldsymbol{\sigma}_{\Delta}, \boldsymbol{\eta}_{\mathbb{B}(\Delta)} \mid \boldsymbol{\sigma}_{\partial \Delta}, \boldsymbol{\eta}_{\mathbb{B}(\Delta)^{\mathrm{c}}}\right)$ times a term depending only on $\boldsymbol{\sigma}_{\Lambda \backslash \Delta}$ and $\boldsymbol{\eta}_{\mathbb{B}(\Delta)^{\mathrm{c}}}$. This yields the representation

$$
\nu\left(\boldsymbol{\sigma}_{\Lambda}, \boldsymbol{\eta}_{\mathbb{B}(\Delta)} \mid \boldsymbol{\eta}_{\mathbb{B}(\Delta)^{\mathrm{c}}}\right)=N\left(\boldsymbol{\sigma}_{\Lambda \backslash \Delta}, \boldsymbol{\eta}_{\mathbb{B}(\Delta)^{\mathrm{c}}}\right) \mu_{\Delta, \mathbb{B}(\Delta)}^{\mathrm{ES}}\left(\boldsymbol{\sigma}_{\Delta}, \boldsymbol{\eta}_{\mathbb{B}(\Delta)} \mid \boldsymbol{\sigma}_{\partial \Delta}, \boldsymbol{\eta}_{\mathbb{B}(\Delta)^{\mathrm{c}}}\right),
$$

which in turn leads to the identity

$$
\nu\left(\boldsymbol{\sigma}_{\Delta}, \boldsymbol{\eta}_{\mathbb{B}(\Delta)} \mid \boldsymbol{\sigma}_{\Lambda \backslash \Delta}, \boldsymbol{\eta}_{\mathbb{B}(\Delta)^{\mathrm{c}}}\right)=\mu_{\Delta, \mathbb{B}(\Delta)}^{\mathrm{ES}}\left(\boldsymbol{\sigma}_{\Delta}, \boldsymbol{\eta}_{\mathbb{B}(\Delta)} \mid \boldsymbol{\sigma}_{\partial \Delta}, \boldsymbol{\eta}_{\mathbb{B}(\Delta)^{\mathrm{c}}}\right),
$$

provided that $\boldsymbol{\sigma}_{\Lambda \backslash \Delta}$ is consistent with $\boldsymbol{\eta}_{\mathbb{B}(\Delta)^{c}}$. Equation (10.17) immediately gives the desired claim (10.11) and hence (10.10).

Proof of Theorem 2.2(ii-iv). Let $\nu \in \mathcal{G}_{\leq 1}^{\mathrm{ES}}$. Since $\left\{N_{\infty}=0\right\}$ is a tail event, there is a unique decomposition of $\nu$ into $\lambda_{0} \nu_{0}+\lambda_{>0} \nu_{>0}$, where $\nu_{0} \in \mathcal{G}_{0}^{\mathrm{ES}}$ and $\nu_{>0} \in \mathcal{G}_{1}^{\mathrm{ES}}$. The decomposition (2.13) then follows by further conditioning upon the color of the spin on the infinite cluster of $\nu_{>0}$. This proves (ii). To prove (iii), we just invoke Theorem 2.5(i), Lemma 8.3 and Theorem 2.2(i). To prove (iv), we need to realize that if $\left|Q_{\max }(h)\right|=1$, then the decomposition is completed already by conditioning on the presence/absence of the infinite cluster, which works the same on both $\mathcal{G}_{\leq 1}^{\mathrm{ES}}$ and $\mathcal{G}_{\leq 1}^{\mathrm{RC}}$.

\section{Acknowledgments}

We thank Ken Alexander for interesting discussions, and Hans-Otto Georgii for pointing out an error in one of our original arguments. M.B. gratefully acknowledges the support of the Fields Institute in Toronto.

\section{REFERENCES}

[1] M. Aizenman, Translation invariance and instability of phase coexistence in the two-dimensional Ising model, Commun. Math. Phys. 73, 83-94 (1980)

[2] M. Aizenman, J.T. Chayes, L. Chayes, C.M. Newman, Discontinuity of the magnetization in onedimensional $1 /|x-y|^{2}$ Ising and Potts models, J. Stat. Phys. 50, 1-40 (1988)

[3] M. Biskup, C. Borgs, J.T. Chayes, R. Kotecký, Phase diagrams of Potts models in external fields, preprint in preparation

[4] M. Biskup, L. Chayes, R. Kotecký, Coexistence of partially disordered/ordered phases in an extended Potts model, preprint

[5] C. Borgs, J.T. Chayes, The covariance matrix of the Potts model: a random cluster analysis, J. Stat. Phys. 82, 1235-1297 (1996)

[6] R.M. Burton, M. Keane, Density and uniqueness in percolation, Commun. Math. Phys. 10, 501-505 (1989) 
[7] J.T. Chayes, A. Puha, T. Sweet, Independent and dependent percolation, in Probability Theory and Applications, IAS/Park City mathematical series, vol. 6, eds. E. P. Hsu, S. R. S. Varadhan, American Mathematical Society and Institute for Advanced Study, 51-118 (1999)

[8] R.G. Edwards, A.D. Sokal, Generalization of the Fortuin-Kasteleyn-Swendsen-Wang representation and Monte Carlo algorithm, Phys. Rev. D 38, 2009-2012 (1988)

[9] A.C.D. van Enter, R. Fernández, A. Sokal, Regularity properties and pathologies of position-space renormalization-group transformations: Scope and limitations of Gibbsian theory, J. Stat. Phys. 72, 8791167 (1993)

[10] C.M. Fortuin, P.W. Kasteleyn, On the random cluster model I. Introduction and relation to other models, Physica 57, 536-564 (1972)

[11] G. Gallavotti, The phase separation line in the two-dimensional Ising model, Commun. Math. Phys. 27, 103 (1972).

[12] H.-O. Georgii, O. Häggström, C. Maes The random geometry of equilibrium phases, preprint.

[13] A. Gandolfi, M. Keane, C. M. Newman Uniqueness of the infinite component in a random graph with applications to percolation and spin glasses, Probab. Theory Relat. Fields 92, 511-527 (1992)

[14] A. Gandolfi, M. Keane, L. Russo, On the uniqueness of the infinite occupied cluster in dependent twodimensional site percolation, Ann. Probab. 16, 1147-1157 (1988)

[15] H.-O. Georgii, Gibbs Measures and Phase Transitions, De Gruyter Studies in Mathematics vol. 9 (De Gruyter, Berlin, 1988)

[16] G.R. Grimmett, The stochastic random cluster process and the uniqueness of random cluster measures, Ann. Prob. 23, 1461-1510 (1995)

[17] O. Häggström, Random cluster representations in the study of phase transitions, Mark. Proc. Rel. Fields 4, 275-321 (1998)

[18] Y. Higuchi, On the absence of non-translationally invariant Gibbs states for the two-dimensional Ising system, In Random Fields: Rigorous Results in Statistical Mechanics and Quantum Field Theory (Esztergom, 1979), J. Fritz, J.L. Lebowitz, D. Szász, eds., Vol. I, 517-534 (North-Holland, Amsterdam, 1981)

[19] R. Kotecký, S.B. Shlosman, First-order transitions in large entropy lattice models, Commun. Math. Phys. 83 493-515 (1982)

[20] C. Preston, Random Fields (Springer, Berlin, 1976)

[21] C.E. Pfister, K. Vande Velde, Almost sure quasilocality in the random cluster model, J. Stat. Phys. 79, 765-774 (1995)

[22] D. Ruelle, Thermodynamic Formalism (Addison-Wesley, London, Amsterdam, Don Mills, Sydney, Tokyo, 1978) 